\title{
HOARD OF ROMAN SILVER COINS FROM THE ROMAN FORUM IN STOBI
}

\begin{abstract}
The Roman silver coins found at the Roman Forum in Stobi (Municipium Stobensium) contained 306 specimens (50 denarii, 255 antoniniani and 1 sestertius). Along with the coins, jewelry was also found (two male rings, a pair of gold earrings and a gold children's earring). The jewelry found along with the collective find of Roman coins, probably represented some kind of "family" savings. According to the content of the hoard, there are 29 emperors or members of their families, from Antoninus Pius to Gallienus. The largest number of money was issued in the period between 238 and $257 / 258$ with a share of $84.7 \%$. The only specimen in Emperor Gallienus's 257/258 empire (from Valerian's joint rule) is an indication of the time of burial.
\end{abstract}

Keywords: Coin hoard, Roman Forum, Stobi, Gallienus, Republic of North Macedonia.

$\mathbf{A}$ rchaeological excavations carried out in 2005 at a site named "Roman Forum" in Stobi (Municipium Stobensium) (see map) also found one hoard with 306 Roman coins. ${ }^{1}$ The hoard was found within the researched Roman public building with arches. ${ }^{2}$ Along with the coins, some jewelry was also discovered (two men's silver rings, a pair of gold earrings and a gold children's earring). ${ }^{3}$ The Forum, the location of the hoard was discovered by excavations in 1972-1974. As a building, it was placed on the eastern terrace of the city, and it was an administrative, commercial, political and cultural-religious center, the site of public gatherings.

\footnotetext{
1 The hoard was found in a rubble dump, which dug up late Roman tombs. The remains of the ground and the shape of the blind coins themselves showed that the hoard and the jewelry were housed in a leather sack. SHURBANOSKA/JAKIMOVSKI 2007: 44-46. The coins were registered with field inventory number M/05/1-306.

2 The existence of the building has been established since excavations in 1972-1974 (САНЕВ/САРЖОВСКИ 1981: 229-234), but intensive excavations after 2003 have revealed in its entirety a significant early Roman construction adorned with luxurious decorative elements. SHURBANOSKA/JAKIMOVSKI 2010: 30-45.

${ }^{3}$ SHURBANOSKA/JAKIMOVSKI 2010: 90.
}

\section{Sanja Bitrak}

Archaeological Museum of Republic of North Macedonia, Skopje e-mail: sanjabitrak@yahoo.com

\section{Pero Josifovski}

Archaeological museum of Republic of North Macedonia, Skopje

e-mail: pjosifovski@yahoo.com

DOI: 10.14795/j.v7i1_SI.476

ISSN 2360 - 266X

ISSN-L 2360 - 266X 


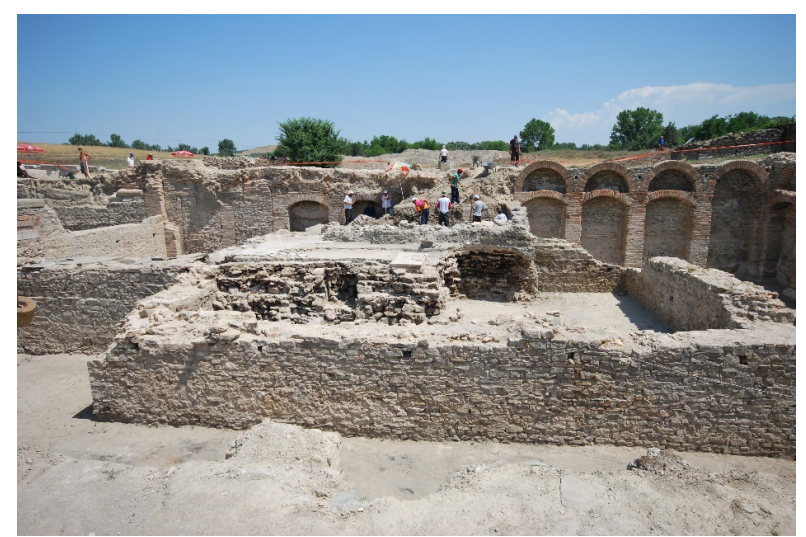

Fig. 1. Roman public building with arches.

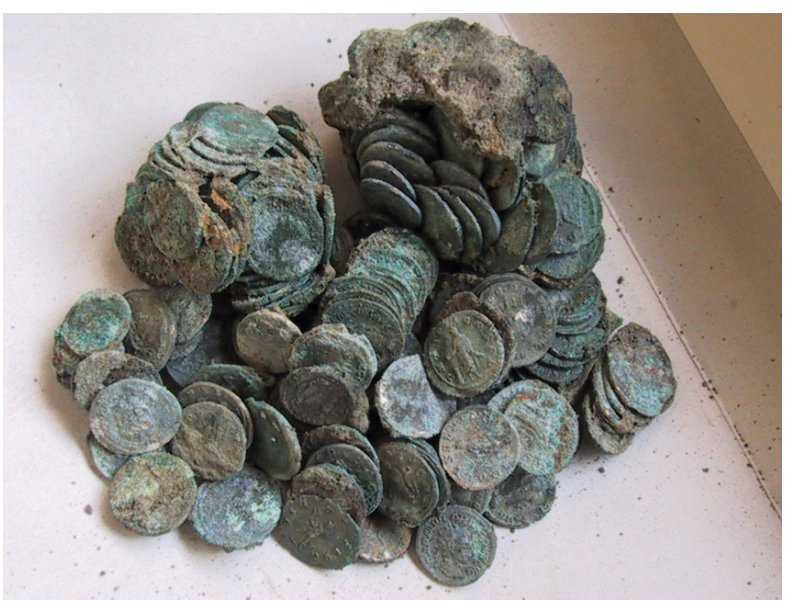

Fig. 2. The hoard before consevation.

The Forum in Stobi was erected at the turn of the 1 st and 2 nd centuries, but actively existed in the 2 nd and 3rd centuries. ${ }^{4}$ Stobi was the largest city in the north of the Province of Macedonia. ${ }^{5}$ With the founding of Roman colonies and the settlement of Roman troops in Macedonia in the late 1st century BC, the town of Stobi acquired a Roman character, a place where a significant community of Roman citizens lived. At the time of August Stobi was oppidum civium Romanorum. Stobi is one of the few cities in this part of the empire that enjoyed Municipium status, ${ }^{6}$ while using ius Italicum. Located on prominent strategic corridors, the Municipium Stobi in the imperial period is a prosperous place and an important economic

\footnotetext{
4 SHURBANOSKA/JAKIMOVSKI 2010: 22.

5 About the history of the city: PAPAZOGLOU 1988: 313-323; JOSIFOVSKI 2001: 20 - 35; МИКУЛЧИК 1999: 160-171.

6 For the transformation of Stobi from oppidum civium Romanorum into Municipium, see PAPAZOGLOU 1986: 213-237.
}

\begin{tabular}{|c|c|c|c|}
\hline Ruler: & Cat. No. & $\begin{array}{l}\text { No. of } \\
\text { coins: }\end{array}$ & \%: \\
\hline Antoninus Pius & Cat. 1 & 1 & 0.32 \\
\hline Septimius Severus & Cat. 26 & 5 & 1.63 \\
\hline Julia Domna & Cat. 7 & 1 & 0.32 \\
\hline Caracalla & Cat. 816 & 9 & 2.93 \\
\hline Plautilla & Cat. 17 & 1 & 0.32 \\
\hline Geta & Cat. 1819 & 2 & 0.65 \\
\hline Elagabalus & Cat. 2025 & 6 & 1.95 \\
\hline Julia Soaemias & Cat. 2627 & 2 & 0.65 \\
\hline Julia Maesa & Cat. 2829 & 2 & 0.65 \\
\hline Severus Alexander & Cat. 3037 & 8 & 2.60 \\
\hline Julia Mamaea & Cat. 3840 & 3 & 0.97 \\
\hline Maximus & Cat. 41 & 1 & 0.32 \\
\hline Maximinus I & Cat. 4246 & 5 & 1.63 \\
\hline Gordian II & Cat. 47 & 1 & 0.32 \\
\hline Balbinus & Cat. 48 & 1 & 0.32 \\
\hline Gordian III & Cat. 49130 & 82 & 26.73 \\
\hline Philip I & Cat. 131172 & 42 & 13.69 \\
\hline Otacilia Severa & Cat. 173182 & 10 & 3.26 \\
\hline Philip II & Cat. 182189 & 7 & 2.28 \\
\hline Trajan Decius & Cat. 190223 & 34 & 11.08 \\
\hline Herennia Etruscilla & Cat. 224226 & 3 & 0.97 \\
\hline Herennius Etruscus & Cat. 227230 & 4 & 1.30 \\
\hline Hostilian & Cat. 231232 & 2 & 0.65 \\
\hline Trebonianus Gallus & Cat. 233269 & 37 & 12.06 \\
\hline Volusian & Cat. 270291 & 22 & 7.17 \\
\hline Aemilian & Cat. 292297 & 6 & 1.95 \\
\hline Valerian & Cat. 298304 & 7 & 2.28 \\
\hline Mariniana & Cat. 305 & 1 & 0.32 \\
\hline Gallienus & Cat. 306 & 1 & 0.32 \\
\hline TOTAL: & & 306 & 100 \\
\hline
\end{tabular}

Fig. 3. Composition of the Stobi Forum hoard in percentage.

center. The city also had its own mint, that for various periods had minted bronze coins for about 150 years (from Emperor Vespasian to Emperor Caracalla). ${ }^{7}$

7 For the activity of the mint, see JOSIFOVSKI 2001: 12ff.; also JOSIFOVSKI 2001a: 55-69; and KUZMANOVIČ COLL.: 13-23. 


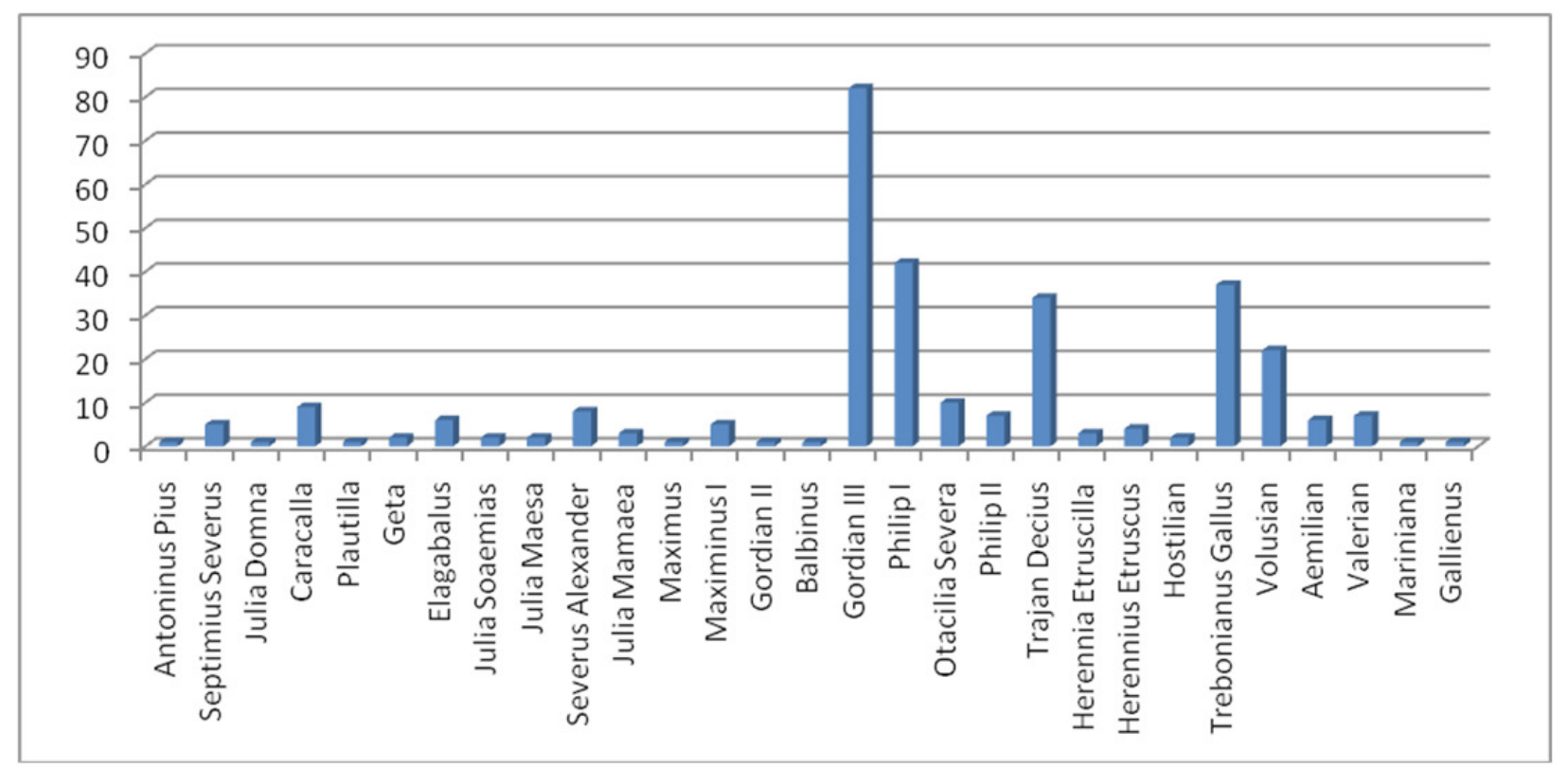

Graph. 1. Composition of the Stobi Forum hoard acording to rulers and family members.

The collective finding contained 306 coins, 1 coin is sestertius, 50 coins are denarii, and 255 are antoniniani. ${ }^{8}$ The found hoard of denarii and antoniniani, with their own coins are represented by 29 emperors or members of their families. This numerous representation reflects the great variety and number of issues. Some of the rulers are represented with only 1 piece each: Antoninus Pius (Cat. No. 1), Julia Domna (Cat. 7), Plautila (Cat. No. 17), Maximus I (Cat. No. 41), Gordian II (Cat. No. 47) ), Balbinus (Cat. 48), Mariniana (Cat. 305), Gallienus (Cat. 306), with two pieces, Geta (Cat. 18-19), Julia Soaemias (Cat. 26-27), Julia Maesa (Cat. 28-29), Hostilian (Cat. 231232); with a few more pieces: Julia Mamaea (3, Cat. 38-40), Herrenia Etruscilla (3, Cat. 224226), Herennius Etruscus (4, Cat. 227-230), Septimius Severus (5, Cat. 2-) 6), Maximinus I (5, Cat. 42-46), Elagabalus (6, Cat. 20-25), Aemilian (6, Cat. 292-297), Philip II (7, Cat. 182-189), Valerian (7, Cat. 298-304), Severus Alexander (8, Cat. 30-37), Caracalla (9, Cat. 8-16), Otacila Severa (10, Cat. 173-182); with more than ten pieces represented: Volusian (22, Cat. 270-291), Trajan Decius (34, Cat.

\footnotetext{
${ }^{8}$ For the purposes of this publication, Forum Romanum Stobis II (2007-2008), an initial general outline and summary of the contents of the hoard is made by Pero Josifovski (JOSIFOVSKI 2010: 82-89).
}

190-223), Trebonianus Gallus (37, Cat. 233269), Philip I (42, Cat. 131-172), and Gordian III (82, Cat. 49-130).

The percentage representation begins with the lowest $0.32 \%$ among the rulers represented by one piece: Antoninus Pius, Julia Domna, Plautila, Maximus, Gordian II, Balbinus, Mariniana and Gallienus; rulers represented by several pieces: Geta, Julia Soaemias, Julia Maesa, Hostilian (all 0.65\%), followed by Julia Mamaea (0.97), Herennia Etruscilla (0.97), Herrenius Etruscus (1.30), Septimius Severus (1.63), Maximinus (1.63), Elagabalus (1.95), Aemilian (1.95), Philip II (2.28), Valerian (2.28), Severus Alexander (2.60); higher percentages of Otacilia Severa (3.26), Volusian (7.17), Trajan Decius (11.08), Trebonianus Gallus (12.06), (13.69), Philip I (13.69), and highest with Emperor Gordian III (26.73\%) more than a quarter of all coins in the hoard. By frequency, the largest number of coins was issued between 238 and 257/58, with $84.7 \%$ (the period of Emperor Gordian II to Emperor Gallienus). It is precisely this period that is the time of the most intense increase in the value of this savings.

Chronologically, the earliest specimen in the hoard is the only bronze coin, a poorly preserved sestertius of the Emperor Antoninus Pius (138-161). If we take away this only one 


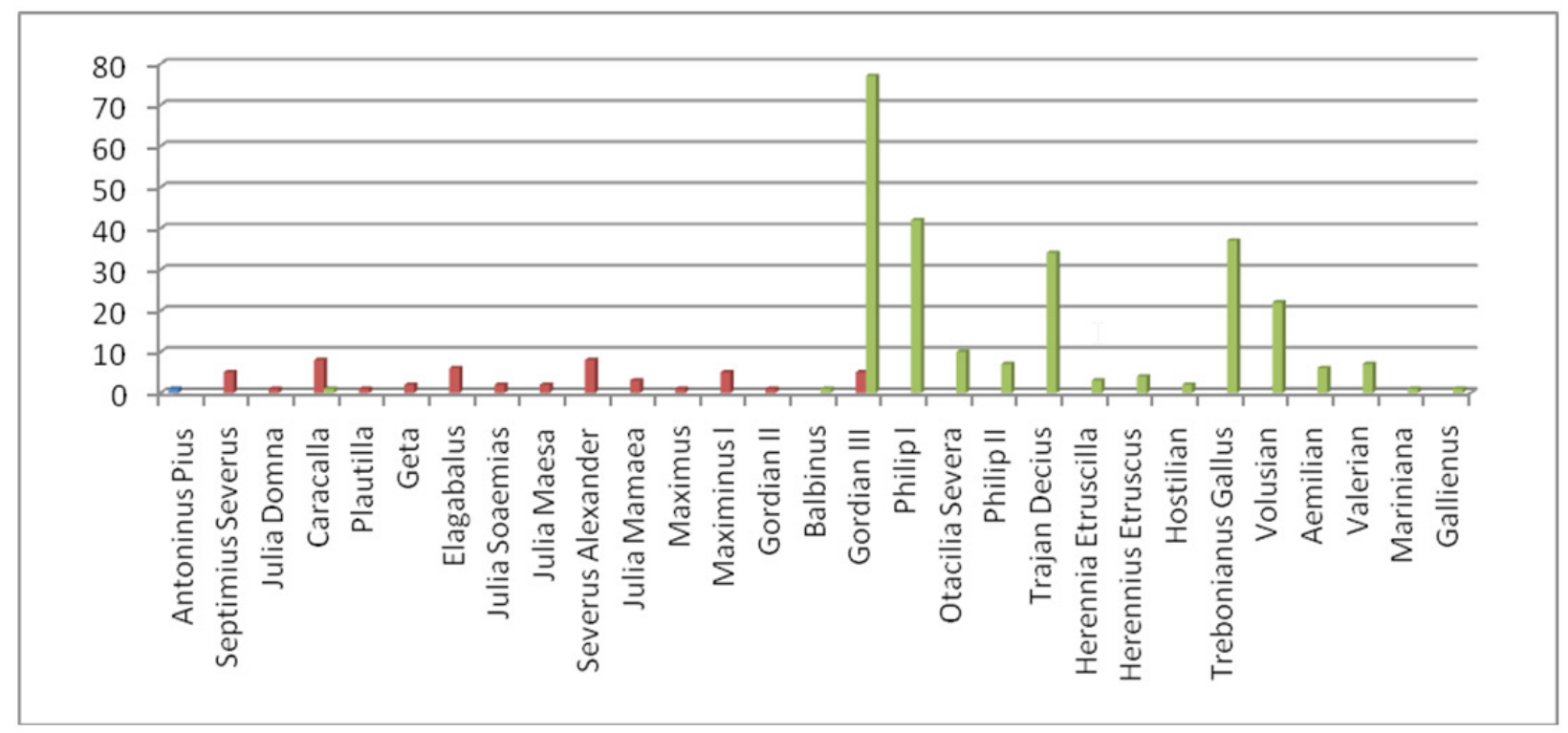

Graph. 2. Presence of denarii and antoninianii acording to rulers and family members.

\begin{tabular}{|c|c|c|c|}
\hline Ruler: & SEST.: & DEN.: & ANT.: \\
\hline Antoninus Pius & 1 & & \\
\hline Septimius Severus & & 5 & \\
\hline Julia Domna & & 1 & \\
\hline Caracalla & & 8 & 1 \\
\hline Plautilla & & 1 & \\
\hline Geta & & 2 & \\
\hline Elagabalus & & 6 & \\
\hline Julia Soaemias & & 2 & \\
\hline Julia Maesa & & 2 & \\
\hline Severus Alexander & & 8 & \\
\hline Julia Mamaea & & 3 & \\
\hline Maximus & & 1 & \\
\hline Maximinus I & & 5 & \\
\hline Gordian II & & 1 & \\
\hline Balbinus & & & 1 \\
\hline Gordian III & & 5 & 77 \\
\hline Philip I & & & 42 \\
\hline Otacilia Severa & & & 10 \\
\hline Philip II & & & 7 \\
\hline Trajan Decius & & & 34 \\
\hline Herennia Etruscilla & & & 3 \\
\hline Herennius Etruscus & & & 4 \\
\hline Hostilian & & & 2 \\
\hline Trebonianus Gallus & & & 37 \\
\hline
\end{tabular}

\begin{tabular}{|c|c|c|c|}
\hline Ruler: & SEST.: & DEN.: & ANT.: \\
\hline Volusian & & & 22 \\
\hline Aemilian & & & 6 \\
\hline Valerian & & & 7 \\
\hline Mariniana & & & 1 \\
\hline Gallienus & & & 1 \\
\hline TOTAL: & $\mathbf{1}$ & 50 & 255 \\
\hline
\end{tabular}

Fig. 4, Presence of denarii and antoninianii acording to rulers and family members.

bronze coin, basically, the creation of this savings began almost four decades later during the reign of Emperor Septimius Severus, with a denarius of it, a Roman issue dated 198200. In this period between $198-200$ and $257-$ 258 all the other 28 rulers are placed, which shows the highest intensity, continuity and systematic accumulation of these monetary values (perhaps in more than one generation). The ending specimen in the hoard is the coin of Emperor Gallienus, the antoninian of the mint in Milan/Mediolanum. ${ }^{9}$ Considering the

9 Other different dates of coinage (254 years) and mint (Viminacium) have been used in the literature for this issue of Galien (other than RIC V / I, Legionary Series, no. 397). For the designation of Viminacium as the coinage place for this issue, dated 254 (issue 3): see VASIĆ 2005: 48, and cat. no. 1585-1591; If one considers such a dating of Gallienus's specimen in 254 then the burial date of Stobi's forum hoard should also 


\begin{tabular}{|c|c|c|c|c|}
\hline Ruler: & Rome & $\begin{array}{c}\text { An- } \\
\text { tioch }\end{array}$ & $\begin{array}{l}\text { Mi- } \\
\text { lan }\end{array}$ & $\begin{array}{l}\text { TO- } \\
\text { TAL: }\end{array}$ \\
\hline Antoninus Pius & 1 & & & 1 \\
\hline Septimius Severus & 5 & & & 5 \\
\hline Julia Domna & 1 & & & 1 \\
\hline Caracalla & 9 & & & 9 \\
\hline Plautilla & 1 & & & 1 \\
\hline Geta & 2 & & & 2 \\
\hline Elagabalus & 6 & & & 6 \\
\hline Julia Soaemias & 2 & & & 2 \\
\hline Julia Maesa & 2 & & & 2 \\
\hline Severus Alexander & 8 & & & 8 \\
\hline Julia Mamaea & 3 & & & 3 \\
\hline Maximus & 1 & & & 1 \\
\hline Maximinus I & 5 & & & 5 \\
\hline Gordian II & 1 & & & 1 \\
\hline Balbinus & 1 & & & 1 \\
\hline Gordian III & 74 & 8 & & 82 \\
\hline Philip I & 39 & 3 & & 42 \\
\hline Otacilia Severa & 10 & & & 10 \\
\hline Philip II & 7 & & & 7 \\
\hline Trajan Decius & 32 & & 2 & 34 \\
\hline Herennia Etruscilla & 3 & & & 3 \\
\hline Herennius Etruscus & 4 & & & 4 \\
\hline Hostilian & 2 & & & 2 \\
\hline Trebonianus Gallus & 22 & 6 & 9 & 37 \\
\hline Volusian & 20 & 1 & 1 & 22 \\
\hline Aemilian & 6 & & & 6 \\
\hline Valerian & 3 & & 4 & 7 \\
\hline Mariniana & 1 & & & 1 \\
\hline Gallienus & 1 & & & 1 \\
\hline TOTAL: & 271 & 18 & 17 & 306 \\
\hline
\end{tabular}

Valerian I indicates the possible time of burial of the hoard in $257 / 258$.

In this hoard of Stobi, dominated by coins with antoninian value (255 pieces) versus those with denarius value (50 pieces), there were three mints in which this money has been coined. These were mints in Roma, Antioch, and Milan. The most common are the coins issued in Roma with high $88.53 \%$ (271 pieces), followed by Antioch's with $5.88 \%$

\begin{tabular}{lcl}
\hline HOARD & $\begin{array}{c}\text { No. of } \\
\text { coins: }\end{array}$ & Buriel date \\
\hline v. Šopur, Štip & 23 & Severus Alexander \\
\hline Unknown location I & 84 & Severus Alexander \\
\hline site "Plaošnik”, Ohrid & 6 & Philip I \\
\hline Unknown location II & 15 & Volusian \\
\hline v. Čepigovo, Prilep & 16 & Valerian I \\
\hline $\begin{array}{l}\text { Stobi (“Forum"), } \\
\text { Gradsko }\end{array}$ & 306 & $\begin{array}{l}\text { Gallienus (join } \\
\text { reign) }\end{array}$ \\
\hline $\begin{array}{l}\text { v. Budur Čiflik, D. } \\
\text { Kapija }\end{array}$ & 16 & Gallienus \\
\hline Kavadarci & 20 & Claudius II \\
\hline $\begin{array}{l}\text { site “Lokubija”, } \\
\text { Kočani }\end{array}$ & 6 & Claudius II \\
\hline $\begin{array}{l}\text { site “Rača", v. } \\
\text { Velestovo, Ohrid }\end{array}$ & $287(?)$ & Florianus(?) \\
\hline \begin{tabular}{l} 
Skopje (1908) \\
\hline
\end{tabular} & $1000+$ & (?) \\
\hline
\end{tabular}

Fig. 6. Hoards from the 3rd century (numer of coins and buriel date).

(18 pieces) and approximately so with Milan $5.55 \%$ (17 pieces).

From the materials published so far, besides the Forum hoard, there are still ten other smaller hoards found on the territory of present-day Republic of North Macedonia (part of former ancient Paionia and Upper Macedonia), whose burial dates back to the 3rd century. ${ }^{10}$ These are hoards from

RIC coin designation, this issue of Gallienus from the period of joint rule with Emperor include the coins of Emperor Valerian (in our cat. No. 301-304). Many of the hoards found in the Balkans from this period ended with specimens of coins of Valerian and Gallienus (joint rule), in particular the concentration of the analogues hoards from Upper Moesia. (BORIĆ BREŠKOVIĆ/STAMENKOVIĆ 2008: 159-164; ВОЈВОДА/СРНДАКОВИЋ 2015: 45-46; АРСЕНИЕВ-ИЋ 2004: 225-234).

10 About the finds of hoards in the wider region, TOURATSOGLOU 1993: table "Silver Coin Hoards from Macedonia (ca. 200 B.C. - 3rd cent. A.D.); TOURATSOGLOU 2006: 152-157, 196-201 (Coin Hoards of the 3rd century AD from Greece and the Balkans). 


\begin{tabular}{|l|c|c|c|c|c|}
\hline HOARD & DEN. & ANT. & SEST. & AS & E \\
\hline v. Šopur, Štip & 23 & & & & \\
\hline Unknown location I & 84 & & & & \\
\hline site “Plaošnik”, Ohrid & & & 5 & 1 & \\
\hline Unknown location II & & & & & 15 \\
\hline v. Čepigovo, Prilep & 2 & 14 & & & \\
\hline Stobi (“Forum”), Gradsko & 50 & 255 & 1 & & \\
\hline v. Budur Čiflik, D. Kapija & 15 & 1 & & & \\
\hline Kavadarci & & 1 & 2 & & 17 \\
\hline site “Lokumrija”, Kočani & & 6 & & & \\
\hline site "Rača”, v. Velestovo, Ohrid ${ }^{1}$ & $30(?)$ & $257(?)$ & & & \\
\hline Skopje & $(?)$ & $(?)$ & & & \\
\hline Skopje (1908) & $746(?)$ & $274(?)$ & & & \\
\hline TOTAL: & $\mathbf{9 4 8}$ & $\mathbf{8 0 8}$ & $\mathbf{8}$ & $\mathbf{1}$ & $\mathbf{3 2}$ \\
\hline
\end{tabular}

Fig. 7. Hoards from the 3rd century (Presence of denarii, antoninianii and bronze coins).

Šopur, ${ }^{11}$ Plaošnik, ${ }^{12}$ Čepigovo, ${ }^{13}$ Budur Čiflik, ${ }^{14}$ Kavadarci, ${ }^{15}$ Kočani, ${ }^{16}$ Velestovo, ${ }^{17}$ Skopje, ${ }^{18}$ Skopje (1908), ${ }^{19}$ and two left with an unknown place of discovery. ${ }^{21}$ As we can see from the figure 6 , it is most often hoards with fewer coins, except the ones from Forum Stobi,

\footnotetext{
${ }^{11}$ KERAMIDČIEV 1972: 71; KERAMIDČIEV 1973: 25 28; MIRNIK 1981: 72, No. 236.

12 BITRAK 2016: 76-77.

13 KEPESKI 1992: 183-190.

${ }^{14}$ KERAMIDČIEV 1973: 31-33; MIRNIK 1981: 61, No. 158.

15 JOSIFOVSKI 2010: 21.

${ }^{16}$ ATAHACOBA 2010: 271-274.

17 КЕРАМИДЧИЕВ/АЛЕКСОВА 1966: 13; КЕRАMIDČIEV 1972: 71; MIRNIK 1981: 73, No. 242; РАЗМОВСКА БАЧЕВСКА 2002: 32-33.

18 MIRNIK 1981: 70, No. 220; VASIĆ 1972: 60.

19 KUBITSCHEK 1908: 37-54; MIRNIK 1981: 70, No. 219.
}

${ }^{20}$ According to Keramidchiev, the hoard numbered 298, (КЕРАМИДЧИЕВ/АЛЕКСОВА 1966: 13); to Mirnik that hoard number 298 specimens (MIRNIK 1981: 73, No. 242); whereas according to Razmovska Bačevska there are 322 coins (?), but according to the list of the rulers represented there, the sum of the coins is 287 pieces! (РАЗМОВСКА БАЧЕВСКА 2002: 32-33). А new definition of the number and content of the hoard is needed.

${ }^{21}$ OHRIDSKA BANKA COLL. 2007: 253 (Hoard from unknown location I, Roman denarii), and 253-254 (Hoard from unknown location II, bronze coins from Viminacium).
Budur Čiflik, and the dispersed find from Skopje (1908) which count slightly more, all others being with much smaller number of coins, with less than a hundred pieces.

For the most part, the contents of the hoards consist of denarii and antoniniani, and only the hoards of Kavadarci and one of the unknown locations are dominated by bronze coins (bronze coins of Stobi on one side and bronze coins of Viminacium on the other). According to the chronology of burying the hoards, two hoards date back to Severus Alexander, one to Philip I, Volusian and Valerian I, two to Gallienus and Claudius II. At the same time as the burial with the Stobi hoards there is only the Budur Čivlik, Demir Kapija. The geographical distribution of the hoards from the third century AD on the territory of today's Republic of North Macedonia shows their distribution on the main road corridors, namely: the road corridor along the Vardar River (Axios) in the direction Thessaloniki - Singidunum, the road directions leading from Stobi to Lychnidos and the other from Stobi along the river Bregalnica towards Pautalia and Serdica.

The first half of the third century, the period during which the "family" savings were created by the Roman Forum in Stobi, is a 
period in which in Macedonia circulation and daily exchange are used bronze coins issued by local mints, parallel to those of the central mint. ${ }^{22}$ Valuable currencies, such as denarii and antoninani, which are also in circulation, are also used in savings accumulation processes, and as the contents of discovered hoards show, they are more preferred than local bronze money in those processes.

As to the possible reasons for the burial of our "family" wealth of Roman money and jewelry we can only guess. The middle of the third century is a tumultuous period in the Roman Empire, a period of military-political crisis, unrest, military strife, and especially barbarian invasions, who did not cross Macedonia. ${ }^{23}$ The turbulent times of previous years continued in the period from 254 to 260 , and are also referred to by the numerous buried hoards of money and jewelry found throughout the Balkans (Pannonia, Upper and Lower Moesia, Thrace, Dacia, Macedonia ${ }^{24}$ ). The fact that the hoard from the Forum in Stobi was found in a layer of debris buried in late Roman tombs indicates that the hoard was buried at some necessary and uncertain times.

*Author of the photos Pero Josifovski and Sanja Bitrak, except fig. 1-2, Mila Shurbanoska

\footnotetext{
22 For possible models of monetary circulation in Roman Macedonia see: KREMYDI-SICILIANOU 2004: 135-149; TOURATSOGLOU 2006: 153-154; TOURATSOGLOU 1993: 41.

${ }^{23}$ TOURATSOGLOU 2006: 144-148.

24 TOURATSOGLOU 2006: 135-136, 156.
}

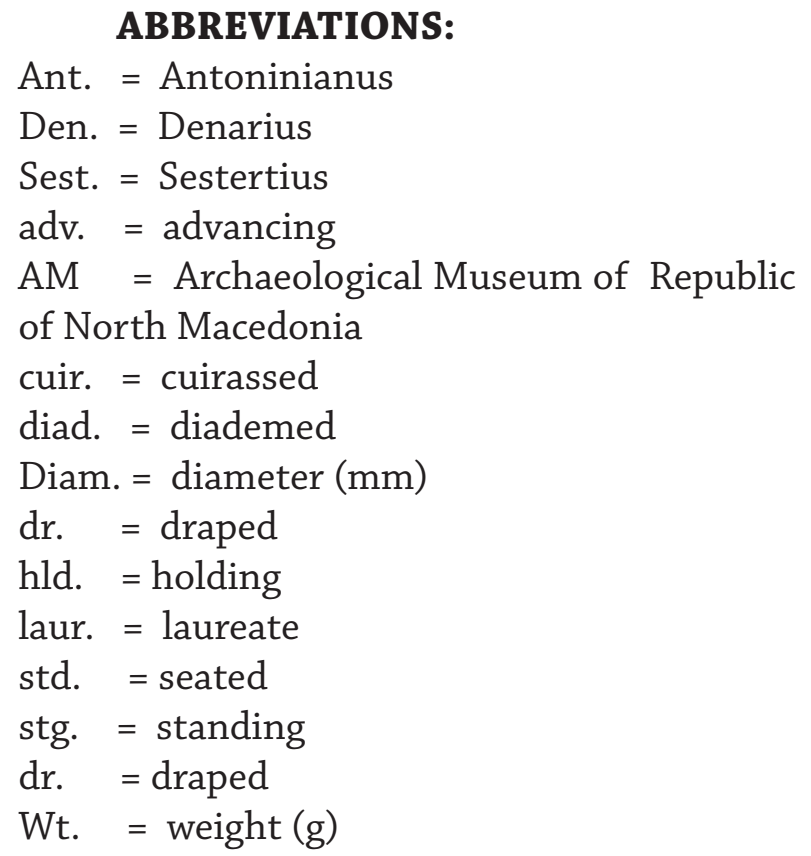




\section{CA T A L O G U E}

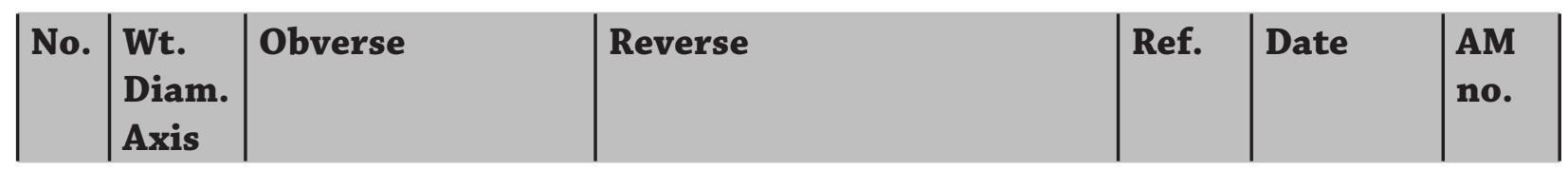

\section{ANTONIUS PIUS \\ (cat. 1)}

\section{Mint of Rome (?)}

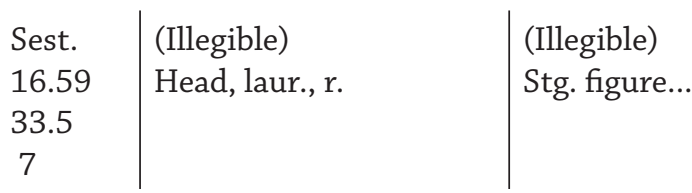

(Illegible)

Stg. figure...

\section{SEPTIMIUS SEVERUS}

(cat. 2-6)

\section{Mint of Rome}

\begin{tabular}{|l|l|l|}
2 & DEN. & L [SEPT] SEV AVG IMP \\
& 2.92 & XI PART MAX \\
& 17.9 & Head, laur., r. \\
& 7 & \\
& & \\
& & \\
& DEN. & SEVERVS AVG \\
& 18.8 & PART MAX \\
& 5 & Head, laur., r. \\
& & \\
& DEN. & SEVERVS PIVS AVG \\
& 2.97 & Head, laur., r. \\
& 20.1 & \\
& 7 & \\
& & \\
5 & DEN. & SEVERVS PIVS AVG \\
& 2.57 & As previous. \\
& 19.0 & \\
& 7 & \\
& & \\
6 & DEN. & SEVERVS PIVS AVG \\
& 2.74 & BRIT \\
& 18.9 & As previous. \\
& 1 &
\end{tabular}

MARTI VI-CTORI

Mars, helmeted, naked to waist, chlamys dr. over l. arm, stg half r., hld. spear, $r$. hand on shield resting on helmet.

\section{RESTITVTOR VRBIS}

Severus stg. 1., sacrificing with

patera over tripod and hld. spear.

VOTA SVS-CEPTA XX

Severus, stg. l., sacrificing

from patera over altar.

VOTA SVS-CEPTA XX

As previous.

VICTORIAE B-RIT

Victory std. 1. on shield, with shield on her knee and palm.

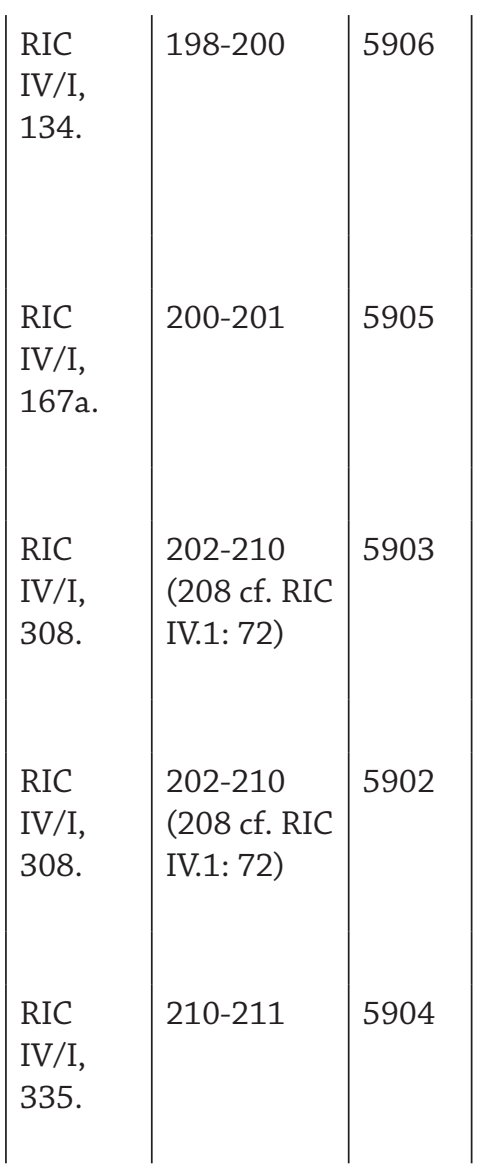




\title{
JULIA DOMNA \\ (Striking under Septimius Severus) \\ (cat. 7) \\ Mint of Rome
}

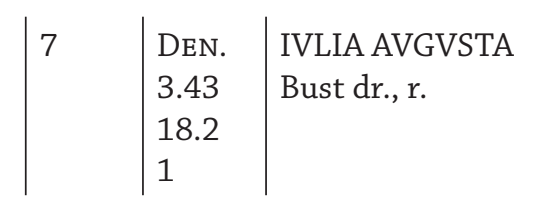

\author{
SAECVLI FELICITAS \\ Horus; behind rudder.

\section{CARACALLA \\ (cat. 8-16) \\ Mint of Rome}

RIC $\mid 196-211$

6128

Isis, stg. r., l. foot on prow, hld.

(ca. 200)

IV/I

577.

\begin{tabular}{|c|c|c|c|c|c|c|}
\hline 8 & $\begin{array}{l}\text { DEN. } \\
2.97 \\
20.4 \\
11\end{array}$ & $\begin{array}{l}\text { ANTONINVS } \\
\text { AVGVSTVS } \\
\text { Bust, laur., dr., cuir. r. }\end{array}$ & $\begin{array}{l}\text { PONTIF TR P III } \\
\text { Sol nude, stg. front, head l., hld. } \\
\text { globe } \\
\text { and transverse spear. }\end{array}$ & $\begin{array}{l}\text { RIC } \\
\text { IV/I, } \\
\text { 30a. }\end{array}$ & 200 & 5916 \\
\hline 9 & $\begin{array}{l}\text { DEN. } \\
3.17 \\
19.5 \\
6\end{array}$ & $\begin{array}{l}\text { ANTONINVS PIVS } \\
\text { AVG } \\
\text { Bust, laur., r. }\end{array}$ & $\begin{array}{l}\text { PONTIF TR P X COS II } \\
\text { Mars with cloak flying, adv. r., hld. } \\
\text { spear and trophy. }\end{array}$ & $\begin{array}{l}\text { RIC } \\
\text { IV/I, } \\
88 .\end{array}$ & 207 & 5912 \\
\hline 10 & $\begin{array}{l}\text { DEN. } \\
2.91 \\
19.6 \\
1\end{array}$ & $\begin{array}{l}\text { ANTONINVS PIVS } \\
\text { AVG } \\
\text { As previous. }\end{array}$ & $\begin{array}{l}\text { PONTIF TR P XII COS III } \\
\text { Concordia std. l., hld. patera and } \\
\text { double cornucopiae }\end{array}$ & $\begin{array}{l}\text { RIC } \\
\text { IV/I, } \\
111 .\end{array}$ & 209 & 5909 \\
\hline 11 & $\begin{array}{l}\text { DEN. } \\
2.38 \\
19.8 \\
7\end{array}$ & $\begin{array}{l}\text { ANTONINVS PIVS } \\
\text { AVG } \\
\text { As previous. }\end{array}$ & $\begin{array}{l}\text { LIBERALI-TAS AVG VI } \\
\text { Liberalitas stg. l., hld. abacus and } \\
\text { cornucopiae. }\end{array}$ & $\begin{array}{l}\text { RIC } \\
\text { IV/I, } \\
158\end{array}$ & 206-210 & 5910 \\
\hline 12 & $\begin{array}{l}\text { DEN. } \\
3.24 \\
19.4 \\
7\end{array}$ & $\begin{array}{l}\text { ANTONINVS PIVS } \\
\text { AVG } \\
\text { As previous. }\end{array}$ & $\begin{array}{l}\text { SECVRIT IMPERII } \\
\text { Securitas std. r., on chair with } \\
\text { cornucopiae as arms, propping head } \\
\text { on r. and hld. sceptre. In front, altar. }\end{array}$ & $\begin{array}{l}\text { RIC } \\
\text { IV/I, } \\
168 .\end{array}$ & 206-210 & 5915 \\
\hline 13 & $\begin{array}{l}\text { DEN. } \\
2.79 \\
18.4 \\
11\end{array}$ & $\begin{array}{l}\text { ANTONINVS PIVS } \\
\text { AVG BRIT } \\
\text { As previous. }\end{array}$ & $\begin{array}{l}\text { FORT RED PM TR P XIIII COS III } \\
\text { PP } \\
\text { Fortuna stg. l., hld. cornucopiae and } \\
\text { leaning l. on inverted rudder; at her } \\
\text { feet, l., wheel. }\end{array}$ & $\begin{array}{l}\text { RIC } \\
\text { IV/I, } \\
189 .\end{array}$ & 211 & 5911 \\
\hline 14 & $\begin{array}{l}\text { DEN. } \\
3.28 \\
19.2 \\
7\end{array}$ & $\begin{array}{l}\text { ANTONINVS PIVS } \\
\text { AVG BRIT } \\
\text { As previous. }\end{array}$ & $\begin{array}{l}\text { MARTI PROBV-GNATORI } \\
\text { Mars hurrying l., hld. spear and } \\
\text { trophy. }\end{array}$ & $\begin{array}{l}\text { RIC } \\
\text { IV/I, } \\
223\end{array}$ & $210-213$ & 5914 \\
\hline
\end{tabular}




\begin{tabular}{|l|l|l}
15 & ANT. & ANTONINVS PIVS \\
& 5.22 & AVG GERM \\
& 23.3 & $\begin{array}{l}\text { Bust, radiate, } \\
\text { cuirassed, } r .\end{array}$ \\
& 7 & \\
16 & DEN. & ANTONINVS PIVS \\
& 2.93 & AVG GERM \\
& 21.2 & Head, laur., r. \\
& 7 &
\end{tabular}

P M TR P XVIII COS IIII P P
Pluto, with polos on head std. l.,
scepter in l. hand, r. hand extended
towards Cerberus, l., before him.
P M TR P XVIIII COS IIII P P
Jupiter naked, stg. front, head l.,
hld.
thunderbolt and scepter.

\begin{tabular}{|l|l|l|} 
RIC & 215 & 5907 \\
IV/I, & & \\
261c. & & \\
& & \\
RIC & 216 & 5908 \\
IV/I, & & \\
275a. & &
\end{tabular}

PLAUTILLA

(Striking under Caracalla)

(cat. 17)

\section{Mint of Rome}

\begin{tabular}{|l|l|l}
17 & Den. & PLAVTILLAE \\
& 3.02 & AVGVSTAE \\
& 19.8 & Bust, dr., r. \\
& 5 &
\end{tabular}

CONCORDIA AETERNAE
Caracalla stg. l., clasping r. hands
with Plautilla, stg. r.

\begin{tabular}{|l|l|l} 
RIC & $202 \mathrm{ff}$. & 5924 \\
IV/I, & & \\
361. &
\end{tabular}

\section{GETA}

(cat. 18-19)

\section{Mint of Rome}

\begin{tabular}{|c|c|c|}
\hline 18 & $\begin{array}{l}\text { DEN. } \\
3.22 \\
20.0 \\
6\end{array}$ & $\begin{array}{l}\text { P SEPTIMIVS GETA } \\
\text { CA[ES] } \\
\text { Bust, dr., cuir., head } \\
\text { bare, } r \text {. }\end{array}$ \\
\hline 19 & $\begin{array}{l}\text { DEN. } \\
3.19 \\
19.5 \\
6\end{array}$ & $\begin{array}{l}\text { GETA CAES PONT COS } \\
\text { As previous. }\end{array}$ \\
\hline
\end{tabular}

PONTIF COS
Minerva stg. l., leaning on shiled
and
hld. spear.
VOTA PVBLICA
Geta stg. l., sacrificing out of patera
over tripod and hld. scroll.

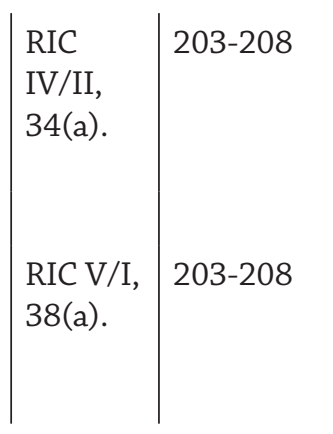

5918

ELAGABALUS

(cat. 20-25)

\section{Mint of Rome}

\begin{tabular}{|l|l|l|}
20 & DEN. & IMP ANTONINVS PIVS \\
& 3.28 & AVG \\
& 19.3 & Bust dr., cuir., head laur., r. \\
& 1 & \\
& & \\
& & \\
& DEN. & IMP ANTONINVS PIVS \\
& 2.13 & AVG \\
& 78.9 & As previous. \\
& DEN. & IMP ANTONINVS PIVS \\
& 2.94 & AVG \\
& 20.5 & Bust dr., head laur., \\
& 6 & horned, r. \\
& &
\end{tabular}

P M TR P III COS III PP

Sol rad., adv. 1., raising $r$, hand and

hld. whip; in 1. field, star.

P M TR P III COS III PP

As previous, but without star.

SACERD DEI ELAGAB

Elagalbalus stg. r., sacrificising over

lighted altar, in field r., star.

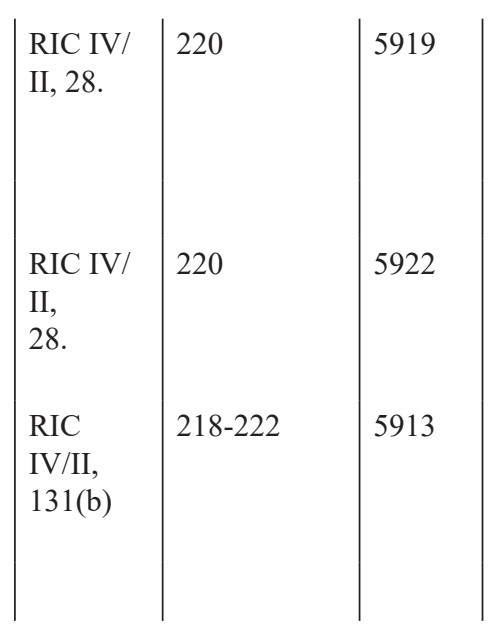




\begin{tabular}{|l|l|l}
23 & DEN. & IMP ANTONINVS PIVS \\
& 2.64 & AVG \\
& 19.8 & Bust dr., head laur., r. \\
& 1 & \\
& & \\
& & DEN. \\
& 2.71 & IMP ANTONINVS AVG \\
& 19.2 & \\
& 11 & \\
& & \\
& Den previous. \\
& 3.30 & IMP CAES M AVR \\
& 18.4 & ANTONINVS AVG \\
& 7 &
\end{tabular}

LIBERTAS AVG
Libertas stg. 1., hld. pileus and

scepter, in filed r., star.

TEMPORVM FELICITAS

Felicitas stg. 1., hld. caduceus and cornucopiae.

VICTOR ANTONINI AVG

Victory running r., hld. wreath and palm.

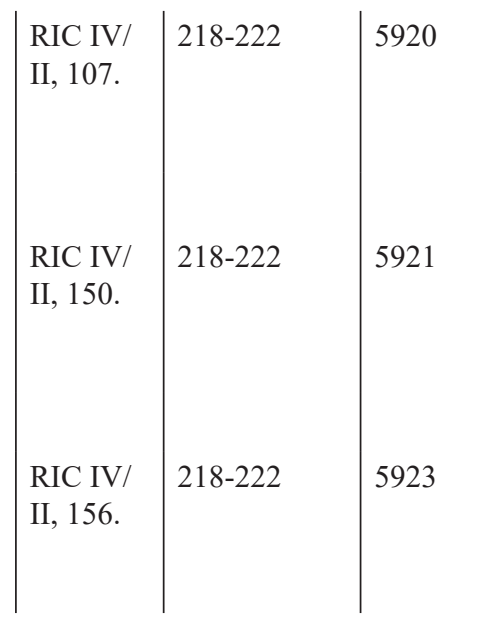

JULIA SOAEMIAS

(Striking under Elagabalus)

(cat. 26-27)

\section{Mint of Rome}

\begin{tabular}{|l|l|l|}
26 & DEN. & IVLIA SOAEMIAS AVG \\
& 2.44 & Bust dr., r. \\
& 19.4 & \\
& 7 & \\
27 & & \\
& DEN. & IVLIA SOAEMIAS AVG \\
& 1.90 & As previous. \\
& 19.5 & \\
& 7 &
\end{tabular}

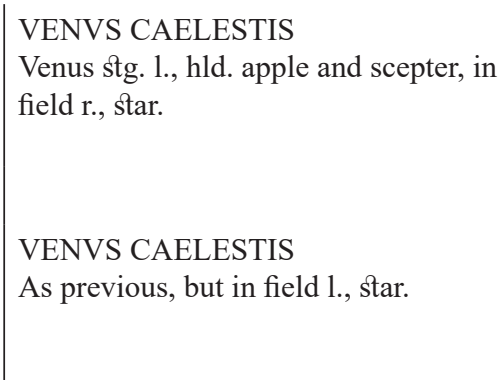

JULIA MAESA

(Striking under Elagabalus)

(cat. 28-29)

\section{Mint of Rome}

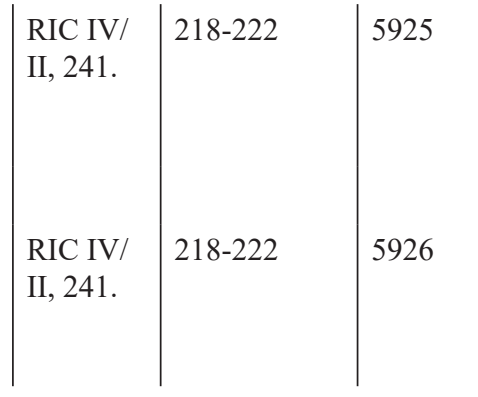

\begin{tabular}{|l|l|l}
28 & DEN. & IVLIA MAESA AVG \\
& 2.37 & Bust diad., $\mathrm{r}$. \\
& 20.3 & \\
& 6 & \\
29 & & \\
& & \\
& DEN. & IVLIA MAESA AVG \\
& 18.95 & As previous. \\
& 7 &
\end{tabular}

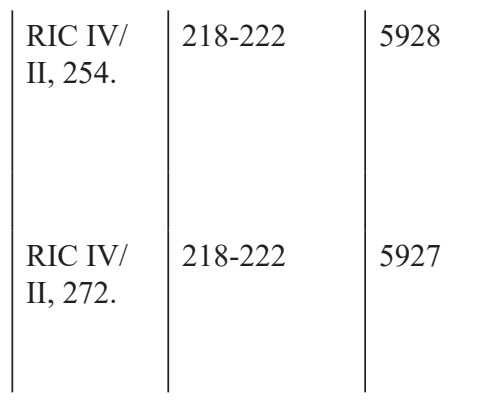

\section{SEVERUS ALEXANDER}

(cat. 30-37)

\section{Mint of Rome}

\begin{tabular}{|l|l}
30 & DEN. \\
& 2.70 \\
& 18.2 \\
7
\end{tabular}

IMP C M AVR SEV ALEXAND AVG

Bust, dr., head laur., r.

PM TR P IIII COS P P

Emperor in military dress stg. 1., hld.

globe and spear reversed. 


\begin{tabular}{|c|c|c|c|c|c|c|}
\hline 31 & $\begin{array}{l}\text { DEN. } \\
2.65 \\
19.6 \\
12\end{array}$ & $\begin{array}{l}\text { IMP SEV ALE-XAND } \\
\text { AVG } \\
\text { Head, laur., r. }\end{array}$ & $\begin{array}{l}\text { PM TR P VII C-OS II P P } \\
\text { Mars walking r., carrying spear and } \\
\text { trophy. }\end{array}$ & $\begin{array}{l}\text { RIC IV/ } \\
\text { II, } 73 .\end{array}$ & 228 & 5934 \\
\hline 32 & $\begin{array}{l}\text { DEN. } \\
2.95 \\
19.8 \\
11\end{array}$ & $\begin{array}{l}\text { IMP SEV ALE-XAND } \\
\text { AVG } \\
\text { As previous. }\end{array}$ & $\begin{array}{l}\text { PM TR P VII COS II P P } \\
\text { Mars stg. r. hld. spear and resting r. hand on } \\
\text { shiled. }\end{array}$ & $\begin{array}{l}\text { RIC IV/ } \\
\text { II, } 74 .\end{array}$ & 228 & 5935 \\
\hline 33 & $\begin{array}{l}\text { DEN. } \\
3.48 \\
18.4 \\
7\end{array}$ & $\begin{array}{l}\text { IMP ALEXAN-DER } \\
\text { PIVS AVG } \\
\text { Bust, laur., dr., cuir., r. }\end{array}$ & $\begin{array}{l}\text { P M TR P X COS III P P } \\
\text { Sol rad., stg. 1., raised r. hand and } \\
\text { hld. globe in } 1 . \text { hand. }\end{array}$ & $\begin{array}{l}\text { RIC IV/ } \\
\text { II, } 109 .\end{array}$ & 231 & 5930 \\
\hline 34 & $\begin{array}{l}\text { DEN. } \\
3.03 \\
18.8 \\
7\end{array}$ & $\begin{array}{l}\text { IMP C M AVR SEV } \\
\text { ALEXAND AVG } \\
\text { Bust, laur., dr., r. }\end{array}$ & $\begin{array}{l}\text { ANNONA AVG } \\
\text { Annona stg. 1., hld. corn-ears and } \\
\text { cornucopiae; at her feet 1., modius. }\end{array}$ & $\begin{array}{l}\text { RIC IV/ } \\
\text { II, } 133 .\end{array}$ & $222-228$ & 5936 \\
\hline 35 & $\begin{array}{l}\text { DEN. } \\
2.31 \\
19.7 \\
7\end{array}$ & $\begin{array}{l}\text { IMP SEV ALE-XAND } \\
\text { AVG } \\
\text { Head, laur., r., slight dr. } \\
\text { on 1. shoulder. }\end{array}$ & $\begin{array}{l}\text { ANNO-NA AVG } \\
\text { Annona stg. 1., hld. corn-ears and } \\
\text { anchor; at her feet, modius (?). }\end{array}$ & $\begin{array}{l}\text { RIC IV/ } \\
\text { II, } 188 .\end{array}$ & $228-231$ & 5929 \\
\hline 36 & $\begin{array}{l}\text { DEN. } \\
3.00 \\
21.4 \\
7\end{array}$ & $\begin{array}{l}\text { IMP ALEXANDER PIVS } \\
\text { AVG } \\
\text { Bust, laur., dr., cuir., r. }\end{array}$ & $\begin{array}{l}\text { MARS V-LTOR } \\
\text { Mars walking r., hld. spear and } \\
\text { shiled. }\end{array}$ & $\begin{array}{l}\text { RIC IV/ } \\
\text { II, } 246 .\end{array}$ & $231-235$ & 5932 \\
\hline 37 & $\begin{array}{l}\text { DEN. } \\
3.08 \\
20.4 \\
1\end{array}$ & $\begin{array}{l}\text { IMP ALEXANDER PIVS } \\
\text { AVG } \\
\text { Head, laur., slight dr. } \\
\text { on l. shoulder, r. }\end{array}$ & $\begin{array}{l}\text { PROVIDENTIA AVG } \\
\text { Providentia stg. front, head turned 1., } \\
\text { hld. corn-ears and anchor; on ground, } \\
\text { modius. }\end{array}$ & $\begin{array}{l}\text { RIC IV/ } \\
\text { II, } 252 .\end{array}$ & $231-235$ & 5931 \\
\hline
\end{tabular}

\section{JULIA MAMAEA \\ (Striking under Alexander Severus) \\ (cat.38-40) \\ Mint of Rome}

\begin{tabular}{|l|l|l}
38 & DEN. & IVLIA MAMAEA AVG \\
& 2.56 & Bust, dr., r. \\
& 20.2 & \\
& 7 & \\
& & \\
& Den. & IVLIA MA-MAEA AVG \\
& 2.94 & Bust diad., dr., r. \\
& 7 & \\
& & \\
40 & Den. & \\
& 3.38 & IVLIA MA-MAEA AVG \\
& 20.9 & \\
& 1 &
\end{tabular}

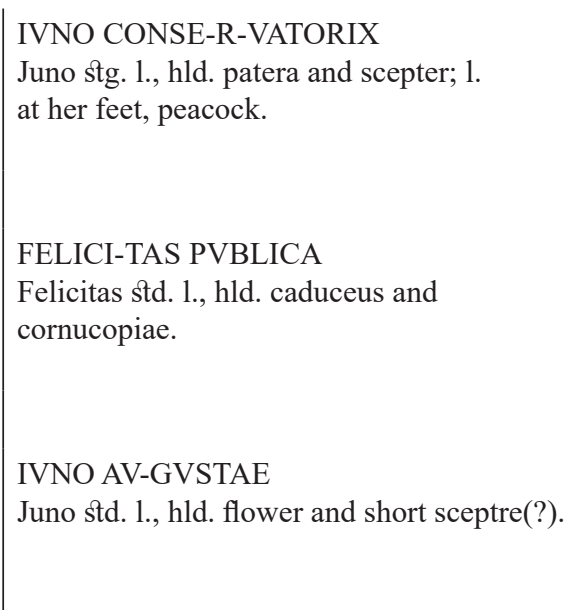

\begin{tabular}{|l} 
RIC IV/ \\
II, 343. \\
RIC IV/ \\
II, 338. \\
\\
\\
RIC IV/ \\
III, \\
341.
\end{tabular}

5937

Juno stg. 1., hld. patera and scepter; 1 .

Mars walking r., carrying spear and

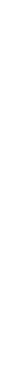




\section{Mint of Rome}

41

DEN.

3.46

20.9

12

IVL VERVS

MAXIMVS CAES

Bust r., bare headed, dr.

Jug between lituus and knife to 1 .,

simpulum and sprinkler.

\section{MAXIMINUS I}

(cat. 42-46)

\section{Mint of Rome}

\begin{tabular}{|c|c|c|}
\hline 42 & $\begin{array}{l}\text { DEN. } \\
2.15 \\
22.2 \\
7\end{array}$ & $\begin{array}{l}\text { IMP MAXIMINVS PIVS } \\
\text { AVG } \\
\text { Bust r., laur., dr., cuirassed. }\end{array}$ \\
\hline 43 & $\begin{array}{l}\text { DEN. } \\
2.89 \\
20.0 \\
6\end{array}$ & $\begin{array}{l}\text { IMP MAXIMINVS } \\
\text { PIVS AVG } \\
\text { As previous. }\end{array}$ \\
\hline 44 & $\begin{array}{l}\text { DEN. } \\
3.14 \\
20.7 \\
7\end{array}$ & $\begin{array}{l}\text { IMP MAXIMINVS } \\
\text { PIVS AVG } \\
\text { As previous. }\end{array}$ \\
\hline 45 & $\begin{array}{l}\text { DEN. } \\
3.12 \\
20.3 \\
1\end{array}$ & $\begin{array}{l}\text { IMP MAXIMINVS } \\
\text { PIVS AVG } \\
\text { As previous. }\end{array}$ \\
\hline 46 & $\begin{array}{l}\text { Den. } \\
2.96 \\
19.1 \\
1\end{array}$ & $\begin{array}{l}\text { MAXIMINVS PIVS } \\
\text { AVG GERM } \\
\text { Bust laur., dr., cuir. r. }\end{array}$ \\
\hline
\end{tabular}

P M TR P P P

Emperor in military dress, stg. 1 .

between two standards, leaning 1., on

spear and raising $r$

\section{FIDES MILITVM}

Fides stg. facing, head 1., hld.

military standard in each hand.

Pax stg 1., hld. branch and transverse scepter.

\section{VICTO-RIA AVG}

Victory advancing r., hld. wreath and palm.

\section{PROVIDENTIA AVG}

Providentia stg. 1., hld. wand over globe and cornucopiae.

\section{PAX AVGVSTI}

RIC IV

II, 1.

5940 


\section{Mint of Rome}

\begin{tabular}{|c|c|c|}
\hline 49 & $\begin{array}{l}\text { ANT. } \\
4.57 \\
21.8 \\
7\end{array}$ & $\begin{array}{l}\text { IMP CAES M ANT } \\
\text { GORDIANVS AVG } \\
\text { As previous. }\end{array}$ \\
\hline 50 & $\begin{array}{l}\text { AnT. } \\
4.76 \\
22.2 \\
1\end{array}$ & $\begin{array}{l}\text { IMP CAES M ANT } \\
\text { GORDIANVS AVG } \\
\text { As previous. }\end{array}$ \\
\hline 51 & $\begin{array}{l}\text { AnT. } \\
4.43 \\
21.8 \\
1\end{array}$ & $\begin{array}{l}\text { IMP CAES M ANT } \\
\text { GORDIANVS AVG } \\
\text { As previous. }\end{array}$ \\
\hline 52 & $\begin{array}{l}\text { AnT. } \\
5.60 \\
22.4 \\
1\end{array}$ & $\begin{array}{l}\text { IMP CAES M ANT } \\
\text { GORDIANVS AVG } \\
\text { As previous. }\end{array}$ \\
\hline 53 & $\begin{array}{l}\text { ANT. } \\
4.00 \\
23.7 \\
1\end{array}$ & $\begin{array}{l}\text { IMP CAES M ANT } \\
\text { GORDIANVS AVG } \\
\text { As previous. }\end{array}$ \\
\hline 54 & $\begin{array}{l}\text { ANT. } \\
4.47 \\
23.9 \\
11\end{array}$ & $\begin{array}{l}\text { IMP CAES M ANT } \\
\text { GORDIANVS AVG } \\
\text { As previous. }\end{array}$ \\
\hline 55 & $\begin{array}{l}\text { AnT. } \\
3.40 \\
23.3 \\
11\end{array}$ & $\begin{array}{l}\text { IMP C M ANT } \\
\text { GORDIANVS AVG } \\
\text { As previous. }\end{array}$ \\
\hline 56 & $\begin{array}{l}\text { ANT. } \\
4.55 \\
21.9 \\
7\end{array}$ & $\begin{array}{l}\text { IMP CAES M ANT } \\
\text { GORDIANVS AVG } \\
\text { As previous. }\end{array}$ \\
\hline 57 & $\begin{array}{l}\text { ANT. } \\
5.07 \\
23.6 \\
7\end{array}$ & $\begin{array}{l}\text { IMP CAES M ANT } \\
\text { CORDIANVS AVG } \\
\text { As previous. }\end{array}$ \\
\hline 58 & $\begin{array}{l}\text { ANT. } \\
4.52 \\
23.8 \\
12\end{array}$ & $\begin{array}{l}\text { IMP CAES M ANT } \\
\text { GORDIANVS AVG } \\
\text { As previous. }\end{array}$ \\
\hline
\end{tabular}

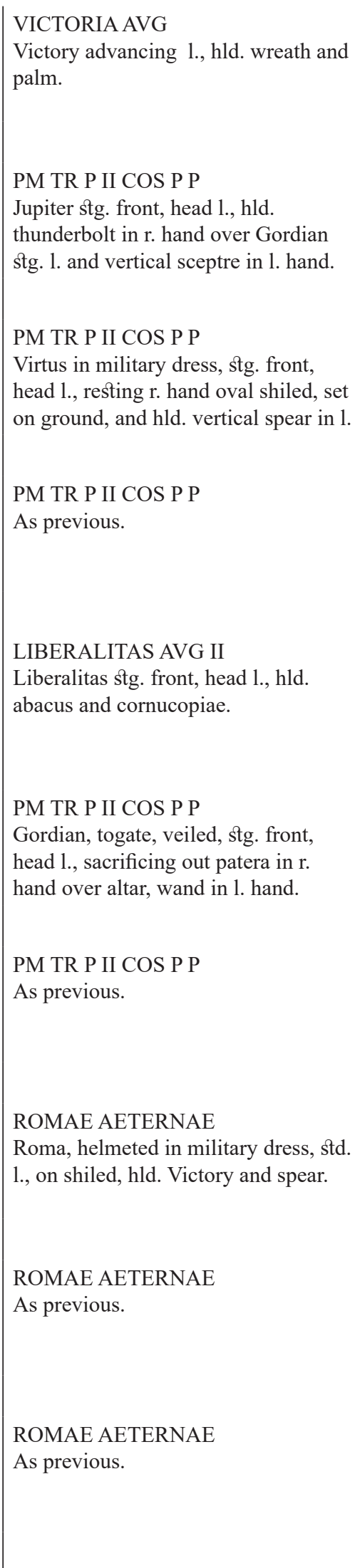

\begin{tabular}{|c|c|c|}
\hline $\begin{array}{l}\text { RIC IV/ } \\
\text { III, } 5 .\end{array}$ & $238-239$ & 6012 \\
\hline $\begin{array}{l}\text { RIC IV/ } \\
\text { III, } 16\end{array}$ & 239 & 5990 \\
\hline $\begin{array}{l}\text { RIC IV/ } \\
\text { III, } 20 .\end{array}$ & 239 & 6005 \\
\hline $\begin{array}{l}\text { RIC IV/ } \\
\text { III, } 20 .\end{array}$ & 239 & 5978 \\
\hline $\begin{array}{l}\text { RIC IV/ } \\
\text { III, } 36 .\end{array}$ & 240 & 6009 \\
\hline $\begin{array}{l}\text { RIC IV/ } \\
\text { III, } 37 .\end{array}$ & 240 & 5974 \\
\hline $\begin{array}{l}\text { RIC IV/ } \\
\text { III, } 37 .\end{array}$ & 240 & 5969 \\
\hline $\begin{array}{l}\text { RIC IV/ } \\
\text { III, } 38\end{array}$ & 240 & 6206 \\
\hline $\begin{array}{l}\text { RIC IV/ } \\
\text { III, } 38\end{array}$ & 240 & 5966 \\
\hline $\begin{array}{l}\text { RIC IV/ } \\
\text { III, } 38\end{array}$ & 240 & 5972 \\
\hline
\end{tabular}




\begin{tabular}{|c|c|c|c|c|c|c|}
\hline 59 & \begin{tabular}{|l|} 
ANT. \\
3.26 \\
21.5 \\
1
\end{tabular} & $\begin{array}{l}\text { IMP CAES M ANT } \\
\text { GORDIANVS AVG } \\
\text { As previous }\end{array}$ & $\begin{array}{l}\text { ROMAE AETERNAE } \\
\text { As previous. }\end{array}$ & $\begin{array}{l}\text { RIC IV/ } \\
\text { III, } 38\end{array}$ & 240 & 6020 \\
\hline 60 & $\begin{array}{l}\text { AnT. } \\
4.44 \\
22.9 \\
1\end{array}$ & $\begin{array}{l}\text { IMP CAES } \\
\text { GORDIANVS PIVS } \\
\text { AVG } \\
\text { As previous. }\end{array}$ & $\begin{array}{l}\text { VIRTVS AVG } \\
\text { Virtus helmeted, in military dress, stg. } \\
\text { front, head 1., hld. branch and spear; } \\
\text { shiled rests against r. foot. }\end{array}$ & $\begin{array}{l}\text { RIC IV/ } \\
\text { III, } 56 .\end{array}$ & 240 & 5982 \\
\hline 61 & $\begin{array}{l}\text { ANT. } \\
4.12 \\
22.2 \\
1\end{array}$ & $\begin{array}{l}\text { IMP GORDIANVS } \\
\text { PIVS FEL AVG } \\
\text { As previous. }\end{array}$ & $\begin{array}{l}\text { AEQVITAS AVG } \\
\text { Aequitas stg. front, head 1., hld. scales } \\
\text { and cornucopiae. }\end{array}$ & $\begin{array}{l}\text { RIC IV/ } \\
\text { III, } 63\end{array}$ & 240 & 5986 \\
\hline 62 & $\begin{array}{l}\text { ANT. } \\
5.12 \\
22.8 \\
7\end{array}$ & $\begin{array}{l}\text { IMP GORDIANVS } \\
\text { PIVS FEL AVG } \\
\text { As previous. }\end{array}$ & $\begin{array}{l}\text { CONCORDIA MILIT } \\
\text { Concordia std. } 1 . \text { on throne, hld. } \\
\text { patera and double cornucopiae. }\end{array}$ & $\begin{array}{l}\text { RIC IV/ } \\
\text { III, } 65 .\end{array}$ & 240 & 5962 \\
\hline 63 & $\begin{array}{l}\text { ANT. } \\
3.52 \\
21.9 \\
1\end{array}$ & $\begin{array}{l}\text { IMP GORDIANVS PIVS } \\
\text { FEL AVG } \\
\text { As previous. }\end{array}$ & $\begin{array}{l}\text { CONCORDIA MILIT } \\
\text { As previous. }\end{array}$ & $\begin{array}{l}\text { RIC IV/ } \\
\text { III, } 65 .\end{array}$ & 240 & 5950 \\
\hline 64 & $\begin{array}{l}\text { ANT. } \\
4.38 \\
23.8 \\
5\end{array}$ & $\begin{array}{l}\text { IMP GORDIANVS PIVS } \\
\text { FEL AVG } \\
\text { As previous. }\end{array}$ & $\begin{array}{l}\text { ROMAE AE-TERNAE } \\
\text { Roma, helmeted in military dress, std. } \\
\text { 1., on shiled, hld. Victory and spear. }\end{array}$ & $\begin{array}{l}\text { RIC IV/ } \\
\text { III, } 70\end{array}$ & 240 & 6019 \\
\hline 65 & $\begin{array}{l}\text { ANT. } \\
4.50 \\
24.5 \\
7\end{array}$ & $\begin{array}{l}\text { IMP GORDIANVS PIVS } \\
\text { FEL AVG } \\
\text { As previous. }\end{array}$ & $\begin{array}{l}\text { ROMAE AE-TERNAE } \\
\text { As previous. }\end{array}$ & $\begin{array}{l}\text { RIC IV/ } \\
\text { III, } 70\end{array}$ & 240 & 5970 \\
\hline 66 & $\begin{array}{l}\text { ANT. } \\
3.46 \\
24.2 \\
12\end{array}$ & $\begin{array}{l}\text { IMP GORDIANVS PIVS } \\
\text { FEL AVG } \\
\text { As previous. }\end{array}$ & $\begin{array}{l}\text { VIRTVS AVG } \\
\text { Virtus helmeted, in military dress, stg. } \\
\text { front, head 1., hld. branch and spear; } \\
\text { shiled rests against r. foot. }\end{array}$ & $\begin{array}{l}\text { RIC IV/ } \\
\text { III, } 71 .\end{array}$ & 240 & 5965 \\
\hline 67 & $\begin{array}{l}\text { AnT. } \\
4.48 \\
23.2 \\
1\end{array}$ & $\begin{array}{l}\text { IMP GORDIANVS PIVS } \\
\text { FEL AVG } \\
\text { As previous. }\end{array}$ & $\begin{array}{l}\text { VIR-TV-S AVG } \\
\text { As previous. }\end{array}$ & $\begin{array}{l}\text { RIC IV/ } \\
\text { III, } 71 .\end{array}$ & 240 & 6008 \\
\hline 68 & $\begin{array}{l}\text { ANT. } \\
3.57 \\
22.2 \\
7\end{array}$ & $\begin{array}{l}\text { IMP GORDIANVS PIVS } \\
\text { FEL AVG } \\
\text { Bust rad., dr., cuir., r. }\end{array}$ & $\begin{array}{l}\text { AETER-N-ITATI AVG } \\
\text { Sol stg. front, head 1., raising r. hand } \\
\text { and hld. globe. }\end{array}$ & $\begin{array}{l}\text { RIC IV/ } \\
\text { III, } 83\end{array}$ & $241-243$ & 5981 \\
\hline 69 & $\begin{array}{l}\text { AnT. } \\
4.06 \\
22.8 \\
7\end{array}$ & $\begin{array}{l}\text { IMP GORDIANVS PIVS } \\
\text { FEL AVG } \\
\text { As previous. }\end{array}$ & $\begin{array}{l}\text { AETER-NITATI AVG } \\
\text { As previous. }\end{array}$ & $\begin{array}{l}\text { RIC IV/ } \\
\text { III, } 83\end{array}$ & $241-243$ & 6026 \\
\hline
\end{tabular}




\begin{tabular}{|c|c|c|c|c|c|c|}
\hline 70 & \begin{tabular}{|l} 
ANT. \\
3.83 \\
25.6 \\
1
\end{tabular} & $\begin{array}{l}\text { IMP GORDIANVS PIVS } \\
\text { FEL AVG } \\
\text { As previous. }\end{array}$ & $\begin{array}{l}\text { AETER-NITATI AVG } \\
\text { As previous. }\end{array}$ & $\begin{array}{l}\text { RIC IV/ } \\
\text { III, } 83\end{array}$ & $241-243$ & 5959 \\
\hline 71 & \begin{tabular}{|l} 
ANT. \\
3.07 \\
23.9 \\
1
\end{tabular} & $\begin{array}{l}\text { IMP GORDIANVS PIVS } \\
\text { FEL AVG } \\
\text { As previous. }\end{array}$ & $\begin{array}{l}\text { AETER-NITATI AVG } \\
\text { As previous. }\end{array}$ & $\begin{array}{l}\text { RIC IV/ } \\
\text { III, } 83\end{array}$ & $241-243$ & 5954 \\
\hline 72 & \begin{tabular}{|l} 
ANT. \\
4.24 \\
23.0 \\
6
\end{tabular} & $\begin{array}{l}\text { IMP GORDIANVS PIVS } \\
\text { FEL AVG } \\
\text { As previous. }\end{array}$ & $\begin{array}{l}\text { AETER-NITATI AVG } \\
\text { As previous. }\end{array}$ & $\begin{array}{l}\text { RIC IV/ } \\
\text { III, } 83\end{array}$ & $241-243$ & 6013 \\
\hline 73 & \begin{tabular}{|l} 
ANT. \\
4.17 \\
23.2 \\
7
\end{tabular} & $\begin{array}{l}\text { IMP GORDIANVS PIVS } \\
\text { FEL AVG } \\
\text { As previous. }\end{array}$ & $\begin{array}{l}\text { IOVI STATORI } \\
\text { Jupiter stg. front, head r., hld. long } \\
\text { sceptre and thunderbolt. }\end{array}$ & $\begin{array}{l}\text { RIC IV/ } \\
\text { III, } 84 .\end{array}$ & $241-243$ & 6017 \\
\hline 74 & \begin{tabular}{|l} 
Ant. \\
4.04 \\
23.3 \\
7
\end{tabular} & $\begin{array}{l}\text { IMP GORDIANVS PIVS } \\
\text { FEL AVG } \\
\text { As previous. }\end{array}$ & $\begin{array}{l}\text { IOVI STATORI } \\
\text { As previous. }\end{array}$ & $\begin{array}{l}\text { RIC IV/ } \\
\text { III, } 84 .\end{array}$ & $241-243$ & 5958 \\
\hline 75 & \begin{tabular}{|l} 
ANT. \\
3.71 \\
22.9 \\
7
\end{tabular} & $\begin{array}{l}\text { IMP GORDIANVS PIVS } \\
\text { FEL AVG } \\
\text { As previous. }\end{array}$ & $\begin{array}{l}\text { IOVI STATORI } \\
\text { As previous. }\end{array}$ & $\begin{array}{l}\text { RIC IV/ } \\
\text { III, } 84 .\end{array}$ & $241-243$ & 6006 \\
\hline 76 & \begin{tabular}{|l} 
ANT. \\
4.26 \\
23.1 \\
12
\end{tabular} & $\begin{array}{l}\text { IMP GORDIANVS PIVS } \\
\text { FEL AVG } \\
\text { As previous. }\end{array}$ & $\begin{array}{l}\text { IOVI S-TATORI } \\
\text { As previous. }\end{array}$ & $\begin{array}{l}\text { RIC IV/ } \\
\text { III, } 84 .\end{array}$ & $241-243$ & 5975 \\
\hline 77 & \begin{tabular}{|l} 
Ant. \\
4.21 \\
23.2 \\
6
\end{tabular} & $\begin{array}{l}\text { IMP GORDIANVS PIVS } \\
\text { FEL AVG } \\
\text { As previous. }\end{array}$ & $\begin{array}{l}\text { IOVI S-TATORI } \\
\text { As previous. }\end{array}$ & $\begin{array}{l}\text { RIC IV/ } \\
\text { III, } 84 .\end{array}$ & $241-243$ & 6028 \\
\hline 78 & \begin{tabular}{|l} 
ANT. \\
3.44 \\
23.6 \\
1
\end{tabular} & $\begin{array}{l}\text { IMP GORDIANVS PIVS } \\
\text { FEL AVG } \\
\text { As previous. }\end{array}$ & $\begin{array}{l}\text { LAETITIA AVG [N] } \\
\text { Laetitia stg. 1., hld. wreath and } \\
\text { anchor. }\end{array}$ & $\begin{array}{l}\text { RIC IV/ } \\
\text { III, } 86 .\end{array}$ & $241-243$ & 5977 \\
\hline 79 & \begin{tabular}{|l} 
ANT. \\
3.36 \\
23.0 \\
5
\end{tabular} & $\begin{array}{l}\text { IMP GORDIANVS PIVS } \\
\text { FEL AVG } \\
\text { As previous. }\end{array}$ & $\begin{array}{l}\text { LAETITIA AVG N } \\
\text { As previous. }\end{array}$ & $\begin{array}{l}\text { RIC IV/ } \\
\text { III, } 86 .\end{array}$ & $241-243$ & 5988 \\
\hline 80 & \begin{tabular}{|l} 
ANT. \\
4.57 \\
23.4 \\
6
\end{tabular} & $\begin{array}{l}\text { IMP GORDIANVS PIVS } \\
\text { FEL AVG } \\
\text { As previous. }\end{array}$ & $\begin{array}{l}\text { LAETITIA AVG N } \\
\text { As previous. }\end{array}$ & $\begin{array}{l}\text { RIC IV/ } \\
\text { III, } 86 .\end{array}$ & $241-243$ & 6023 \\
\hline
\end{tabular}




\begin{tabular}{|c|c|c|c|c|c|c|}
\hline 81 & \begin{tabular}{|l|} 
ANT. \\
4.19 \\
23.2 \\
12
\end{tabular} & $\begin{array}{l}\text { IMP GORDIANVS PIVS } \\
\text { FEL AVG } \\
\text { As previous. }\end{array}$ & $\begin{array}{l}\text { P M TR P III COS II } \\
\text { Apollo, bare to waist, std. 1., hld. } \\
\text { branch and resting elbow on lyre. }\end{array}$ & \begin{tabular}{|l|} 
RIC IV/ \\
III, 87.
\end{tabular} & |241-243 & $\mid 5953$ \\
\hline 82 & \begin{tabular}{|l} 
ANT. \\
4.36 \\
22.3 \\
1
\end{tabular} & $\begin{array}{l}\text { IMP GORDIANVS PIVS } \\
\text { FEL AVG } \\
\text { As previous. }\end{array}$ & $\begin{array}{l}\text { P M TR P IIII COS II P P } \\
\text { As previous. }\end{array}$ & $\begin{array}{l}\text { RIC IV/ } \\
\text { III, } 88\end{array}$ & 241-243 & 5979 \\
\hline 83 & \begin{tabular}{|l} 
Ant. \\
3.66 \\
25.1 \\
7
\end{tabular} & $\begin{array}{l}\text { IMP GORDIANVS PIVS } \\
\text { FEL AVG } \\
\text { As previous. }\end{array}$ & $\begin{array}{l}\text { Rev. P M TR P V COS II P P } \\
\text { As previous. }\end{array}$ & \begin{tabular}{|l} 
RIC IV/ \\
III, 88
\end{tabular} & $241-243$ & 5952 \\
\hline 84 & \begin{tabular}{|l} 
ANT. \\
4.24 \\
22.0 \\
7
\end{tabular} & $\begin{array}{l}\text { IMP GORDIANVS PIVS } \\
\text { FEL AVG } \\
\text { As previous. }\end{array}$ & $\begin{array}{l}\text { P M TR P V COS II P P } \\
\text { As previous. }\end{array}$ & $\begin{array}{l}\text { RIC IV/ } \\
\text { III, } 89 .\end{array}$ & $241-243$ & 5984 \\
\hline 85 & \begin{tabular}{|l} 
Ant. \\
3.46 \\
23.8 \\
5
\end{tabular} & $\begin{array}{l}\text { IMP GORDIANVS PIVS } \\
\text { FEL AVG } \\
\text { As previous. }\end{array}$ & $\begin{array}{l}\text { PM TR P III COS II P P } \\
\text { Gordian in military dress, stg. r., hld. } \\
\text { transverse spear and globe. }\end{array}$ & $\begin{array}{l}\text { RIC IV/ } \\
\text { III, } 91 .\end{array}$ & 241-243 & 5949 \\
\hline 86 & \begin{tabular}{|l} 
ANT. \\
4.30 \\
23.2 \\
1
\end{tabular} & $\begin{array}{l}\text { IMP GORDIANVS PIVS } \\
\text { FEL AVG } \\
\text { As previous. }\end{array}$ & $\begin{array}{l}\text { PM TR P III COS II P P } \\
\text { As previous. }\end{array}$ & $\begin{array}{l}\text { RIC IV/ } \\
\text { III, } 91 .\end{array}$ & $241-243$ & 5956 \\
\hline 87 & \begin{tabular}{|l} 
ANT. \\
4.32 \\
21.6 \\
1
\end{tabular} & $\begin{array}{l}\text { IMP GORDIANVS PIVS } \\
\text { FEL AVG } \\
\text { As previous. }\end{array}$ & $\begin{array}{l}\text { PM TR P IIII COS II P P } \\
\text { As previous. }\end{array}$ & $\begin{array}{l}\text { RIC IV/ } \\
\text { III, } 92 .\end{array}$ & $241-243$ & 6025 \\
\hline 88 & \begin{tabular}{|l} 
ANT. \\
4.13 \\
21.4 \\
1
\end{tabular} & $\begin{array}{l}\text { IMP GORDIANVS PIVS } \\
\text { FEL AVG } \\
\text { As previous. }\end{array}$ & $\begin{array}{l}\text { PM TR P IIII COS II P P } \\
\text { As previous. }\end{array}$ & $\begin{array}{l}\text { RIC IV/ } \\
\text { III, } 92 .\end{array}$ & $241-243$ & 6002 \\
\hline 89 & \begin{tabular}{|l} 
ANT. \\
4.19 \\
22.5 \\
1
\end{tabular} & $\begin{array}{l}\text { IMP GORDIANVS PIVS } \\
\text { FEL AVG } \\
\text { As previous. }\end{array}$ & $\begin{array}{l}\text { PM TR P IIII COS II P P } \\
\text { As previous. }\end{array}$ & $\begin{array}{l}\text { RIC IV/ } \\
\text { III, } 92 .\end{array}$ & $241-243$ & 6010 \\
\hline 90 & \begin{tabular}{|l} 
ANT. \\
3.26 \\
20.8 \\
12
\end{tabular} & $\begin{array}{l}\text { IMP GORDIANVS PIVS } \\
\text { FEL AVG } \\
\text { As previous. }\end{array}$ & $\begin{array}{l}\text { PM TR P IIII COS II P P } \\
\text { As previous. }\end{array}$ & $\begin{array}{l}\text { RIC IV/ } \\
\text { III, } 92 .\end{array}$ & 241-243 & 6011 \\
\hline 91 & \begin{tabular}{|l} 
ANT. \\
3.82 \\
23.6 \\
1
\end{tabular} & $\begin{array}{l}\text { IMP GORDIANVS PIVS } \\
\text { FEL AVG } \\
\text { As previous. }\end{array}$ & $\begin{array}{l}\text { PM TR P V COS II P P } \\
\text { As previous. }\end{array}$ & $\begin{array}{l}\text { RIC IV/ } \\
\text { III, } 93 .\end{array}$ & 241-243 & 6015 \\
\hline
\end{tabular}



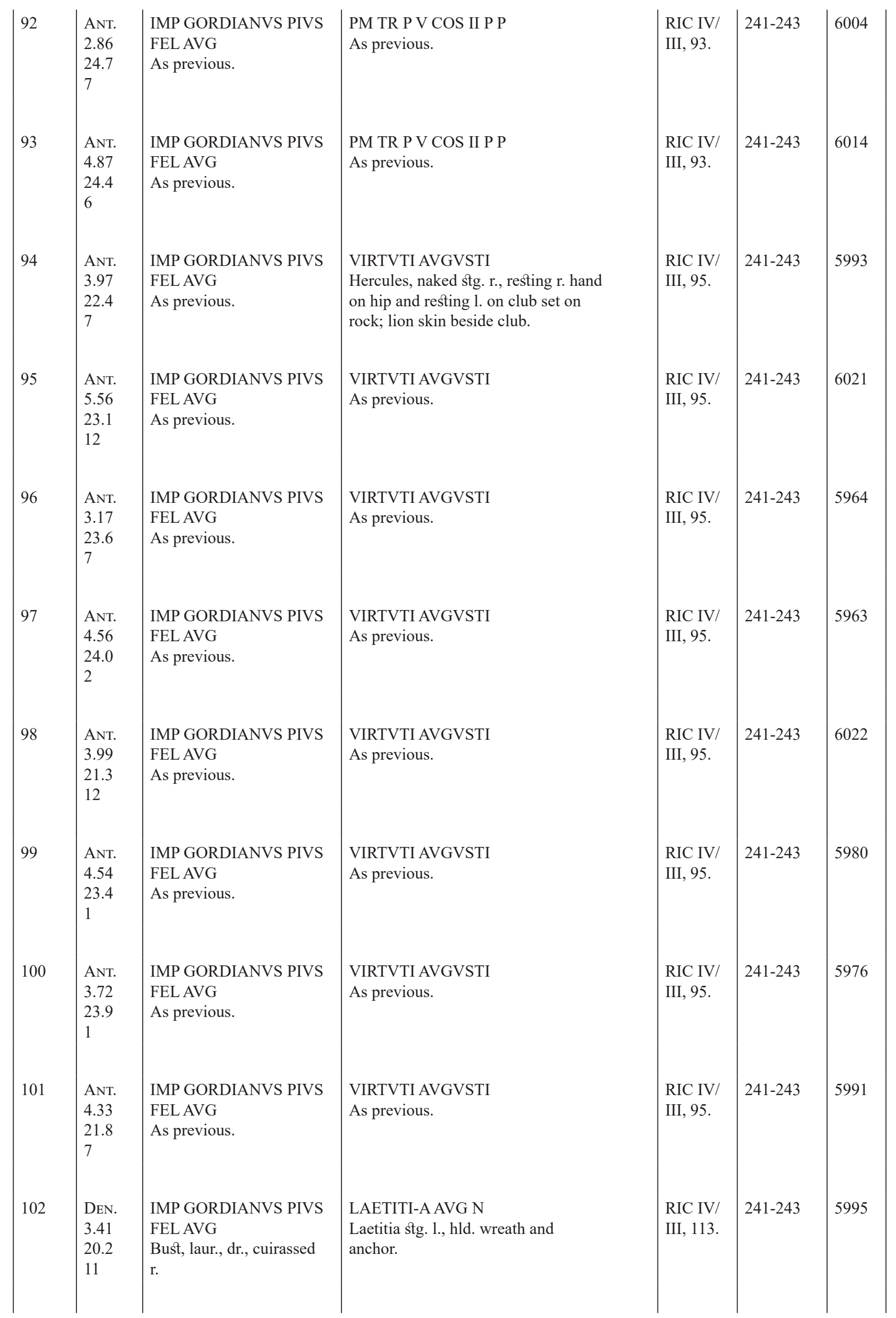


\begin{tabular}{|c|c|c|c|c|c|c|}
\hline 103 & \begin{tabular}{|l} 
DEN. \\
2.27 \\
20.8 \\
1
\end{tabular} & $\begin{array}{l}\text { IMP GORDIANVS PIVS } \\
\text { FEL AVG } \\
\text { As previous. }\end{array}$ & $\begin{array}{l}\text { P M TR P III COS II P P } \\
\text { Apollo std. l., hld. branch and } \\
\text { resting 1. elbow on lyre. }\end{array}$ & $\begin{array}{l}\text { RIC IV/ } \\
\text { III, } 113 .\end{array}$ & $241-243$ & 5998 \\
\hline 104 & \begin{tabular}{|l} 
DEN. \\
3.03 \\
20.1 \\
6
\end{tabular} & $\begin{array}{l}\text { IMP GORDIANVS PIVS } \\
\text { FEL AVG } \\
\text { As previous. }\end{array}$ & $\begin{array}{l}\text { SALVS AVGVSTI } \\
\text { Salus stg. r., feeding snake held in } \\
\text { arms. }\end{array}$ & $\begin{array}{l}\text { RIC } \\
\text { IV/III, } \\
129 \mathrm{~A} .\end{array}$ & 241 & 6007 \\
\hline 105 & \begin{tabular}{|l} 
DEN. \\
4.10 \\
23.5 \\
1
\end{tabular} & $\begin{array}{l}\text { IMP GORDIANVS PIVS } \\
\text { FEL AVG } \\
\text { As previous. }\end{array}$ & $\begin{array}{l}\text { DIANA LVCI-FERA } \\
\text { Diana stg. r., hld. long torch. }\end{array}$ & $\begin{array}{l}\text { RIC IV/ } \\
\text { III, } 127 .\end{array}$ & 241 & 5996 \\
\hline 106 & \begin{tabular}{|l} 
DEN. \\
3.00 \\
20.3 \\
1
\end{tabular} & $\begin{array}{l}\text { IMP GORDIANVS PIVS } \\
\text { FEL AVG } \\
\text { As previous. }\end{array}$ & $\begin{array}{l}\text { VENVS VICTRIX } \\
\text { Venus stg. l., hld. helmet and sceptre } \\
\text { and leaning on shiled. }\end{array}$ & $\begin{array}{l}\text { RIC IV/ } \\
\text { III, } 131 .\end{array}$ & 241 & 5997 \\
\hline 107 & \begin{tabular}{|l} 
AnT. \\
4.28 \\
23.7 \\
1
\end{tabular} & $\begin{array}{l}\text { IMP GORDIANVS PIVS } \\
\text { FEL AVG } \\
\text { As previous. }\end{array}$ & $\begin{array}{l}\text { FELICITA TEMPORVM } \\
\text { Felicitas stg. 1., hld. caduceus } \\
\text { and cornucopiae. }\end{array}$ & $\begin{array}{l}\text { RIC IV/ } \\
\text { III, } 142 .\end{array}$ & $243-244$ & 5955 \\
\hline 108 & \begin{tabular}{|l} 
ANT. \\
5.27 \\
24.2 \\
1
\end{tabular} & $\begin{array}{l}\text { IMP GORDIANVS PIVS } \\
\text { FEL AVG } \\
\text { As previous. }\end{array}$ & $\begin{array}{l}\text { FELICIT TEMP } \\
\text { Felicitas stg. 1., hld. long caduceus } \\
\text { and cornucopiae. }\end{array}$ & $\begin{array}{l}\text { RIC IV/ } \\
\text { III, } 140 .\end{array}$ & $243-244$ & 6016 \\
\hline 109 & \begin{tabular}{|l} 
ANT. \\
3.73 \\
21.6 \\
7
\end{tabular} & $\begin{array}{l}\text { IMP GORDIANVS PIVS } \\
\text { FEL AVG } \\
\text { As previous. }\end{array}$ & $\begin{array}{l}\text { FELICIT TEMP } \\
\text { As previous. }\end{array}$ & $\begin{array}{l}\text { RIC IV/ } \\
\text { III, } 140 .\end{array}$ & $243-244$ & 5983 \\
\hline 110 & \begin{tabular}{|l} 
AnT. \\
4.23 \\
26.4 \\
1
\end{tabular} & $\begin{array}{l}\text { IMP GORDIANVS PIVS } \\
\text { FEL AVG } \\
\text { As previous. }\end{array}$ & $\begin{array}{l}\text { FELICIT TEMPOR } \\
\text { As previous. }\end{array}$ & $\begin{array}{l}\text { RIC IV/ } \\
\text { III, } 141 .\end{array}$ & $243-244$ & 5973 \\
\hline 111 & \begin{tabular}{|l|} 
ANT. \\
3.91 \\
23.1 \\
1
\end{tabular} & $\begin{array}{l}\text { IMP GORDIANVS PIVS } \\
\text { FEL AVG } \\
\text { As previous. }\end{array}$ & $\begin{array}{l}\text { FORTVNA REDVX } \\
\text { Fortuna std. 1., hld. rudder and } \\
\text { cornucopiae; under seat, wheel. }\end{array}$ & $\begin{array}{l}\text { RIC IV/ } \\
\text { III, } 144 .\end{array}$ & $243-244$ & 6000 \\
\hline 112 & \begin{tabular}{|l} 
ANT. \\
4.75 \\
23.2 \\
1
\end{tabular} & $\begin{array}{l}\text { IMP GORDIANVS PIVS } \\
\text { FEL AVG } \\
\text { As previous. }\end{array}$ & $\begin{array}{l}\text { FORTVNA REDVX } \\
\text { As previous (without a wheel). }\end{array}$ & $\begin{array}{l}\text { RIC IV/ } \\
\text { III, } 144 .\end{array}$ & $243-244$ & 5968 \\
\hline 113 & \begin{tabular}{|l} 
ANT. \\
4.36 \\
23.1 \\
7
\end{tabular} & $\begin{array}{l}\text { IMP GORDIANVS PIVS } \\
\text { FEL AVG } \\
\text { As previous. }\end{array}$ & $\begin{array}{l}\text { MARS PROPVG } \\
\text { Mars hastening r., hld. trasverse } \\
\text { spear and shield. }\end{array}$ & $\begin{array}{l}\text { RIC IV/ } \\
\text { III, } 145 .\end{array}$ & $243-244$ & 5992 \\
\hline
\end{tabular}




\begin{tabular}{|c|c|c|c|c|c|c|}
\hline 114 & \begin{tabular}{|l|} 
AnT. \\
4.39 \\
22.4 \\
6
\end{tabular} & $\begin{array}{l}\text { IMP GORDIANVS PIVS } \\
\text { FEL AVG } \\
\text { As previous. }\end{array}$ & $\begin{array}{l}\text { MARS PROPVG } \\
\text { As previous. }\end{array}$ & \begin{tabular}{|l} 
RIC IV/ \\
III, 145.
\end{tabular} & 243-244 & 6024 \\
\hline 115 & $\begin{array}{l}\text { AnT. } \\
3.65 \\
23.0 \\
7\end{array}$ & $\begin{array}{l}\text { IMP GORDIANVS PIVS } \\
\text { FEL AVG } \\
\text { As previous. }\end{array}$ & $\begin{array}{l}\text { PROVID AVG } \\
\text { Providentia stg. 1, hld. wand over } \\
\text { globe and sceptre. }\end{array}$ & $\begin{array}{l}\text { RIC IV/ } \\
\text { III, 148 }\end{array}$ & $243-244$ & 5967 \\
\hline 116 & $\begin{array}{l}\text { ANT. } \\
4.82 \\
23.7 \\
7\end{array}$ & $\begin{array}{l}\text { IMP GORDIANVS PIVS } \\
\text { FEL AVG } \\
\text { As previous. }\end{array}$ & $\begin{array}{l}\text { PROVID AVG } \\
\text { As previous. }\end{array}$ & $\begin{array}{l}\text { RIC IV/ } \\
\text { III, } 148\end{array}$ & $243-244$ & 6001 \\
\hline 117 & $\begin{array}{l}\text { AnT. } \\
3.87 \\
22.1 \\
11\end{array}$ & $\begin{array}{l}\text { IMP GORDIANVS PIVS } \\
\text { FEL AVG } \\
\text { As previous. }\end{array}$ & $\begin{array}{l}\text { PROVID AVG } \\
\text { As previous. }\end{array}$ & $\begin{array}{l}\text { RIC IV/ } \\
\text { III, } 148\end{array}$ & $243-244$ & 5989 \\
\hline 118 & $\begin{array}{l}\text { AnT. } \\
3.66 \\
24.2 \\
11\end{array}$ & $\begin{array}{l}\text { IMP GORDIANVS PIVS } \\
\text { FEL AVG } \\
\text { As previous. }\end{array}$ & $\begin{array}{l}\text { SECV-RIT PERP } \\
\text { Securitas stg. l., legs crossed, hld. } \\
\text { sceptre and resting } 1 \text {. arm on column. }\end{array}$ & $\begin{array}{l}\text { RIC IV/ } \\
\text { III, } 151 .\end{array}$ & $243-244$ & 5961 \\
\hline 119 & $\begin{array}{l}\text { AnT. } \\
2.69 \\
21.5 \\
11\end{array}$ & $\begin{array}{l}\text { IMP GORDIANVS PIVS } \\
\text { FEL AVG } \\
\text { As previous. }\end{array}$ & $\begin{array}{l}\text { SECVRIT PERP } \\
\text { As previous. }\end{array}$ & $\begin{array}{l}\text { RIC IV/ } \\
\text { III, } 151 .\end{array}$ & $243-244$ & 5960 \\
\hline 120 & $\begin{array}{l}\text { ANT. } \\
3.07 \\
24.1 \\
1\end{array}$ & $\begin{array}{l}\text { IMP GORDIANVS PIVS } \\
\text { FEL AVG } \\
\text { As previous. }\end{array}$ & $\begin{array}{l}\text { SECVRITAS PERPETVA } \\
\text { Securitas stg. 1., legs crossed, hld. } \\
\text { sceptre and resting } 1 . \text { arm on } \\
\text { column. }\end{array}$ & $\begin{array}{l}\text { RIC IV/ } \\
\text { III, } \\
153 .\end{array}$ & $243-244$ & 5994 \\
\hline 121 & \begin{tabular}{|l} 
AnT. \\
4.23 \\
21.4 \\
1
\end{tabular} & $\begin{array}{l}\text { IMP GORDIANVS PIVS } \\
\text { FEL AVG } \\
\text { As previous. }\end{array}$ & $\begin{array}{l}\text { VICTORIA AETERNA } \\
\text { Victory stg. 1. leaning on shield } \\
\text { and hld. palm; under shield, } \\
\text { captive. }\end{array}$ & $\begin{array}{l}\text { RIC IV/ } \\
\text { III, } 156 .\end{array}$ & $243-244$ & 5971 \\
\hline 122 & $\begin{array}{l}\text { AnT. } \\
4.39 \\
22.4 \\
1\end{array}$ & $\begin{array}{l}\text { IMP GORDIANVS PIVS } \\
\text { FEL AVG } \\
\text { As previous. }\end{array}$ & $\begin{array}{l}\text { VICTORIA AETERNA } \\
\text { As previous. }\end{array}$ & $\begin{array}{l}\text { RIC IV/ } \\
\text { III, } 156 .\end{array}$ & $243-244$ & 6003 \\
\hline
\end{tabular}

(cat. 122-130)

Mint of Antioch

\begin{tabular}{|l|l|l}
123 & ANT. & IMP CAES M ANT \\
& 3.96 & GORDIANVS AVG \\
& 20.9 & As previous. \\
& &
\end{tabular}

FIDE-S MILITVM
Fides std. 1., hld. standard and
cornucopiae. 


\begin{tabular}{|c|c|c|}
\hline 124 & $\begin{array}{l}\text { ANT. } \\
4.68 \\
23.0 \\
12\end{array}$ & $\begin{array}{l}\text { IMP CAES M ANT } \\
\text { GORDIANVS AVG } \\
\text { As previous. }\end{array}$ \\
\hline 125 & $\begin{array}{l}\text { ANT. } \\
5.49 \\
22.8 \\
1\end{array}$ & $\begin{array}{l}\text { IMP CAES M ANT } \\
\text { GORDIANVS AVG } \\
\text { As previous. }\end{array}$ \\
\hline 126 & $\begin{array}{l}\text { ANT. } \\
4.51 \\
22.0 \\
6\end{array}$ & $\begin{array}{l}\text { IMP GORDIANVS PIVS } \\
\text { FEL AVG } \\
\text { As previous. }\end{array}$ \\
\hline 127 & $\begin{array}{l}\text { ANT. } \\
4.16 \\
20.9 \\
6\end{array}$ & $\begin{array}{l}\text { IMP GORDIANVS PIVS } \\
\text { FEL AVG } \\
\text { As previous. }\end{array}$ \\
\hline 128 & $\begin{array}{l}\text { ANT. } \\
4.07 \\
22.8 \\
1\end{array}$ & $\begin{array}{l}\text { IMP GORDIANVS PIVS } \\
\text { FEL AVG } \\
\text { As previous. }\end{array}$ \\
\hline 129 & $\begin{array}{l}\text { ANT. } \\
3.38 \\
23.2 \\
7\end{array}$ & $\begin{array}{l}\text { IMP GORDIANVS PIVS } \\
\text { FEL AVG } \\
\text { As previous. }\end{array}$ \\
\hline 130 & $\begin{array}{l}\text { ANT. } \\
4.12 \\
25.0 \\
6\end{array}$ & $\begin{array}{l}\text { IMP GORDIANVS PIVS } \\
\text { FEL AVG } \\
\text { As previous. }\end{array}$ \\
\hline
\end{tabular}

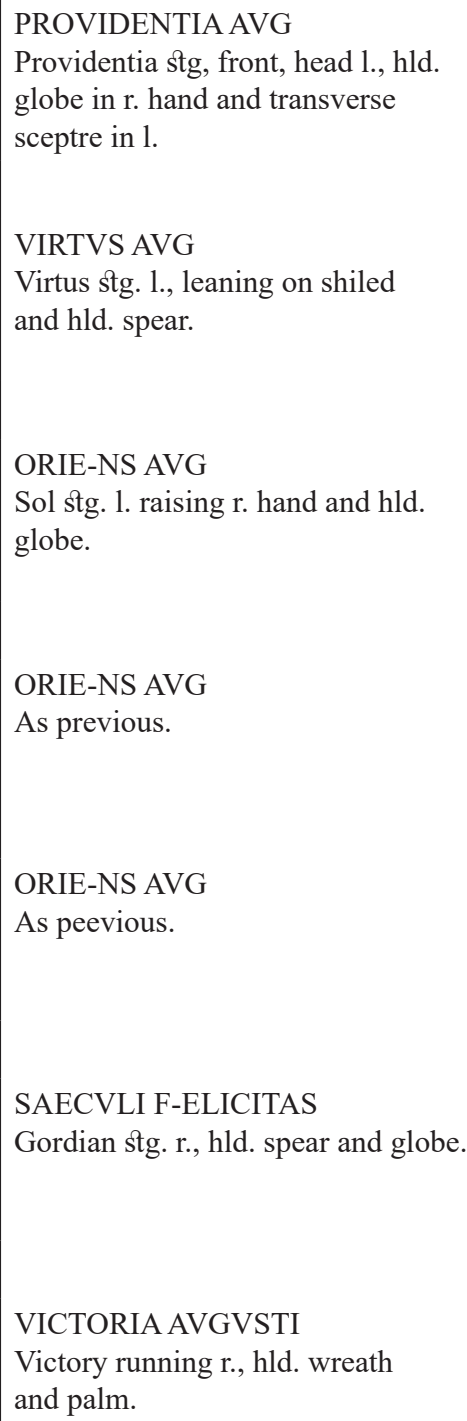

RIC IV/

\section{PHILIP I}

(cat. 130-169)

\section{Mint of Rome}

\begin{tabular}{|l|l|l|}
131 & ANT. & IMP M IVL PHILIPPVS \\
& 4.52 & AVG \\
& 23.7 & As previous. \\
& 7 & \\
& & \\
& ANT. & IMP M IVL PHILIPPVS \\
& 3.55 & AVG \\
& 23.4 & As previous. \\
& 7 & \\
& & \\
& ANT. & IMP M IVL PHILIPPVS \\
& 4.44 & AVG \\
& 23.2 & As previous. \\
& 7 &
\end{tabular}

PM TR P II COS P P

Philip I std. 1., on curule chair, hld. globe and short scepter.

\section{PM TR P II COS P P}

As previous.

P M TR P III COS P P

Felicitas stg. 1., hld. caduceus and cornucopia.

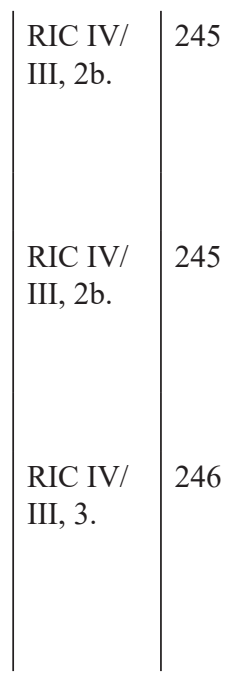

6071

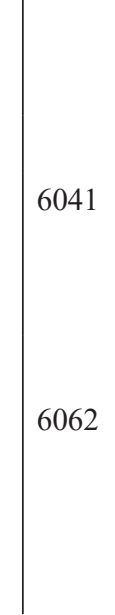




\begin{tabular}{|c|c|c|c|c|c|c|}
\hline 134 & $\begin{array}{l}\text { ANT. } \\
5.30 \\
21.7 \\
7\end{array}$ & $\begin{array}{l}\text { IMP M IVL PHILIPPVS } \\
\text { AVG } \\
\text { As previous. }\end{array}$ & $\begin{array}{l}\text { P M TR P IIII COS II P P } \\
\text { As previous. }\end{array}$ & $\begin{array}{l}\text { RIC IV/ } \\
\text { III, } 4 .\end{array}$ & 247 & 6051 \\
\hline 135 & $\begin{array}{l}\text { ANT. } \\
3.65 \\
23.7 \\
7\end{array}$ & $\begin{array}{l}\text { IMP PHILIPPVS AVG } \\
\text { As previous. }\end{array}$ & $\begin{array}{l}\text { PM TR P V COS III P P } \\
\text { Mars stg. 1., hld. branch and resting } 1 \text {. } \\
\text { hand on shield; spear against } 1 \text {. arm: } \\
\text { A, 1. in filed. }\end{array}$ & $\begin{array}{l}\text { RIC IV/ } \\
\text { III, } 7 .\end{array}$ & 248 & 6064 \\
\hline 136 & $\begin{array}{l}\text { ANT. } \\
4.22 \\
21.5 \\
7\end{array}$ & $\begin{array}{l}\text { IMP PHILIPPVS AVG } \\
\text { As previous. }\end{array}$ & $\begin{array}{l}\text { SAECVLARES AVGG } \\
\text { Lion walking r.; in ex. I. }\end{array}$ & $\begin{array}{l}\text { RIC IV/ } \\
\text { III, } 12 .\end{array}$ & 248 & 6070 \\
\hline 137 & $\begin{array}{l}\text { ANT. } \\
3.23 \\
23.0 \\
7\end{array}$ & $\begin{array}{l}\text { IMP PHILIPPVS AVG } \\
\text { As previous. }\end{array}$ & $\begin{array}{l}\text { SAECVLARES AVGG } \\
\text { Low column inscribed COS III. }\end{array}$ & $\begin{array}{l}\text { RIC IV/ } \\
\text { III, } 24 \mathrm{c} .\end{array}$ & 248 & 6076 \\
\hline 138 & $\begin{array}{l}\text { ANT. } \\
2.93 \\
23.5 \\
1\end{array}$ & $\begin{array}{l}\text { IMP PHILIPPVS AVG } \\
\text { As previous.. }\end{array}$ & $\begin{array}{l}\text { SAECVLVM NOVVM } \\
\text { Hexastyle temple with statue (of } \\
\text { Rome) in centre of columns. }\end{array}$ & $\begin{array}{l}\text { RIC IV/ } \\
\text { III, } 25 \mathrm{~b} .\end{array}$ & 248 & 6042 \\
\hline 139 & $\begin{array}{l}\text { ANT. } \\
3.92 \\
23.5 \\
1\end{array}$ & $\begin{array}{l}\text { IMP M IVL PHILIPPVS } \\
\text { AVG } \\
\text { As previous. }\end{array}$ & $\begin{array}{l}\text { AEQVITAS AVGG } \\
\text { Aequitas stg 1., hld. scales and } \\
\text { cornucopiae. }\end{array}$ & $\begin{array}{l}\text { RIC IV/ } \\
\text { III, } 27 \mathrm{~b} .\end{array}$ & $244-247$ & 6039 \\
\hline 140 & $\begin{array}{l}\text { ANT. } \\
3.77 \\
23.2 \\
1\end{array}$ & $\begin{array}{l}\text { IMP M IVL PHILIPPVS } \\
\text { AVG } \\
\text { As previous. }\end{array}$ & $\begin{array}{l}\text { AEQVITAS AVGG } \\
\text { As previous. }\end{array}$ & $\begin{array}{l}\text { RIC IV/ } \\
\text { III, } 27 \mathrm{~b} .\end{array}$ & $244-247$ & 6038 \\
\hline 141 & $\begin{array}{l}\text { ANT. } \\
3.56 \\
22.0 \\
6\end{array}$ & $\begin{array}{l}\text { IMP M IVL PHILIPPVS } \\
\text { AVG } \\
\text { As previous. }\end{array}$ & $\begin{array}{l}\text { ANNONA AVGG } \\
\text { Annona stg 1., hld. corn ears over } \\
\text { modius and cornucopiae. }\end{array}$ & $\begin{array}{l}\text { RIC IV/ } \\
\text { III, 28c. }\end{array}$ & $244-247$ & 6060 \\
\hline 142 & $\begin{array}{l}\text { ANT. } \\
3.50 \\
23.7 \\
6\end{array}$ & $\begin{array}{l}\text { IMP M IVL PHILIPPVS } \\
\text { AVG } \\
\text { As previous. }\end{array}$ & $\begin{array}{l}\text { ANNONA AVGG } \\
\text { As previous. }\end{array}$ & $\begin{array}{l}\text { RIC IV/ } \\
\text { III, } 28 \mathrm{c} .\end{array}$ & $244-247$ & 6048 \\
\hline 143 & $\begin{array}{l}\text { ANT. } \\
4.11 \\
22.9 \\
12\end{array}$ & $\begin{array}{l}\text { IMP M IVL PHILIPPVS } \\
\text { AVG } \\
\text { As previous. }\end{array}$ & $\begin{array}{l}\text { ANNONA AVGG } \\
\text { Annona stg 1., hld. corn ears over } \\
\text { prow and cornucopiae. }\end{array}$ & $\begin{array}{l}\text { RIC IV/ } \\
\text { III, } 29 .\end{array}$ & $244-247$ & 6068 \\
\hline 144 & $\begin{array}{l}\text { ANT. } \\
3.55 \\
24.2 \\
7\end{array}$ & $\begin{array}{l}\text { IMP M IVL PHILIPPVS } \\
\text { AVG } \\
\text { As previous. }\end{array}$ & $\begin{array}{l}\text { ANNONA AVGG } \\
\text { As previous. }\end{array}$ & $\begin{array}{l}\text { RIC IV/ } \\
\text { III, } 29 .\end{array}$ & $244-247$ & 6045 \\
\hline
\end{tabular}




\begin{tabular}{|c|c|c|c|c|c|c|}
\hline 145 & $\begin{array}{l}\text { ANT. } \\
4.28 \\
22.2 \\
1\end{array}$ & $\begin{array}{l}\text { IMP M IVL PHILIPPVS } \\
\text { AVG } \\
\text { As previous. }\end{array}$ & $\begin{array}{l}\text { ANNONA AVGG } \\
\text { As previous. }\end{array}$ & $\begin{array}{l}\text { RIC IV/ } \\
\text { III, } 29 .\end{array}$ & $244-247$ & 6053 \\
\hline 146 & $\begin{array}{l}\text { ANT. } \\
3.05 \\
23.8 \\
11\end{array}$ & $\begin{array}{l}\text { IMP M IVL PHILIPPVS } \\
\text { AVG } \\
\text { As previous. }\end{array}$ & $\begin{array}{l}\text { ANNONA AVGG } \\
\text { As previous. }\end{array}$ & $\begin{array}{l}\text { RIC IV/ } \\
\text { III, } 29 .\end{array}$ & $244-247$ & 6072 \\
\hline 147 & $\begin{array}{l}\text { ANT. } \\
3.71 \\
23.9 \\
7\end{array}$ & $\begin{array}{l}\text { IMP M IVL PHILIPPVS } \\
\text { AVG } \\
\text { As previous. }\end{array}$ & $\begin{array}{l}\text { FELICITAS TEMP } \\
\text { Felicitas stg. 1., hld. long caduceus } \\
\text { and cornucopiae. }\end{array}$ & $\begin{array}{l}\text { RIC IV/ } \\
\text { III, } 31 .\end{array}$ & $244-247$ & 6077 \\
\hline 148 & $\begin{array}{l}\text { ANT. } \\
3.99 \\
23.4 \\
1\end{array}$ & $\begin{array}{l}\text { IMP M IVL PHILIPPVS } \\
\text { AVG } \\
\text { As previous. }\end{array}$ & $\begin{array}{l}\text { FIDES MILIT } \\
\text { Fides stg. } 1 \text {, hld. two standards. }\end{array}$ & $\begin{array}{l}\text { RIC IV/ } \\
\text { III, 32b. }\end{array}$ & $244-247$ & 6063 \\
\hline 149 & $\begin{array}{l}\text { ANT. } \\
4.21 \\
22.6 \\
1\end{array}$ & $\begin{array}{l}\text { IMP M IVL PHILIPPVS } \\
\text { AVG } \\
\text { As previous. }\end{array}$ & $\begin{array}{l}\text { LAETIT FVNDAT } \\
\text { Laetitia stg. 1., hld. wreath and rudder. }\end{array}$ & $\begin{array}{l}\text { RIC IV/ } \\
\text { III, 36b. }\end{array}$ & $244-247$ & 6037 \\
\hline 150 & $\begin{array}{l}\text { ANT. } \\
4.30 \\
22.0 \\
7\end{array}$ & $\begin{array}{l}\text { IMP M IVL PHILIPPVS } \\
\text { AVG } \\
\text { As previous. }\end{array}$ & $\begin{array}{l}\text { LAETIT FVNDAT } \\
\text { As previous. }\end{array}$ & $\begin{array}{l}\text { RIC IV/ } \\
\text { III, 36b. }\end{array}$ & $244-247$ & 6050 \\
\hline 151 & $\begin{array}{l}\text { ANT. } \\
4.57 \\
23.2 \\
7\end{array}$ & $\begin{array}{l}\text { IMP M IVL PHILIPPVS } \\
\text { AVG } \\
\text { Bust radiate, dr., cuiras- } \\
\text { sed, r. }\end{array}$ & $\begin{array}{l}\text { PAX AETERN } \\
\text { Pax running 1., hld. branch and } \\
\text { transverse scepter. }\end{array}$ & $\begin{array}{l}\text { RIC IV/ } \\
\text { III, 40b. }\end{array}$ & $244-247$ & 6049 \\
\hline 152 & $\begin{array}{l}\text { ANT. } \\
3.60 \\
22.0 \\
1\end{array}$ & $\begin{array}{l}\text { IMP M IVL PHILIPPVS } \\
\text { AVG } \\
\text { As previous. }\end{array}$ & $\begin{array}{l}\text { PAX AETERN } \\
\text { As previous. }\end{array}$ & $\begin{array}{l}\text { RIC IV/ } \\
\text { III, 40b. }\end{array}$ & $244-247$ & 6043 \\
\hline 153 & $\begin{array}{l}\text { ANT. } \\
2.81 \\
21.5 \\
12\end{array}$ & $\begin{array}{l}\text { IMP M IVL PHILIPPVS } \\
\text { AVG } \\
\text { As previous. }\end{array}$ & $\begin{array}{l}\text { [ROMA AE]TERNAE } \\
\text { Roma std. 1., hld. Victory and scepter; } \\
\text { at foot side, shield, }\end{array}$ & $\begin{array}{l}\text { RIC IV/ } \\
\text { III, 44b. }\end{array}$ & $244-247$ & 6044 \\
\hline 154 & $\begin{array}{l}\text { ANT. } \\
3.89 \\
20.4 \\
1\end{array}$ & $\begin{array}{l}\text { IMP M IVL PHILIPPVS } \\
\text { AVG } \\
\text { As previous. }\end{array}$ & $\begin{array}{l}\text { ROMA AETERNAE } \\
\text { As previous. }\end{array}$ & $\begin{array}{l}\text { RIC IV/ } \\
\text { III, 44b. }\end{array}$ & $244-247$ & 6036 \\
\hline 155 & $\begin{array}{l}\text { ANT. } \\
4.33 \\
24.5 \\
7\end{array}$ & $\begin{array}{l}\text { IMP M IVL PHILIPPVS } \\
\text { AVG } \\
\text { As previous. }\end{array}$ & $\begin{array}{l}\text { ROMA AETERNAE } \\
\text { As previous, but altar } 1 \text {. }\end{array}$ & $\begin{array}{l}\text { RIC IV/ } \\
\text { III, } 45 .\end{array}$ & $244-247$ & 6047 \\
\hline
\end{tabular}




\begin{tabular}{|c|c|c|c|c|c|c|}
\hline 156 & $\begin{array}{l}\text { ANT. } \\
3.70 \\
23.1 \\
1\end{array}$ & $\begin{array}{l}\text { IMP M IVL PHILIPPVS } \\
\text { AVG } \\
\text { As previous. }\end{array}$ & $\begin{array}{l}\text { SALVS AVG } \\
\text { Salus stg. 1., feding snake coiled } \\
\text { round altar and hld. rudder. }\end{array}$ & $\begin{array}{l}\text { RIC IV/ } \\
\text { III, } 47 .\end{array}$ & $244-247$ & 6061 \\
\hline 157 & $\begin{array}{l}\text { ANT. } \\
4.47 \\
22.7 \\
12\end{array}$ & $\begin{array}{l}\text { IMP M IVL PHILIPPVS } \\
\text { AVG } \\
\text { As previous. }\end{array}$ & $\begin{array}{l}\text { SECVRIT ORBIS } \\
\text { Securitas std. 1., hld. scepter and } \\
\text { propping head on } 1 . \text { hand. }\end{array}$ & $\begin{array}{l}\text { RIC IV/ } \\
\text { III, } 48 \mathrm{~b} .\end{array}$ & $244-247$ & 6052 \\
\hline 158 & $\begin{array}{l}\text { ANT. } \\
3.98 \\
24.2 \\
7\end{array}$ & $\begin{array}{l}\text { IMP M IVL PHILIPPVS } \\
\text { AVG } \\
\text { As previous. }\end{array}$ & $\begin{array}{l}\text { VICTORIA AVGG } \\
\text { Victory advancing r., hld. wreath and } \\
\text { palm. }\end{array}$ & $\begin{array}{l}\text { RIC IV/ } \\
\text { III, } 49 \mathrm{~b} .\end{array}$ & $244-247$ & 6055 \\
\hline 159 & $\begin{array}{l}\text { ANT. } \\
4.14 \\
22.6 \\
5\end{array}$ & $\begin{array}{l}\text { M IVL PHILIPPVS } \\
\text { AVG } \\
\text { As previous. }\end{array}$ & $\begin{array}{l}\text { VICTORIA AVG } \\
\text { Victory advancing 1., hld. wreath and } \\
\text { palm. }\end{array}$ & $\begin{array}{l}\text { RIC IV/ } \\
\text { III, } 50 .\end{array}$ & $244-247$ & 6056 \\
\hline 160 & $\begin{array}{l}\text { ANT. } \\
3.46 \\
22.4 \\
7\end{array}$ & $\begin{array}{l}\text { IMP M IVL PHILIPPVS } \\
\text { AVG } \\
\text { As previous. }\end{array}$ & $\begin{array}{l}\text { VICTORIA AVGG } \\
\text { Victory stg. 1., hld. wreath and palm. }\end{array}$ & $\begin{array}{l}\text { RIC IV/ } \\
\text { III, } 51\end{array}$ & $244-247$ & 6054 \\
\hline 161 & $\begin{array}{l}\text { ANT. } \\
3.72 \\
22.0 \\
7\end{array}$ & $\begin{array}{l}\text { IMP M IVL PHILIPPVS } \\
\text { AVG } \\
\text { As previous. }\end{array}$ & $\begin{array}{l}\text { VIRTVS AVG } \\
\text { Virtus std. } 1 \text {. on cuirass, hld. branch } \\
\text { and spear. }\end{array}$ & $\begin{array}{l}\text { RIC IV/ } \\
\text { III, } 53 .\end{array}$ & $244-247$ & 6058 \\
\hline 162 & $\begin{array}{l}\text { ANT. } \\
4.21 \\
22.5 \\
12\end{array}$ & $\begin{array}{l}\text { IMP PHILIPPVS AVG } \\
\text { As previous. }\end{array}$ & $\begin{array}{l}\text { NOBILI-TAS AVGG } \\
\text { Nobilitas stg. r., hld. scepter and globe; } 1 . \\
\text { in field, S. }\end{array}$ & $\begin{array}{l}\text { RIC IV/ } \\
\text { III, } 8 .\end{array}$ & $244-249$ & 6057 \\
\hline 163 & $\begin{array}{l}\text { ANT. } \\
4.65 \\
24.6 \\
7\end{array}$ & $\begin{array}{l}\text { IMP PHILIPPVS AVG } \\
\text { As previous. }\end{array}$ & $\begin{array}{l}\text { AEQVITAS AVGG } \\
\text { Aequitas stg 1., hld. scales and } \\
\text { cornucopia. }\end{array}$ & $\begin{array}{l}\text { RIC IV/ } \\
\text { III, } 57 .\end{array}$ & $247-249$ & 6040 \\
\hline 164 & $\begin{array}{l}\text { ANT. } \\
3.79 \\
22.0 \\
7\end{array}$ & $\begin{array}{l}\text { IMP PHILIPPVS AVG } \\
\text { As previous. }\end{array}$ & $\begin{array}{l}\text { AEQVITAS AVGG } \\
\text { As previous. }\end{array}$ & $\begin{array}{l}\text { RIC IV/ } \\
\text { III, } 57 .\end{array}$ & $247-249$ & 6067 \\
\hline 165 & $\begin{array}{l}\text { ANT. } \\
4.32 \\
24.5 \\
1\end{array}$ & $\begin{array}{l}\text { IMP PHILIPPVS AVG } \\
\text { As previous. }\end{array}$ & $\begin{array}{l}\text { AETERNITAS AVGG } \\
\text { Elephant, quided by driver with goad } \\
\text { and wand, walking } 1 \text {. }\end{array}$ & $\begin{array}{l}\text { RIC IV/ } \\
\text { III, } 58 .\end{array}$ & $247-249$ & 6075 \\
\hline 166 & $\begin{array}{l}\text { ANT. } \\
3.21 \\
23.8 \\
12\end{array}$ & $\begin{array}{l}\text { IMP PHILIPPVS AVG } \\
\text { As previous. }\end{array}$ & $\begin{array}{l}\text { AETERNITAS AVGG } \\
\text { As previous. }\end{array}$ & $\begin{array}{l}\text { RIC IV/ } \\
\text { III, } 58 .\end{array}$ & $247-249$ & 6069 \\
\hline
\end{tabular}




\begin{tabular}{|l|l|l|}
167 & ANT. & IMP PHILIPPVS AVG \\
& 2.87 & As previous. \\
& 23.8 & \\
& 7 & \\
& & \\
& ANT. & IMP PHILIPPVS AVG \\
& 4.32 & As previous. \\
& 24.6 & \\
& 12 & \\
& & \\
& ANT. & IMP PHILIPPVS AVG \\
& 3.40 & As previous. \\
& 22.3 & \\
& 1 &
\end{tabular}

\begin{tabular}{|l|l|l|}
170 & ANT. & IMP C M IVL \\
& 3.78 & PHILIPPVS P F AVG \\
& 21.0 & PM \\
& 7 & As previous. \\
& & \\
& ANT. & IMP M IVL PHILIPPVS \\
& 3.87 & AVG \\
& 22.4 & As previous. \\
& 1 & \\
& ANT. & \\
& 3.69 & IMP PHILIPPVS AVG \\
& 21.7 & \\
& 7 &
\end{tabular}

ANNONA AVGG

Anonna stg. 1., folding corn-ears over prow and cornucopiae.

FIDES EXERCITVS

Four standards-the second from r., a legionary eagle.

ROMA AETERNAE

Roma std. 1., hld. Victory and spear; at her side, shield; in front altar.

(cat. 170-172)

\section{Mint of Antioch}

\section{SPES FELICITATIS [ORBIS] \\ Spes advancing 1., hld. flower and raising skirt. \\ SAECVLVM NOVVM \\ Hexastyle temple with statue (of \\ Rome) in centre of columms. \\ P M TR P IIII COS II P P \\ Felicitas stg. 1., hld. long caduceus and cornucopiae.}

\section{OTACILIA SEVERA}

(striking under Philip I)

(cat. 173-182)

\section{Mint of Rome}

\section{PVDICITIA AVG}

Pudicitia, veiled, std. 1., with hand drawing veil, transverse scepter in 1 .

\section{CONCORDIA AVGG}

Concordia std. 1., hld. patera and double cornucopiae.

\section{CONCORDIA AVGG}

As previous, but with single cornucopiae, to 1 . altar?.

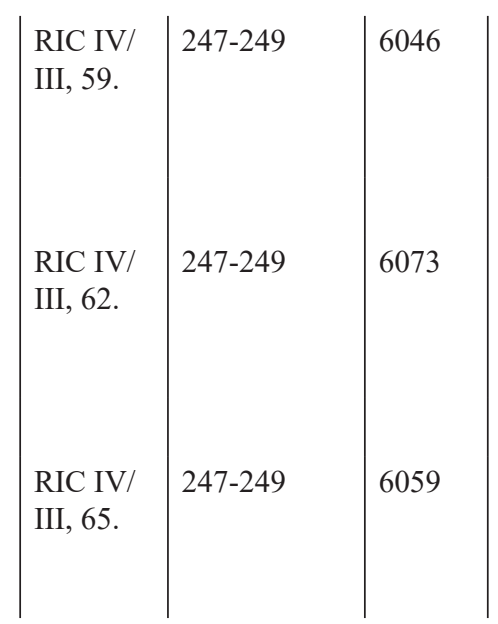

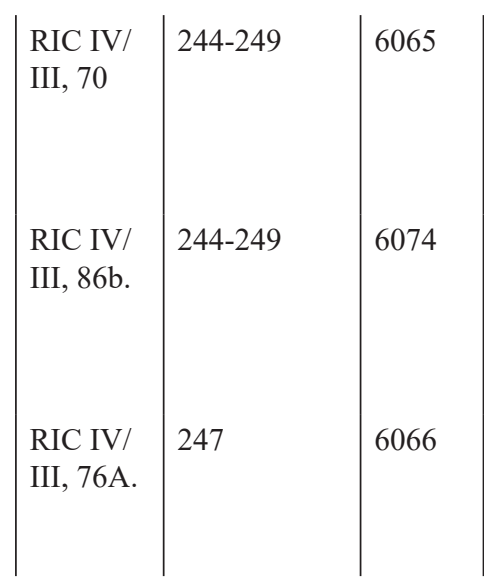

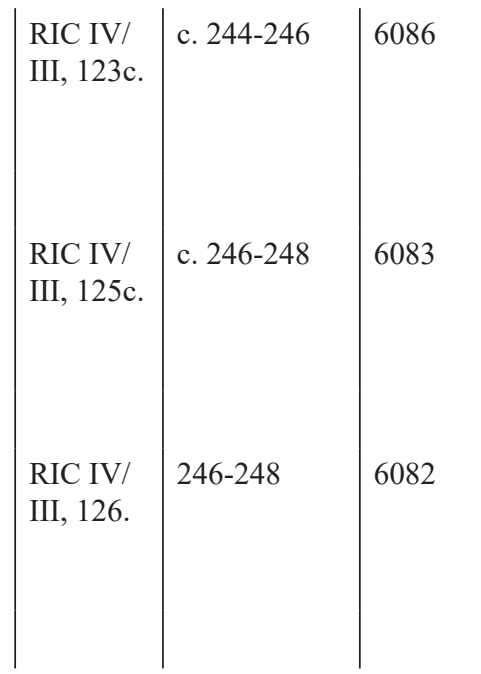




\begin{tabular}{|c|c|c|}
\hline 176 & $\begin{array}{l}\text { ANT. } \\
3.51 \\
22.9 \\
6\end{array}$ & $\begin{array}{l}\text { M OTACIL SEVERA } \\
\text { AVG } \\
\text { As previous. }\end{array}$ \\
\hline 177 & $\begin{array}{l}\text { ANT. } \\
3.78 \\
24.3 \\
7\end{array}$ & $\begin{array}{l}\text { M OTACIL SEVERA } \\
\text { AVG } \\
\text { As previous. }\end{array}$ \\
\hline 178 & $\begin{array}{l}\text { ANT. } \\
4.12 \\
22.0 \\
1\end{array}$ & $\begin{array}{l}\text { M OTACIL SEVERA } \\
\text { AVG } \\
\text { As previous. }\end{array}$ \\
\hline 179 & $\begin{array}{l}\text { ANT. } \\
3.44 \\
22.0 \\
1\end{array}$ & $\begin{array}{l}\text { OTACIL SEVERA AVG } \\
\text { As previous. }\end{array}$ \\
\hline 180 & $\begin{array}{l}\text { ANT. } \\
4.04 \\
22.1 \\
6\end{array}$ & $\begin{array}{l}\text { OTACIL SEVERA AVG } \\
\text { Bust, diademed, dr., on } \\
\text { cresent, r. }\end{array}$ \\
\hline 181 & $\begin{array}{l}\text { ANT. } \\
3.95 \\
23.2 \\
7\end{array}$ & $\begin{array}{l}\text { OTACIL SEVERA AVG } \\
\text { As previous. }\end{array}$ \\
\hline 182 & $\begin{array}{l}\text { ANT. } \\
4.01 \\
24.2 \\
6\end{array}$ & $\begin{array}{l}\text { OTACIL SEVERA AVG } \\
\text { As previous. }\end{array}$ \\
\hline
\end{tabular}

CONCORDIA AVGG
As previous.
CONCORDIA AVGG
As previous.
IVNO CONSERVAT
Juno, veiled, stg. 1., hld. patera and
scepter.
PIETAS AVGVSTAE
Pietas stg. 1., raising r. hand and hld.
box of perfume.
CONCORDIA AVGG
Concordia std. 1., hld. patera and
cornucopiae, to 1., altar.
PIETAS AVGG
Pietas stg. 1., raising r. hand over altar
and hld. box of perfume. In field $1 ., \Delta$.
As previous.
PIES AVGG

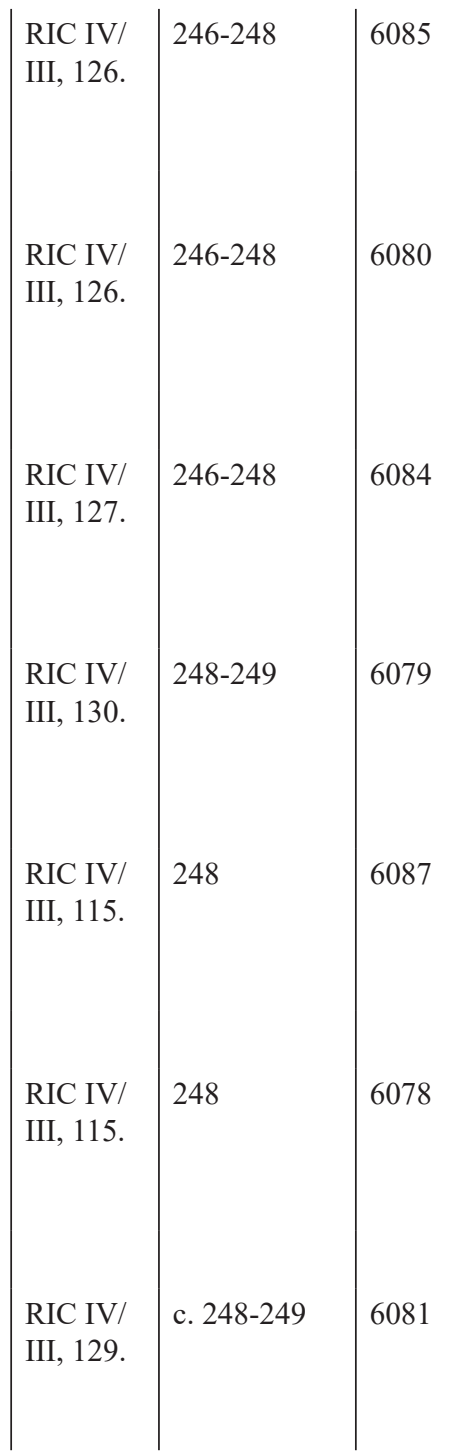

PHILIP II

(cat. 183-189)

\section{Mint of Rome}

\section{(As Caesar)}

\begin{tabular}{|l|l|l}
183 & ANT. & M IVL PHILIPPVS \\
& 3.84 & CAES \\
& 23.7 & Bust radiate, dr., cuir., r. \\
& 7 & \\
& & \\
& & \\
& ANT. & M IVL PHILIPPVS \\
& 3.80 & CAES \\
& 22.0 & As previous. \\
& 12 & \\
& & \\
& ANT. & M IVL PHILIPPVS \\
& 4.27 & CAES \\
& 23.0 & As previous. \\
& 7 &
\end{tabular}

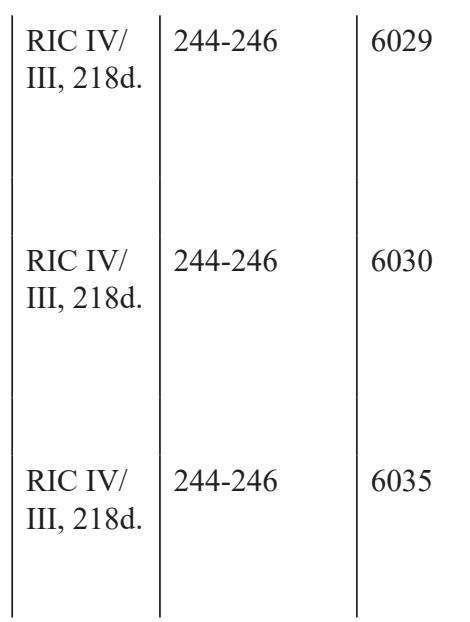




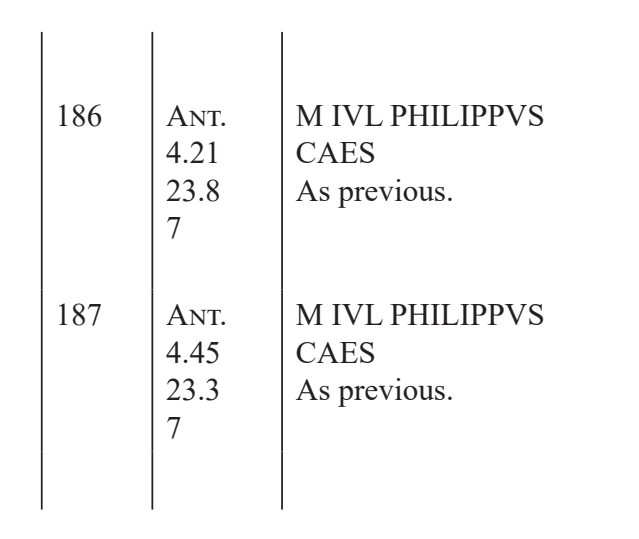

\section{PRINCIPI I-VVENT}

As previous.

PRINCIPI IVVENT

As previous, but cuptive at feat of

Philip II, 1.

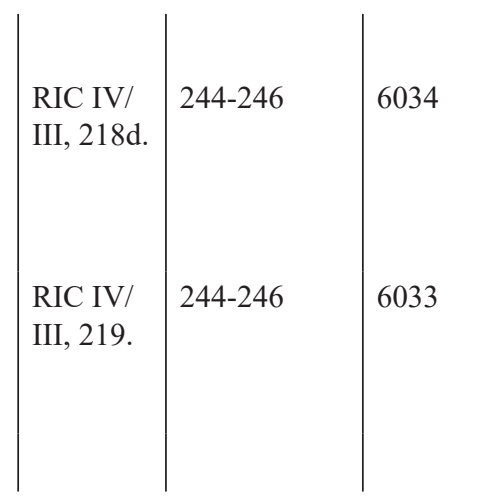

\section{(As Augustus)}

\begin{tabular}{|l|l|l|}
188 & ANT. & IMP M IVL PHILIPPVS \\
& 3.67 & AVG \\
& 22.1 & As previous. \\
& 1 & \\
& & \\
& ANT. & IMP PHILIPPVS AVG \\
& 3.68 & As previous. \\
& 24.5 & \\
& 7 &
\end{tabular}

PAX AETERNA
Pax stg. 1., hld. branch and transverse
sceptre.
PAX AETERNA
Pax running 1., hld. branch and
transverse scepter.

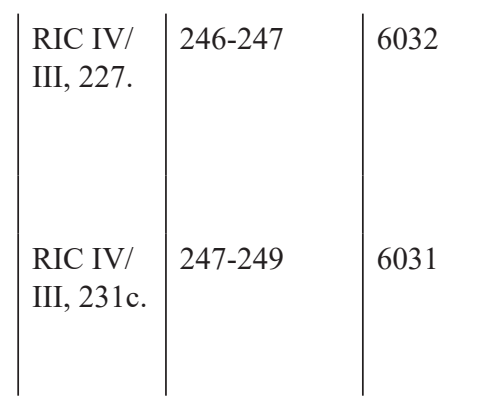

\section{TRAJAN DECIUS \\ (cat. 190-221) \\ Mint of Rome}

\begin{tabular}{|c|c|c|}
\hline 190 & $\begin{array}{l}\text { AnT. } \\
4.09 \\
23.9 \\
1\end{array}$ & $\begin{array}{l}\text { IMP TRAIANVS } \\
\text { DECIVS AVG } \\
\text { Bust, radiate, dr., } \\
\text { cuirassed, r. }\end{array}$ \\
\hline 191 & $\begin{array}{l}\text { ANT. } \\
4.18 \\
24.5 \\
12\end{array}$ & $\begin{array}{l}\text { IMP C M Q TRAIANVS } \\
\text { DECIVS AVG } \\
\text { As previous. }\end{array}$ \\
\hline 192 & $\begin{array}{l}\text { ANT. } \\
4.13 \\
22.0 \\
12\end{array}$ & $\begin{array}{l}\text { IMP C M Q TRAIANVS } \\
\text { DECIVS AVG } \\
\text { As previous. }\end{array}$ \\
\hline 193 & $\begin{array}{l}\text { ANT. } \\
3.12 \\
22.1 \\
1\end{array}$ & $\begin{array}{l}\text { IMP C M Q TRAIANVS } \\
\text { DECIVS AVG } \\
\text { As previous. }\end{array}$ \\
\hline 194 & $\begin{array}{l}\text { AnT. } \\
3.98 \\
24.1 \\
1\end{array}$ & $\begin{array}{l}\text { IMP C M Q TRAIANVS } \\
\text { DECIVS AVG } \\
\text { As previous. }\end{array}$ \\
\hline
\end{tabular}

PANNONIAE

Pannonia, veiled, stg. front, head r., raising $r$. hand and hld. standard.

\section{ABVNDATIA AVG}

Abundantia stg. r., emptying

cornucopiae held in both hands.

\section{ABVNDATIA AVG}

As previous.

\section{ABVNDATIA AVG}

As previous

ABVNDATIA AVG

As previous.

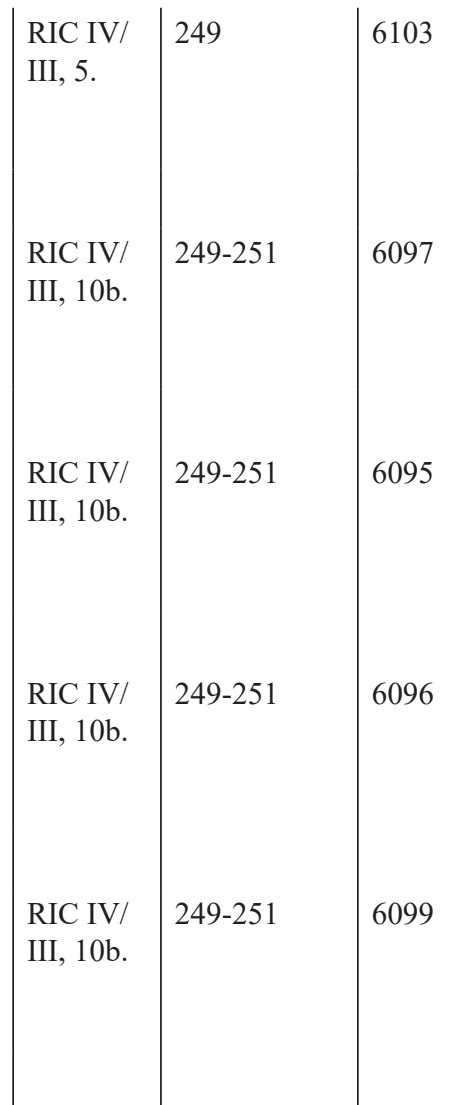




\begin{tabular}{|c|c|c|c|c|c|c|}
\hline 195 & $\begin{array}{l}\text { ANT. } \\
3.87 \\
21.9 \\
7\end{array}$ & $\begin{array}{l}\text { IMP C M Q TRAIANVS } \\
\text { DECIVS AVG } \\
\text { As previous. }\end{array}$ & $\begin{array}{l}\text { ABVNDATIA AVG } \\
\text { As previous. }\end{array}$ & $\begin{array}{l}\text { RIC IV/ } \\
\text { III, } 10 b \text {. }\end{array}$ & $249-251$ & 6098 \\
\hline 196 & $\begin{array}{l}\text { ANT. } \\
3.51 \\
20.9 \\
12\end{array}$ & $\begin{array}{l}\text { IMP C M Q TRAIANVS } \\
\text { DECIVS AVG } \\
\text { As previous. }\end{array}$ & $\begin{array}{l}\text { ADVENTVS AVG } \\
\text { Trajan Decius on horse pacing l., } \\
\text { raising r. hand and hld. short scepter. }\end{array}$ & $\begin{array}{l}\text { RIC IV/ } \\
\text { III, } 11 \mathrm{~b} .\end{array}$ & $249-251$ & 6090 \\
\hline 197 & $\begin{array}{l}\text { ANT. } \\
3.14 \\
22.5 \\
1\end{array}$ & $\begin{array}{l}\text { IMP C M Q TRAIANVS } \\
\text { DECIVS AVG } \\
\text { As previous. }\end{array}$ & $\begin{array}{l}\text { ADVENTVS AVG } \\
\text { As previous. }\end{array}$ & $\begin{array}{l}\text { RIC IV/ } \\
\text { III, } 11 \mathrm{~b} .\end{array}$ & $249-251$ & 6092 \\
\hline 198 & $\begin{array}{l}\text { ANT. } \\
3.32 \\
23.9 \\
7\end{array}$ & $\begin{array}{l}\text { IMP C M Q TRAIANVS } \\
\text { DECIVS AVG } \\
\text { As previous. }\end{array}$ & $\begin{array}{l}\text { ADVENTVS AVG } \\
\text { As previous. }\end{array}$ & $\begin{array}{l}\text { RIC IV/ } \\
\text { III, } 11 \mathrm{~b} .\end{array}$ & $249-251$ & 6120 \\
\hline 199 & $\begin{array}{l}\text { ANT. } \\
3.55 \\
21.7 \\
1\end{array}$ & $\begin{array}{l}\text { IMP C M Q TRAIANVS } \\
\text { DECIVS AVG } \\
\text { As previous. }\end{array}$ & $\begin{array}{l}\text { DACIA } \\
\text { Dacia, wearing robe reaching feet, stg. 1., } \\
\text { hld. vertical staff with ass's head. }\end{array}$ & $\begin{array}{l}\text { RIC IV/ } \\
\text { III, } 12 \mathrm{~b} .\end{array}$ & $249-251$ & 6108 \\
\hline 200 & $\begin{array}{l}\text { ANT. } \\
3.51 \\
21.9 \\
7\end{array}$ & $\begin{array}{l}\text { IMP C M Q TRAIANVS } \\
\text { DECIVS AVG } \\
\text { As previous. }\end{array}$ & $\begin{array}{l}\text { DACIA } \\
\text { As previous. }\end{array}$ & $\begin{array}{l}\text { RIC IV/ } \\
\text { III, 12b. }\end{array}$ & $249-251$ & 6115 \\
\hline 201 & $\begin{array}{l}\text { ANT. } \\
4.13 \\
22.6 \\
6\end{array}$ & $\begin{array}{l}\text { IMP C M Q TRAIANVS } \\
\text { DECIVS AVG } \\
\text { As previous. }\end{array}$ & $\begin{array}{l}\text { DACIA } \\
\text { As previous. }\end{array}$ & $\begin{array}{l}\text { RIC IV/ } \\
\text { III, 12b. }\end{array}$ & $249-251$ & 6119 \\
\hline 202 & $\begin{array}{l}\text { ANT. } \\
4.65 \\
21.0 \\
7\end{array}$ & $\begin{array}{l}\text { IMP C M Q TRAIANVS } \\
\text { DECIVS AVG } \\
\text { As previous. }\end{array}$ & $\begin{array}{l}\text { DACIA } \\
\text { As previous. }\end{array}$ & $\begin{array}{l}\text { RIC IV/ } \\
\text { III, } 12 b .\end{array}$ & $249-251$ & 6118 \\
\hline 203 & $\begin{array}{l}\text { ANT. } \\
3.27 \\
21.4 \\
7\end{array}$ & $\begin{array}{l}\text { IMP C M Q TRAIANVS } \\
\text { DECIVS AVG } \\
\text { As previous. }\end{array}$ & $\begin{array}{l}\text { DACIA } \\
\text { As previous. }\end{array}$ & $\begin{array}{l}\text { RIC IV/ } \\
\text { III, } 12 b .\end{array}$ & $249-251$ & 6117 \\
\hline 204 & $\begin{array}{l}\text { ANT. } \\
3.77 \\
22.4 \\
1\end{array}$ & $\begin{array}{l}\text { IMP C M Q TRAIANVS } \\
\text { DECIVS AVG } \\
\text { As previous. }\end{array}$ & $\begin{array}{l}\text { DACIA } \\
\text { As previous. }\end{array}$ & $\begin{array}{l}\text { RIC IV/ } \\
\text { III, } 12 \mathrm{~b} .\end{array}$ & $249-251$ & 6106 \\
\hline 205 & $\begin{array}{l}\text { ANT. } \\
3.81 \\
20.0 \\
7\end{array}$ & $\begin{array}{l}\text { IMP C M Q TRAIANVS } \\
\text { DECIVS AVG } \\
\text { As previous. }\end{array}$ & $\begin{array}{l}\text { GENIVS EXERC ILLVRICIANI } \\
\text { Genius wearing polos on head, stg. } \\
\text { 1., hld. patera and cornucopiae; to r., } \\
\text { standard. }\end{array}$ & $\begin{array}{l}\text { RIC IV/ } \\
\text { III, 16c. }\end{array}$ & $249-251$ & 6094 \\
\hline
\end{tabular}




\begin{tabular}{|c|c|c|c|c|c|c|}
\hline 206 & $\begin{array}{l}\text { ANT. } \\
4.02 \\
20.6 \\
7\end{array}$ & $\begin{array}{l}\text { IMP C M Q TRAIANVS } \\
\text { DECIVS AVG } \\
\text { As previous. }\end{array}$ & $\begin{array}{l}\text { GENIVS EXERC ILLVRICIANI } \\
\text { As previous. }\end{array}$ & $\begin{array}{l}\text { RIC IV/ } \\
\text { III, 16c. }\end{array}$ & $249-251$ & 6088 \\
\hline 207 & $\begin{array}{l}\text { ANT. } \\
3.40 \\
23.4 \\
7\end{array}$ & $\begin{array}{l}\text { IMP C M Q TRAIANVS } \\
\text { DECIVS AVG } \\
\text { As previous. }\end{array}$ & $\begin{array}{l}\text { GENIVS EXERC ILLVRICIANI } \\
\text { As previous. }\end{array}$ & $\begin{array}{l}\text { RIC IV/ } \\
\text { III, } 16 \mathrm{c} .\end{array}$ & $249-251$ & 6093 \\
\hline 208 & $\begin{array}{l}\text { ANT. } \\
3.39 \\
21.4 \\
5\end{array}$ & $\begin{array}{l}\text { IMP C M Q TRAIANVS } \\
\text { DECIVS AVG } \\
\text { As previous. }\end{array}$ & $\begin{array}{l}\text { GENIVS EXERC ILLVRICIANI } \\
\text { As previous. }\end{array}$ & $\begin{array}{l}\text { RIC IV/ } \\
\text { III, } 16 \mathrm{c} .\end{array}$ & $249-251$ & 6091 \\
\hline 209 & $\begin{array}{l}\text { ANT. } \\
4.37 \\
22.6 \\
7\end{array}$ & $\begin{array}{l}\text { IMP C M Q TRAIANVS } \\
\text { DECIVS AVG } \\
\text { As previous. }\end{array}$ & $\begin{array}{l}\text { PANNONIAE } \\
\text { The two Pannoniae, veiled, stg. front } \\
\text { turning r. and } 1 \text {. away from one another, } \\
\text { each wears robe reaching to feet and } \\
\text { holds standards. }\end{array}$ & $\begin{array}{l}\text { RIC IV/ } \\
\text { III, } 21 \mathrm{~b} .\end{array}$ & $249-251$ & 6100 \\
\hline 210 & $\begin{array}{l}\text { ANT. } \\
3.51 \\
21.7 \\
1\end{array}$ & $\begin{array}{l}\text { IMP C M Q TRAIANVS } \\
\text { DECIVS AVG } \\
\text { As previous. }\end{array}$ & $\begin{array}{l}\text { PANNONIAE } \\
\text { As previous. }\end{array}$ & $\begin{array}{l}\text { RIC IV/ } \\
\text { III, } 21 \mathrm{~b} .\end{array}$ & $249-251$ & 6110 \\
\hline 211 & $\begin{array}{l}\text { ANT. } \\
4.45 \\
23.0 \\
12\end{array}$ & $\begin{array}{l}\text { IMP C M Q TRAIANVS } \\
\text { DECIVS AVG } \\
\text { As previous. }\end{array}$ & $\begin{array}{l}\text { PANNONIAE } \\
\text { As previous. }\end{array}$ & $\begin{array}{l}\text { RIC IV/ } \\
\text { III, } 21 \mathrm{~b} .\end{array}$ & $249-251$ & 6101 \\
\hline 212 & $\begin{array}{l}\text { ANT. } \\
3.30 \\
24.0 \\
7\end{array}$ & $\begin{array}{l}\text { IMP C M Q TRAIANVS } \\
\text { DECIVS AVG } \\
\text { As previous. }\end{array}$ & $\begin{array}{l}\text { PANNONIAE } \\
\text { As previous. }\end{array}$ & $\begin{array}{l}\text { RIC IV/ } \\
\text { III, } 21 \mathrm{~b} .\end{array}$ & $249-251$ & 6107 \\
\hline 213 & $\begin{array}{l}\text { ANT. } \\
3.98 \\
21.6 \\
1\end{array}$ & $\begin{array}{l}\text { IMP C M Q TRAIANVS } \\
\text { DECIVS AVG } \\
\text { As previous. }\end{array}$ & $\begin{array}{l}\text { VBERITAS AVG } \\
\text { Uberitas stg. 1., hld. purse and } \\
\text { cornucopiae. }\end{array}$ & $\begin{array}{l}\text { RIC IV/ } \\
\text { III, 28b. }\end{array}$ & $249-251$ & 6112 \\
\hline 214 & $\begin{array}{l}\text { ANT. } \\
3.53 \\
21.7 \\
11\end{array}$ & $\begin{array}{l}\text { IMP C M Q TRAIANVS } \\
\text { DECIVS AVG } \\
\text { As previous. }\end{array}$ & $\begin{array}{l}\text { VBERITAS AVG } \\
\text { As previous. }\end{array}$ & $\begin{array}{l}\text { RIC IV/ } \\
\text { III, 28b. }\end{array}$ & $249-251$ & 6104 \\
\hline 215 & $\begin{array}{l}\text { ANT. } \\
3.87 \\
22.4 \\
7\end{array}$ & $\begin{array}{l}\text { IMP C M Q TRAIANVS } \\
\text { DECIVS AVG } \\
\text { As previous. }\end{array}$ & $\begin{array}{l}\text { VBERITAS AVG } \\
\text { As previous. }\end{array}$ & $\begin{array}{l}\text { RIC IV/ } \\
\text { III, } 28 b .\end{array}$ & $249-251$ & 6109 \\
\hline 216 & $\begin{array}{l}\text { ANT. } \\
3.48 \\
22.5 \\
6\end{array}$ & $\begin{array}{l}\text { IMP C M Q TRAIANVS } \\
\text { DECIVS AVG } \\
\text { As previous. }\end{array}$ & $\begin{array}{l}\text { VBERITAS AVG } \\
\text { As previous. }\end{array}$ & $\begin{array}{l}\text { RIC IV/ } \\
\text { III, 28b. }\end{array}$ & $249-251$ & 6111 \\
\hline
\end{tabular}




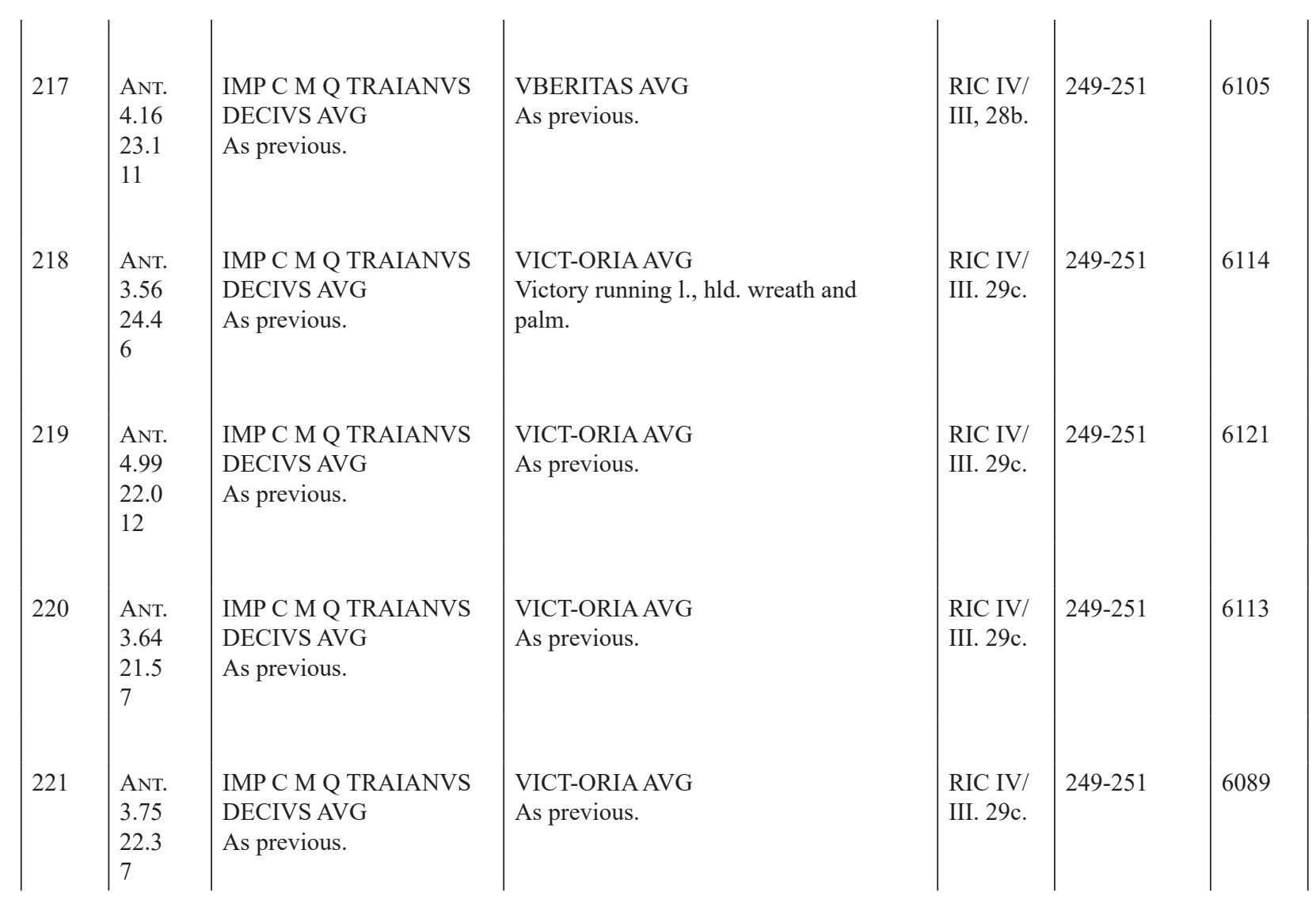

(cat 222-223)

\section{Mint of Milan}

\begin{tabular}{|l|l|l}
222 & ANT. & IMP CAE TRA DEC \\
& 2.55 & AVG \\
& 21.4 & As previous. \\
& 7 & \\
& & \\
223 & ANT. & IMP CAE TRA DEC \\
& 2.79 & AVG \\
& 23.9 & As previous. \\
& 7 &
\end{tabular}

DACIA FELIX

Dacia stg. 1., hld. standard.

\section{PANNONIAE}

The two Pannoniae, veiled, stg. r. and l., facing one another, clasping $r$. hands in front of standard in centre.

HERENNIA ETRUSCILLA (Striking under Trajan Decius) (cat. 224-226)

\section{Mint of Rome}

\section{FECVNDITAS AVG}

Fecunditas stg. 1., hld. r. hand over child stg. r., with hands raised, and cornucopiae in 1 . hand.

\section{PVDICITIA AVG}

Pudicitia veiled, stg. 1., with $\mathrm{r}$. hand drawing veil and hld. scepter.

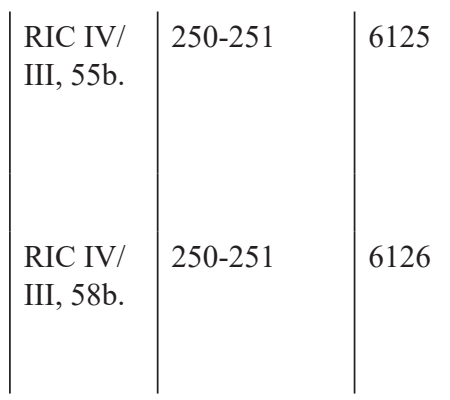




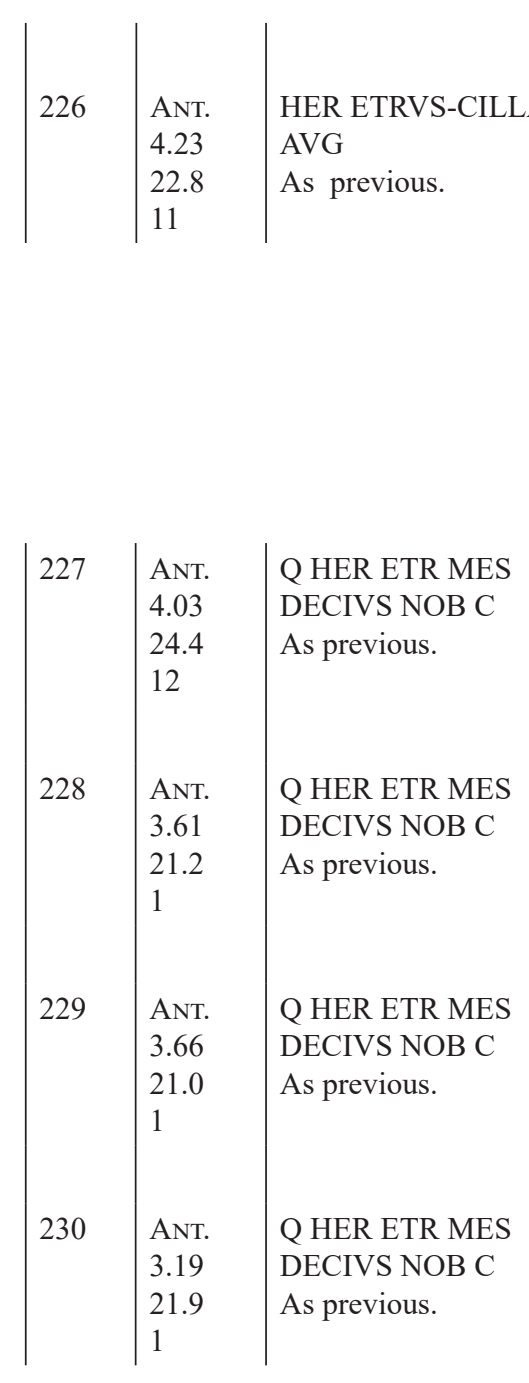

\section{PVDICITIA AVG}

Pudicitia veiled, std. 1., with $\mathrm{r}$. hand

drawing veil and hld. scepter.

\section{HERENNIUS ETRUSCUS}

(cat. 227-230)

\section{Mint of Rome}

(As Caesar)

\section{PIETAS A-VGG \\ Mercury stg. 1., hld. purse and caduceus. \\ PIETAS AVGVSTORVM \\ Sprinkler, simpulum, jug and lituus. \\ PRINCIPI IVVENTVTIS \\ Apollo std. 1., hld. branch and resting \\ elbow on lyre. \\ PRINCIPI IVVENTVTIS \\ Herennius, in military dress, stg.l., hld. \\ wand and transverse spear. \\ HOSTILIAN (Caesar) \\ (cat 231-232) \\ Mint of Rome}

C VALENS HOSTIL MES QVINTVS N C Bust, radiate, dr., r.

C VALENS HOSTIL MES QVINTVS N C Bust, radiate, dr., r.
MARTI PROPVGNATORI

Mars advancing r., hld. spear and shield.

PRINCIPI AVVENTVTIS

Hostilian stg. 1.,hld. standard and spear reversed.

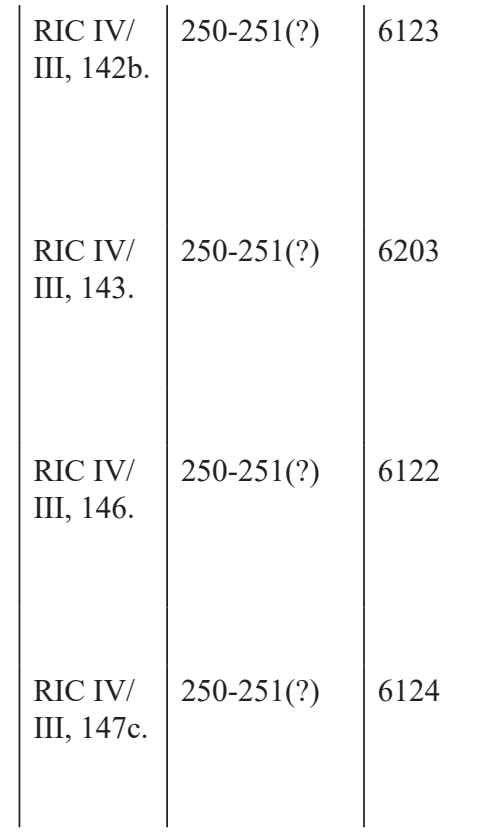

\section{TREBONIANUS GALLUS (cat 233-254) \\ Mint of Rome}

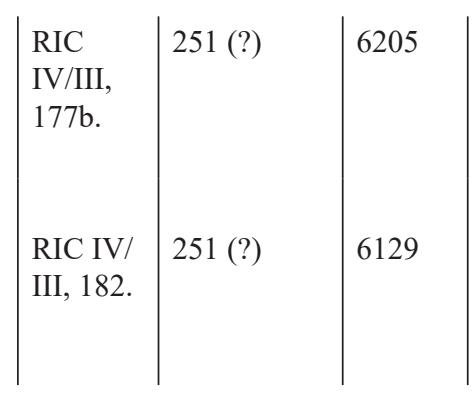

\section{AETERNITAS AVGG \\ Aeternitas stg. 1., hld. phoenix on globe, 1 . hand, raising skirt, at side.}

RIC IV/

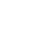




\begin{tabular}{|c|c|c|c|c|c|c|}
\hline 234 & $\begin{array}{l}\text { ANT. } \\
1.82 \\
22.0 \\
1\end{array}$ & $\begin{array}{l}\text { IMP CAE C VIB TREB } \\
\text { GALLVS AVG } \\
\text { As previous. }\end{array}$ & $\begin{array}{l}\text { AETERNITAS AVGG } \\
\text { As previous. }\end{array}$ & $\begin{array}{l}\text { RIC IV/ } \\
\text { III, } 30 .\end{array}$ & $251-253$ & 6159 \\
\hline 235 & $\begin{array}{l}\text { ANT. } \\
3.43 \\
21.6 \\
1\end{array}$ & $\begin{array}{l}\text { IMP CAE C VIB TREB } \\
\text { GALLVS AVG } \\
\text { As previous. }\end{array}$ & $\begin{array}{l}\text { ANNONA AVGG } \\
\text { Annona stg. r., hld. rudder upright and corn- } \\
\text { ears, 1. foot on prow. }\end{array}$ & $\begin{array}{l}\text { RIC IV/ } \\
\text { III, } 31\end{array}$ & $251-253$ & 6148 \\
\hline 236 & $\begin{array}{l}\text { ANT. } \\
3.07 \\
23.9 \\
7\end{array}$ & $\begin{array}{l}\text { IMP CAE C VIB TREB } \\
\text { GALLVS AVG } \\
\text { As previous. }\end{array}$ & $\begin{array}{l}\text { ANNONA AVGG } \\
\text { As previous. }\end{array}$ & $\begin{array}{l}\text { RIC IV/ } \\
\text { III, } 31\end{array}$ & $251-253$ & 6137 \\
\hline 237 & $\begin{array}{l}\text { ANT. } \\
4.08 \\
23.7 \\
1\end{array}$ & $\begin{array}{l}\text { IMP CAE C VIB TREB } \\
\text { GALLVS AVG } \\
\text { As previous. }\end{array}$ & $\begin{array}{l}\text { ANNONA AVGG } \\
\text { As previous. }\end{array}$ & $\begin{array}{l}\text { RIC IV/ } \\
\text { III, } 31\end{array}$ & $251-253$ & 6163 \\
\hline 238 & $\begin{array}{l}\text { ANT. } \\
3.22 \\
22.3 \\
1\end{array}$ & $\begin{array}{l}\text { IMP CAE C VIB TREB } \\
\text { GALLVS AVG } \\
\text { As previous. }\end{array}$ & $\begin{array}{l}\text { ANNONA AVGG } \\
\text { As previous. }\end{array}$ & $\begin{array}{l}\text { RIC IV/ } \\
\text { III, } 31\end{array}$ & $251-253$ & 6149 \\
\hline 239 & $\begin{array}{l}\text { ANT. } \\
2.69 \\
20.0 \\
11\end{array}$ & $\begin{array}{l}\text { IMP CAE C VIB TREB } \\
\text { GALLVS AVG } \\
\text { As previous. }\end{array}$ & $\begin{array}{l}\text { ANNONA AVGG } \\
\text { As previous. }\end{array}$ & $\begin{array}{l}\text { RIC IV/ } \\
\text { III, } 31\end{array}$ & $251-253$ & 6164 \\
\hline 240 & $\begin{array}{l}\text { ANT. } \\
2.82 \\
21.7 \\
12\end{array}$ & $\begin{array}{l}\text { IMP CAE C VIB TREB } \\
\text { GALLVS AVG } \\
\text { As previous. }\end{array}$ & $\begin{array}{l}\text { APOLL SALVTARI } \\
\text { Apollo stg. 1., hld. branch and lyre set on } \\
\text { rock. }\end{array}$ & $\begin{array}{l}\text { RIC IV/ } \\
\text { III, } 32 .\end{array}$ & $251-253$ & 6139 \\
\hline 241 & $\begin{array}{l}\text { ANT. } \\
2.60 \\
21.8 \\
5\end{array}$ & $\begin{array}{l}\text { IMP CAE C VIB TREB } \\
\text { GALLVS AVG } \\
\text { As previous. }\end{array}$ & $\begin{array}{l}\text { APOLL SALVTARI } \\
\text { As previous. }\end{array}$ & $\begin{array}{l}\text { RIC IV/ } \\
\text { III, } 32 .\end{array}$ & $251-253$ & 6138 \\
\hline 242 & $\begin{array}{l}\text { ANT. } \\
3.33 \\
21.8 \\
7\end{array}$ & $\begin{array}{l}\text { IMP CAE C VIB TREB } \\
\text { GALLVS AVG } \\
\text { As previous. }\end{array}$ & $\begin{array}{l}\text { FELICI-TAS PVBLICA } \\
\text { Felicitas stg. 1., hld. caduceus and cornuco- } \\
\text { piae, leans on column. }\end{array}$ & $\begin{array}{l}\text { RIC IV/ } \\
\text { III, 34A. }\end{array}$ & $251-253$ & 6145 \\
\hline 243 & $\begin{array}{l}\text { ANT. } \\
4.51 \\
23.4 \\
12\end{array}$ & $\begin{array}{l}\text { IMP CAE C VIB TREB } \\
\text { GALLVS AVG } \\
\text { As previous. }\end{array}$ & $\begin{array}{l}\text { FELICI-TAS PVBLICA } \\
\text { As previous. }\end{array}$ & $\begin{array}{l}\text { RIC IV/ } \\
\text { III, 34A. }\end{array}$ & $251-253$ & 6136 \\
\hline 244 & $\begin{array}{l}\text { ANT. } \\
3.50 \\
23.8 \\
7\end{array}$ & $\begin{array}{l}\text { IMP CAE C VIB TREB } \\
\text { GALLVS AVG } \\
\text { As previous. }\end{array}$ & $\begin{array}{l}\text { FELICI-TAS PVBLICA } \\
\text { As previous. }\end{array}$ & $\begin{array}{l}\text { RIC IV/ } \\
\text { III, 34A. }\end{array}$ & $251-253$ & 6146 \\
\hline
\end{tabular}




\begin{tabular}{|c|c|c|c|c|c|c|}
\hline 245 & $\begin{array}{l}\text { ANT. } \\
3.45 \\
22.3 \\
12\end{array}$ & $\begin{array}{l}\text { IMP CAE C VIB TREB } \\
\text { GALLVS AVG } \\
\text { As previous. }\end{array}$ & $\begin{array}{l}\text { FELICI-TAS PVBLICA } \\
\text { As previous. }\end{array}$ & $\begin{array}{l}\text { RIC IV/ } \\
\text { III, 34A. }\end{array}$ & $251-253$ & 6144 \\
\hline 246 & $\begin{array}{l}\text { ANT. } \\
3.16 \\
21.9 \\
11\end{array}$ & $\begin{array}{l}\text { IMP CAE C VIB TREB } \\
\text { GALLVS AVG } \\
\text { As previous. }\end{array}$ & $\begin{array}{l}\text { LIBERTAS AVGG } \\
\text { Liberitas stg.l., hld. pileus and scepter. }\end{array}$ & $\begin{array}{l}\text { RIC IV/ } \\
\text { III, } 37 .\end{array}$ & $251-253$ & 6132 \\
\hline 247 & $\begin{array}{l}\text { ANT. } \\
3.31 \\
21.1 \\
12\end{array}$ & $\begin{array}{l}\text { IMP CAE C VIB TREB } \\
\text { GALLVS AVG } \\
\text { As previous. }\end{array}$ & $\begin{array}{l}\text { LIBERTAS AVGG } \\
\text { Liberitas stg.l., hld. pileus and scepter, with } \\
\text { legs crossed, resting on column. }\end{array}$ & $\begin{array}{l}\text { RIC IV/ } \\
\text { III, } 39 .\end{array}$ & $251-253$ & 6133 \\
\hline 248 & $\begin{array}{l}\text { ANT. } \\
3.29 \\
22.9 \\
6\end{array}$ & $\begin{array}{l}\text { IMP CAE C VIB TREB } \\
\text { GALLVS AVG } \\
\text { As previous. }\end{array}$ & $\begin{array}{l}\text { PIETAS AVGG } \\
\text { Pietas, veiled, stg. 1., raising both hands; in } \\
\text { field r., star. }\end{array}$ & $\begin{array}{l}\text { RIC IV/ } \\
\text { III, } 42 .\end{array}$ & $251-253$ & 6143 \\
\hline 249 & $\begin{array}{l}\text { ANT. } \\
3.51 \\
21.4 \\
6\end{array}$ & $\begin{array}{l}\text { IMP CAE C VIB TREB } \\
\text { GALLVS AVG } \\
\text { As previous. }\end{array}$ & $\begin{array}{l}\text { PIETAS AVGG } \\
\text { As previous. }\end{array}$ & $\begin{array}{l}\text { RIC IV/ } \\
\text { III, } 42 .\end{array}$ & $251-253$ & 6142 \\
\hline 250 & $\begin{array}{l}\text { ANT. } \\
2.11 \\
19.1 \\
6\end{array}$ & $\begin{array}{l}\text { [IMP CAE C VIB TREB } \\
\text { GALLVS AVG] } \\
\text { As previous. }\end{array}$ & $\begin{array}{l}\text { [PROVI]DENTIA AVG } \\
\text { Providentia stg. 1., hld. globe and transverse } \\
\text { scepter. }\end{array}$ & $\begin{array}{l}\text { RIC IV/ } \\
\text { III, } 43 .\end{array}$ & $251-253$ & 6166 \\
\hline 251 & $\begin{array}{l}\text { ANT. } \\
3.79 \\
21.5 \\
6\end{array}$ & $\begin{array}{l}\text { IMP CAE C VIB TREB } \\
\text { GALLVS AVG } \\
\text { As previous. }\end{array}$ & $\begin{array}{l}\text { VICTORIA AVGG } \\
\text { Victory stg. 1., hld. wreath and palm. }\end{array}$ & $\begin{array}{l}\text { RIC IV/ } \\
\text { III, 48a. }\end{array}$ & $251-253$ & 6156 \\
\hline 252 & $\begin{array}{l}\text { ANT. } \\
3.54 \\
23.6 \\
7\end{array}$ & $\begin{array}{l}\text { IMP CAE C VIB TREB } \\
\text { GALLVS AVG } \\
\text { As previous. }\end{array}$ & $\begin{array}{l}\text { VICTORIA AVGG } \\
\text { As previous. }\end{array}$ & $\begin{array}{l}\text { RIC IV/ } \\
\text { III, 48a. }\end{array}$ & $251-253$ & 6157 \\
\hline 253 & $\begin{array}{l}\text { ANT. } \\
2.98 \\
22.4 \\
6\end{array}$ & $\begin{array}{l}\text { IMP CAE C VIB TREB } \\
\text { GALLVS AVG } \\
\text { As previous. }\end{array}$ & $\begin{array}{l}\text { VICTORIA AVGG } \\
\text { As previous. }\end{array}$ & $\begin{array}{l}\text { RIC IV/ } \\
\text { III, 48a. }\end{array}$ & $251-253$ & 6155 \\
\hline 254 & $\begin{array}{l}\text { ANT. } \\
3.76 \\
22.3 \\
5\end{array}$ & $\begin{array}{l}\text { IMP CAE C VIB TREB } \\
\text { GALLVS AVG }\end{array}$ & $\begin{array}{l}\text { LIBERITAS PVBLICA } \\
\text { Libertas stg. 1., hld. pileus and } \\
\text { transverse scepter. }\end{array}$ & $\begin{array}{l}\text { RIC IV/ } \\
\text { III, } 50 .\end{array}$ & $251-253$ & 6131 \\
\hline 255 & $\begin{array}{l}\text { ANT. } \\
3,51 \\
22.0 \\
6\end{array}$ & $\begin{array}{l}\text { IMP C C VIB TREB } \\
\text { GALLVS P F AVG } \\
\text { Bust, radiate, dr., } \\
\text { cuirassed, r. }\end{array}$ & $\begin{array}{l}\text { FELICI-TAS PVBLICA } \\
\text { Felicitas stg. 1., hld. caduceus and } \\
\text { cornucopiae. }\end{array}$ & $\begin{array}{l}\text { RIC IV/ } \\
\text { III, } 82 .\end{array}$ & $251-253$ & 6134 \\
\hline
\end{tabular}




\begin{tabular}{|c|c|c|}
\hline 256 & $\begin{array}{l}\text { ANT. } \\
4,10 \\
21.4 \\
12\end{array}$ & $\begin{array}{l}\text { IMP C C VIB TREB } \\
\text { GALLVS P F AVG } \\
\text { As previous; one dot } \\
\text { beneath the bust. }\end{array}$ \\
\hline 257 & $\begin{array}{l}\text { ANT. } \\
3,34 \\
22.0 \\
11\end{array}$ & $\begin{array}{l}\text { IMP C C VIB TREB } \\
\text { GALLVS AVG } \\
\text { As previous, three dots } \\
\text { beneath the bust. }\end{array}$ \\
\hline 258 & $\begin{array}{l}\text { ANT. } \\
3,83 \\
20.5 \\
5\end{array}$ & $\begin{array}{l}\text { [?]IMP C C VIB TREB } \\
\text { GALLVS PF AVG } \\
\text { As previous. }\end{array}$ \\
\hline 259 & $\begin{array}{l}\text { ANT. } \\
3,71 \\
22.3 \\
11\end{array}$ & $\begin{array}{l}\text { IMP C C VIB TREB } \\
\text { GALLVS PF AVG } \\
\text { As previous, three dots } \\
\text { beneath the bust. }\end{array}$ \\
\hline 260 & $\begin{array}{l}\text { ANT. } \\
3,55 \\
24.6 \\
11\end{array}$ & $\begin{array}{l}\text { IMP C C VIB TREB } \\
\text { GALLVS P F AVG } \\
\text { As previous, three dots } \\
\text { beneath the bust. }\end{array}$ \\
\hline
\end{tabular}

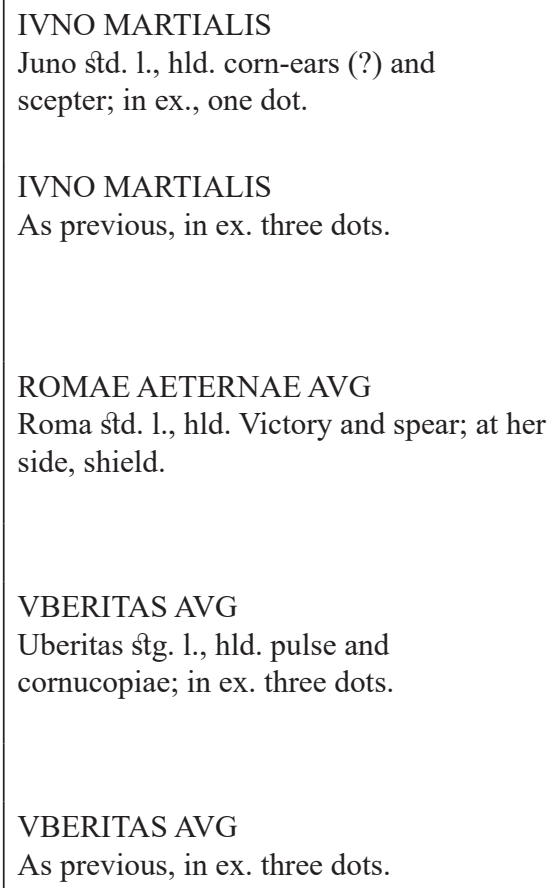

\begin{tabular}{|c|c|c|}
\hline 261 & $\begin{array}{l}\text { ANT. } \\
3,25 \\
23.8 \\
6\end{array}$ & $\begin{array}{l}\text { IMP C C VIB TREB } \\
\text { GALLVS AVG } \\
\text { As previous. }\end{array}$ \\
\hline 262 & $\begin{array}{l}\text { ANT. } \\
3,13 \\
24.1 \\
11\end{array}$ & $\begin{array}{l}\text { IMP C C VIB TREB } \\
\text { GALLVS AVG } \\
\text { As previous. }\end{array}$ \\
\hline 263 & $\begin{array}{l}\text { ANT. } \\
3,96 \\
21.7 \\
8\end{array}$ & $\begin{array}{l}\text { IMP C C VIB TREB } \\
\text { GALLVS AVG } \\
\text { As previous. }\end{array}$ \\
\hline 264 & $\begin{array}{l}\text { ANT. } \\
3,19 \\
24.3 \\
1\end{array}$ & $\begin{array}{l}\text { IMP C C VIB TREB } \\
\text { GALLVS AVG } \\
\text { As previous. }\end{array}$ \\
\hline 265 & $\begin{array}{l}\text { ANT. } \\
3,24 \\
22.4 \\
7\end{array}$ & $\begin{array}{l}\text { IMP C C VIB TREB } \\
\text { GALLVS AVG } \\
\text { As previous. }\end{array}$ \\
\hline 266 & $\begin{array}{l}\text { ANT. } \\
3,47 \\
21.5 \\
11\end{array}$ & $\begin{array}{l}\text { IMP C C VIB TREB } \\
\text { GALLVS AVG } \\
\text { As previous. }\end{array}$ \\
\hline
\end{tabular}

IVNO MARTIALIS

Juno std. 1., hld. corn-ears (?) and sceptre.

IVNO MARTIALIS

As previous.

\section{LIBERITAS PVBLICA}

Liberitas stg.l., hld. pileus and

transverse scepter

\section{PAX AETERNA}

Pax stg. 1., hld. branch and transverse scepter.

\section{PAX AETERNA}

As previous.

PIETAS AVGG

Pietas, veiled, stg. 1., raising both hands; to left altar.
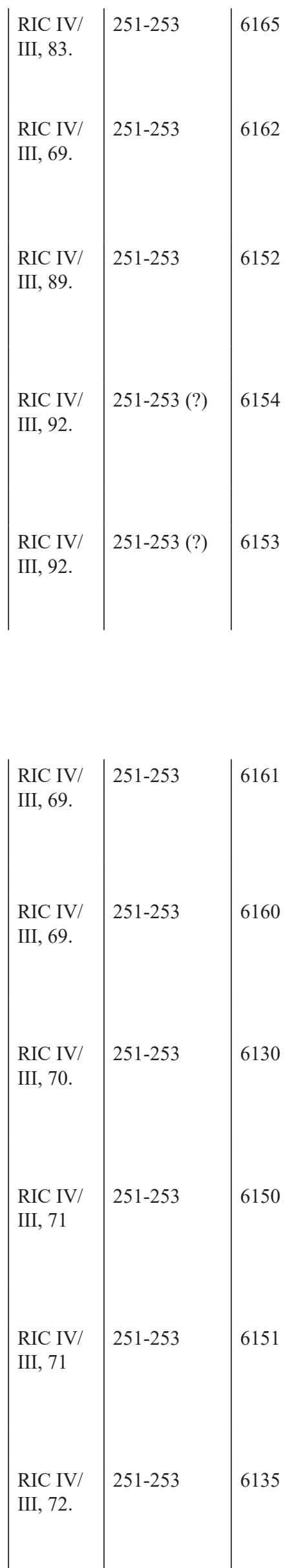


\begin{tabular}{|l|l|l}
267 & ANT. & IMP C C VIB TREB \\
& 3,26 & GALLVS AVG \\
& 22.6 & As previous.. \\
& 7 & \\
& & \\
& & \\
& ANT. & IMP C C VIB TREB \\
& 3,59 & GALLVS AVG \\
& 1 & As previous. \\
& & \\
& ANT. & IMP C C VIB TREB \\
& 3,51 & GALLVS AVG \\
& 22.8 & As previous. \\
& 6 &
\end{tabular}
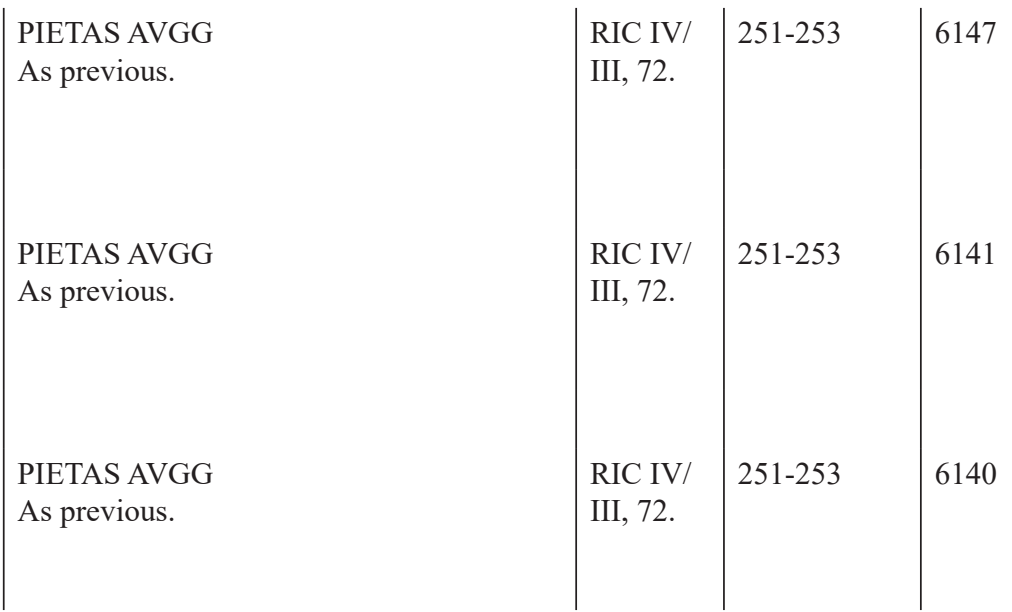

\section{VOLUSIAN}

Cat. 270-291)

\section{Mint of Rome}

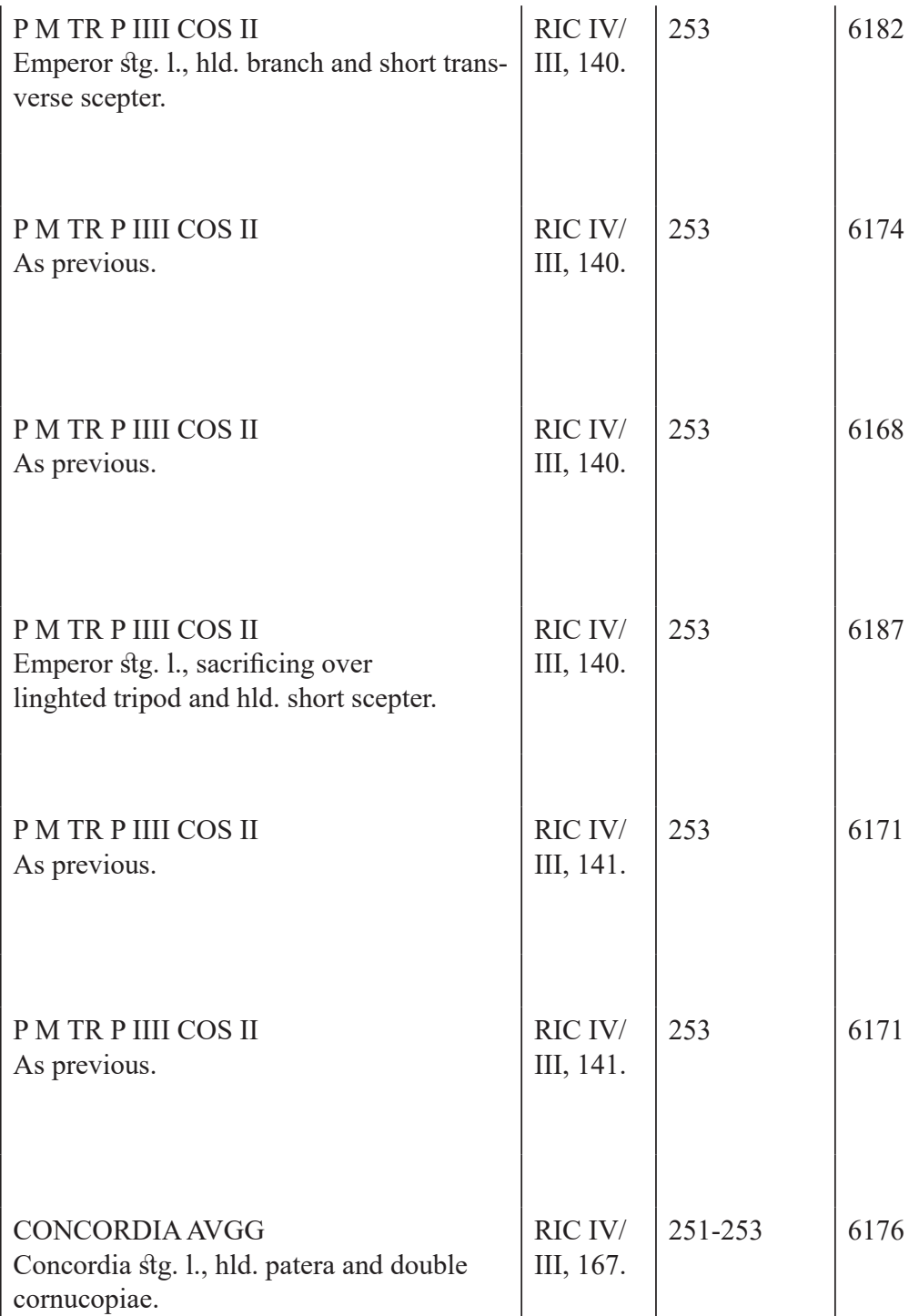

253

6182

RIC IV/

III, 140.

253

6174

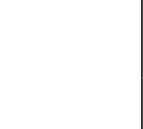

RIC IV/

III, 140.

253

6168

RIC IV/

253

6187

III, 140.

253

6171

III, 141.

III, 141.

253

6171

RIC IV/

251-253

III, 167.

6176 


\begin{tabular}{|c|c|c|c|c|c|c|}
\hline 277 & $\begin{array}{l}\text { ANT. } \\
2,79 \\
20.5 \\
1\end{array}$ & $\begin{array}{l}\text { IMP CAE C VIB } \\
\text { VOLVSIANO AVG } \\
\text { As previous. }\end{array}$ & $\begin{array}{l}\text { CONCORDIA AVGG } \\
\text { As previous. }\end{array}$ & $\begin{array}{l}\text { RIC IV/ } \\
\text { III, } 167 .\end{array}$ & 251-253 & 6176 \\
\hline 278 & $\begin{array}{l}\text { ANT. } \\
3,18 \\
21.5 \\
7\end{array}$ & $\begin{array}{l}\text { IMP CAE C VIB } \\
\text { VOLVSIANO AVG } \\
\text { As previous. }\end{array}$ & $\begin{array}{l}\text { CONCORDIA AVGG } \\
\text { As previous. }\end{array}$ & $\begin{array}{l}\text { RIC IV/ } \\
\text { III, } 167 .\end{array}$ & $251-253$ & 6167 \\
\hline 279 & $\begin{array}{l}\text { ANT. } \\
2,76 \\
20.3 \\
6\end{array}$ & $\begin{array}{l}\text { IMP CAE C VIB } \\
\text { VOLVSIANO AVG } \\
\text { As previous. }\end{array}$ & $\begin{array}{l}\text { CONCORDIA AVGG } \\
\text { Concordia std. 1., hld. patera and double } \\
\text { cornucopiae. }\end{array}$ & $\begin{array}{l}\text { RIC IV/ } \\
\text { III, } 168 .\end{array}$ & $251-253$ & 6173 \\
\hline 280 & $\begin{array}{l}\text { ANT. } \\
3,19 \\
21.3 \\
1\end{array}$ & $\begin{array}{l}\text { IMP CAE C VIB } \\
\text { VOLVSIANO AVG } \\
\text { As previous. }\end{array}$ & $\begin{array}{l}\text { CONCORDIA AVGG } \\
\text { As previous. }\end{array}$ & $\begin{array}{l}\text { RIC IV/ } \\
\text { III, } 168 .\end{array}$ & $251-253$ & 6178 \\
\hline 281 & $\begin{array}{l}\text { ANT. } \\
2,70 \\
20.4 \\
1\end{array}$ & $\begin{array}{l}\text { IMP CAE C VIB } \\
\text { VOLVSIANO AVG } \\
\text { As previous. }\end{array}$ & $\begin{array}{l}\text { CONCORDIA AVGG } \\
\text { As previous. }\end{array}$ & $\begin{array}{l}\text { RIC IV/ } \\
\text { III, } 168 .\end{array}$ & $251-253$ & 6183 \\
\hline 282 & $\begin{array}{l}\text { ANT. } \\
3,32 \\
21.2 \\
12\end{array}$ & $\begin{array}{l}\text { IMP CAE C VIB } \\
\text { VOLVSIANO AVG } \\
\text { As previous. }\end{array}$ & $\begin{array}{l}\text { CONCORDIA AVGG } \\
\text { As previous, but star r. field. }\end{array}$ & $\begin{array}{l}\text { RIC IV/ } \\
\text { III, } 169 .\end{array}$ & $251-253$ & 6169 \\
\hline 283 & $\begin{array}{l}\text { ANT. } \\
2,15 \\
22.4 \\
6\end{array}$ & $\begin{array}{l}\text { IMP CAE C VIB } \\
\text { VOLVSIANO AVG } \\
\text { As previos. }\end{array}$ & $\begin{array}{l}\text { PAX AVGG } \\
\text { Pax stg. 1., hld. branch and transverse } \\
\text { scepter. }\end{array}$ & $\begin{array}{l}\text { RIC IV/ } \\
\text { III, } 179 .\end{array}$ & $251-253$ & 6180 \\
\hline 284 & $\begin{array}{l}\text { ANT. } \\
4,28 \\
22.4 \\
7\end{array}$ & $\begin{array}{l}\text { IMP CAE C VIB } \\
\text { VOLVSIANO AVG } \\
\text { As previous. }\end{array}$ & $\begin{array}{l}\text { PAX AVGG } \\
\text { As previous. }\end{array}$ & $\begin{array}{l}\text { RIC IV/ } \\
\text { III, } 179 .\end{array}$ & $251-253$ & 6177 \\
\hline 285 & $\begin{array}{l}\text { ANT. } \\
2,93 \\
22.1 \\
12\end{array}$ & $\begin{array}{l}\text { IMP CAE C VIB } \\
\text { VOLVSIANO AVG } \\
\text { As previous. }\end{array}$ & $\begin{array}{l}\text { PAX AVGG } \\
\text { As previous, but star in filed. }\end{array}$ & $\begin{array}{l}\text { RIC IV/ } \\
\text { III, } 180 .\end{array}$ & $251-253$ & 6170 \\
\hline 286 & $\begin{array}{l}\text { ANT. } \\
2,49 \\
21.0 \\
1\end{array}$ & $\begin{array}{l}\text { IMP CAE C VIB } \\
\text { VO[LVSIANO AVG] } \\
\text { As previous. }\end{array}$ & $\begin{array}{l}\text { PIETAS AVGG } \\
\text { Pietas veiled, stg.1., raising both hands; to } \\
\text { 1., altar. }\end{array}$ & $\begin{array}{l}\text { RIC IV/ } \\
\text { III, } 182\end{array}$ & $251-253$ & 6172 \\
\hline 287 & $\begin{array}{l}\text { ANT. } \\
1,96 \\
19.0 \\
7\end{array}$ & $\begin{array}{l}\text { IMP CAE C VIB } \\
\text { VO[LVSIANO AVG] } \\
\text { As previos. }\end{array}$ & $\begin{array}{l}\text { SALVS A[ ] } \\
\text { Salus stg. r., feeding out of patera snake } \\
\text { held in arms. }\end{array}$ & $\begin{array}{l}\text { RIC IV/ } \\
\text { III, } 184 .\end{array}$ & $251-253$ & 6179 \\
\hline
\end{tabular}




\begin{tabular}{|l|l|l}
288 & ANT. & IMP CAE C VIB \\
& 3,95 & VOLVSIANO AVG \\
& 24.3 & As previous. \\
& 6 & \\
& & \\
& & \\
& ANT. & IMP CAE C VIB \\
& 4,56 & VOLVSIANO AVG \\
& 21.3 & As previos. \\
& 12 &
\end{tabular}

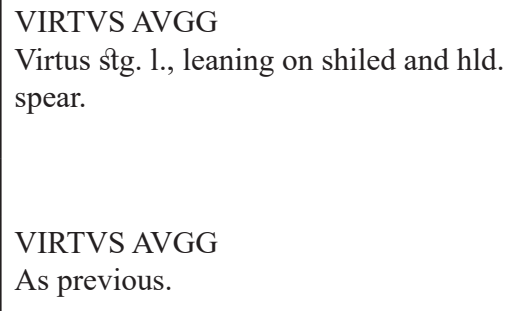

(Cat. 290)

\section{Mint of Milan}

\begin{tabular}{|l|l|l}
290 & ANT. & IMP C C VIB \\
& 4,26 & VOLVSIANO AVG \\
& 21.5 & As previous. \\
& 11 &
\end{tabular}

FELICITAS PVBL

Felicitas stg. 1., hld. long caduceus and cornucopiae. \begin{tabular}{|l|l|l|} 
RIC IV/ & $251-253$ & 6184 \\
III, 205. & &
\end{tabular}

(Cat. 291)

\section{Mint of Antioch}

ADVENTVS AVG

Volusian on horseback 1., raising $\mathrm{r}$.

RIC IV/

$251-253$

6186

hand and hld. spear.

\section{AEMILIAN}

(cat. 292-297)

\section{Mint of Rome}

\begin{tabular}{|l|l|l}
292 & ANT. & IMP AEMILIANVS \\
& 2,69 & PIVS FEL AVG \\
& 21.9 & $\begin{array}{l}\text { Bust, radiate, dr. } \\
\text { cuirassed, r. }\end{array}$ \\
& 1 & \\
& ANT. & IMP AEMILIANVS \\
& 2,56 & PIVS FEL AVG \\
& 1.3 & As previous. \\
& 1 & \\
& ANT. & IMP AEMILIANVS \\
& 3,29 & PIVS FEL AVG \\
& 22.6 & As previous. \\
& 1 & \\
& & \\
& ANT. & IMP AEMILIANVS \\
& 3.17 & PIVS FEL AVG \\
& 22.0 & As previous. \\
& 6 & \\
& & \\
& & \\
& ANT. & \\
& 3.35 & IMP AEMILIANVS \\
& 21.0 & PIVS FEL AVG \\
& &
\end{tabular}

III, 224.

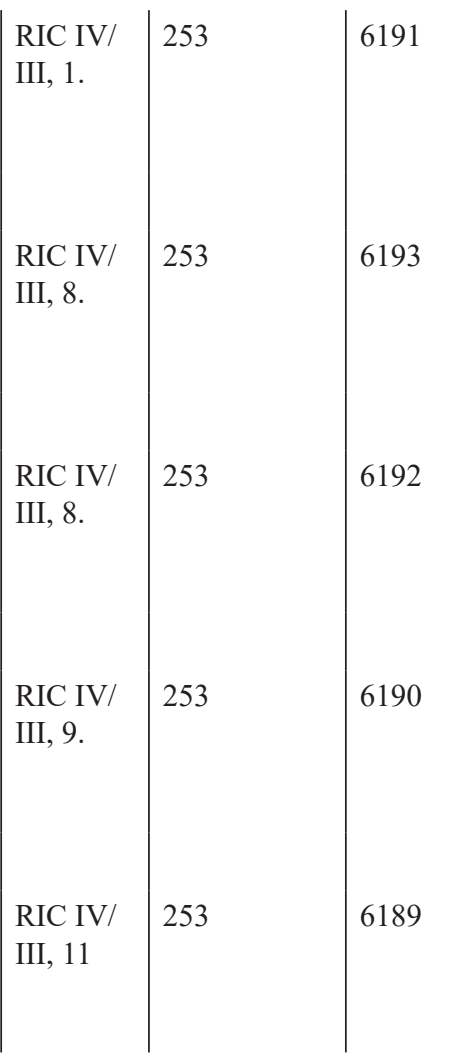


\begin{tabular}{|l|l|l}
297 & ANT. & IMP AEMILIANVS \\
& 4.17 & PIVS FEL AVG \\
& 21.7 & As previous. \\
7 &
\end{tabular}

\begin{tabular}{|l|l|l}
298 & ANT. & IMP C P LIC \\
& 2.72 & VALERIANVS AVG \\
& 23.1 & Bust, radiate, dr., r. \\
& 6 & \\
& & \\
& ANT. & IMP C P LIC \\
& 3.26 & VALERIANVS AVG \\
& 20.6 & As previous. \\
& 5 & \\
& & \\
& ANT. & IMP C P LIC \\
& 3.47 & VALERIANVS AVG \\
& 26.3 & As previous. \\
& 7 &
\end{tabular}

VIRTVS AVG

Virtus stg.1., r. foot on helmet, hld.

branch and spear.

\section{VALERIAN}

(cat. 298-300)

\section{Mint of Rome}

\section{SALVS AVGG}

Salus stg. 1., hld. scepter in 1. hand, feeding serpent rising from altar.

VICTORIA AVG

Victory stg. 1., hld. wreath and palm.

VICTORIA AVG

As previous.
RIC IV/

III, 12.

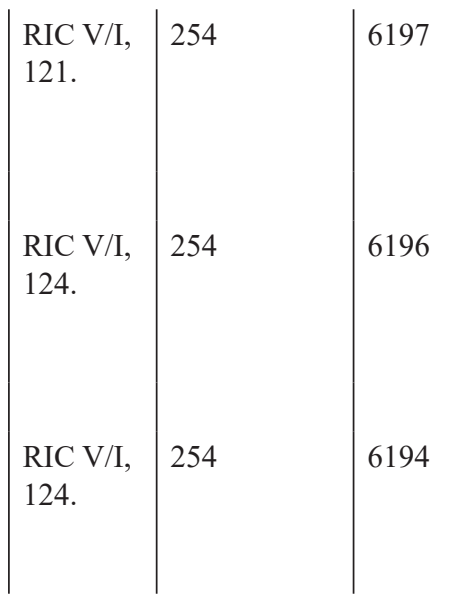

(cat. 301-304)

\section{Mint of Milan/Mediolanum}

\begin{tabular}{|l|l|l}
301 & ANT. & IMP VALERIANVS P \\
& 3.29 & AVG \\
& 23.6 & As previous. \\
& 7 & \\
& & \\
& ANT. & IMP P LIC \\
& 2.03 & VALERIANIANO AVG \\
& 7 & As previous. \\
& & \\
& ANT. & IMP P LIC \\
& 2.85 & VALERIANIANO AVG \\
& 23.2 & As previous. \\
& 7 & \\
& & \\
304 & ANT. & IMP VALERIANVS P \\
& 3.83 & AVG \\
& 21.8 & As previous. \\
& 11 &
\end{tabular}

FIDES MILITVM
As previous.

FIDES MILITVM
As previous.

CONCOR LEGG
Concordia std. 1., head r., hld.

patera and cornucopiae.

FIDES MILITVM

Fides stg. 1., hld. standard and

transverse ensign.

FIDES MILITVM

As previous.

MARINIANA

(Striking under Valerian)

(cat. 305)

\section{Mint of Rome}

\begin{tabular}{|l|l|l}
305 & ANT. & DIVAE MARINIANAE \\
& 3.11 & Veiled bust r., on cresent. \\
& 71.7 &
\end{tabular}
CONSECRATIO

Peacock flying $r$ carrying empress to heaven.

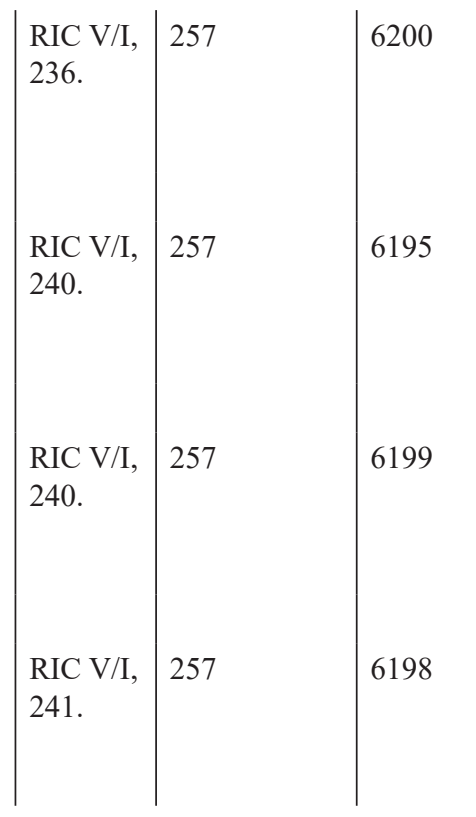

6201 
GALLIENUS (join reign)

(cat. 306)

Mint of Milan/Mediolanum

\begin{tabular}{|l|l|l|l|l|l|l|}
306 & ANT. & IMP GALLIENVS P & SALVS AVGG & RIC V/I, 397. & $257-258$ & 6202 \\
& 2.82 & AVG & Salus stg.l., feeding serpent & VASIĆ 2005: & $(254-$ Vi- & 48, cat. 1585- \\
& 20.6 & Radiate, cuir. bust, r. & rising from altar and hld. scepter. & $1591 ;$ & \\
\hline
\end{tabular}

\section{REFERENCES}

\section{АРСЕНИЕВИТ 2004}

Арсениевић, М., Периоди похрањивања остава римског новца током III века н.е. у Горњој Мезији, Гласник Српског Археолошког Друштва 20, 225-234.

ATAHACOBA 2010

Атанасова, И., Случаен наод со кремација од Локубија, Macedoniae Acta Archaeologica 19, Skopje, 271-274.

\section{BITRAK 2016}

Bitrak, S., Монетарна циркулација на Плаошник - Охрид во римскиот империјален период : (од I до првата половина на IV век) [Monetary Circulation at Plaošnik-Ohrid in the Roman Imperial Period ( $1^{\text {st }}$ century to the first half of the $4^{\text {th }}$ century AD)] (Ohrid: Ohrid NU Zavod za zaštita na spomenicite na kulturata i Muzej).

BORIĆ BREŠKOVIĆ/STAMENKOVIĆ 2008

Borić Brešković, B./Stamenković, S., Roman Antoniniani from Village Supska near Ćuprija (Supska I), Numizmatičar 26/27, 157-207.

ВОЈВОДА/СРНДАКОВИЋ 2015

Војвода, М./Срндаковић, А. Д., Остава денара и антонинијана из села Сикирица код Параћина (Секирица III), Numizmatičar 33, 31-88.

\section{JOSIFOVSKI 2001}

Josifovski, P., Roman Mint of Stobi [Римската монетарница во Стоби] (Skopje: Narodna Banka na Republika Makedonija).

JOSIFOVSKI 2001 (a)

Josifovski, P., Activity of the Roman Mint of Stobi. In: Grozdanov C. (ed.): Coins and mints in Macedonia. Proceedings of the symposium held in honour of the 80the birthday and 50-th anniversary of the scholarly and educational work of Ksente
Bogoev, member of the Macedonian Academy of Arts and Sciences (Skopje: MANU), 53-67.

JOSIFOVSKI 2010

Josifovski, P., The Hoard Containing Denarius and Antoninianus from the Roman Forum. In: Shurbanoska, M./Jakimovski, A. 9eds.): Forum romanum Stobis II (Skopje), 82-89.

\section{KEPESKI 1993}

Кепески, К., Депо од римски монети од Стибера, Macedoniae Acta Archaeologica 13, 183-190.

КЕРАМИДЧИЕВ/АЛЕКСОВА 1966

Керамидчиев, А./Алексова, Б., Монетите низ векови (Скопје).

KERAMIDČIEV 1972

Keramidčiev, A., Považni otkritija na numizmatikata vo Makedonija vo poslednite dve i pol decenii, Arheološki Vestnik 23, 6973 .

\section{KERAMIDČIEV 1973}

Keramidčiev, A., Tri ostavi so moneti od rimsko vreme najdeni vo Makedonija, Numizmatičke vijesti 31, 25-33.

KREMYDI-SICILIANOU 2004

Kremydi-Sicilianou, S., Patterns of Monetary Circulation in Roman Macedonia. The Hoard Evidence, Eulimene 5, 135-149.

\section{KUBITSCHEK 1908}

Kubitschek, W.: Ein Denarfund aus der Gegend von Üsküb (Albanien), Numismatische Zeitschrift 41, 37-54.

KUZMANOVIČ COLL.

Josifovski, P., Macedonian Numismatic Collection. The Kuzmanović Collection: Stobi,Vol. 1, Skopje 2010.

МИКУЛЧИК 1999

Микулчиќ, И., Антички градови во Македонија (Skopje: Makedonska 
akademija na naukite i umetnostite).

MIRNIK 1981

Mirnik, I., Coin Hoards in Yugoslavia [BAR International series 95] (Oxford: BAR).

OHRIDSKA BANKA COLL. 2007

Josifovski, P./Hadži-Maneva, M./

Razmovska Bačevska, D., Coins from the Collection of Ohridska banka (Ohrid: Ohridska banka).

PAPAZOGLOU 1986

Papazoglou F., Oppidum Stobi civium Romanorum et municipium Stobensium, Chiron 16, 213-237.

PAPAZOGLOU 1988

Papazoglou F., Les villes de Macédoine à l' époque Romaine (Paris: de Boccard).

РАЗМОВСКА БАЧЕВСКА 2002

Размовска Бачевска, Д., Нумизматичките наоди од југозападниот дел на Репблика Македонија од V век п.н.е. до XIV век, Охрид.

RIC

Mattingly, H./Sydenham E.A., The Roman Imperial Coinage, IV.1, Pertinax to Geta, (London: Spink \& Son Ltd).

Mattingly, H./Sydenham E.A/Sutherland C.H.V., The Roman Imperial Coinage, IV.2, Macrinus to Pupienus (London: Spink \& Son Ltd).

Mattingly, H./Sydenham E.A/Sutherland C.H.V., The Roman Imperial Coinage, IV.3, Gordian III to Uranius Antoninus (London: Spink \& Son Ltd).

Webb, P.H., The Roman Imperial Coinage, V.1, Valerian to Florian (London: Spink \& Son Ltd).

САНЕВ/САРЖОСКИ 1981

Санев, В./Саржоски С., Ископувања на внатрешниот бедем во Стоби, 1972 - 1974. In: Aleksova, B./Wiseman, J. (eds.): Studies in the Antiquities of Stobi 3 (Priceton: University Press), 229-234.

SHURBANOSKA/JAKIMOVSKI 2007

Shurbanoska, M./Jakimovski, A., Forum romanum Stobis I, Skopje.

SHURBANOSKA/JAKIMOVSKI 2010

Shurbanoska, M./Jakimovski, A., Forum romanum Stobis II, Skopje.

TOURATSOGLOU 1993

Touratsoglou,Y., The Coin Circulation in Ancient Macedonia (ca. 200 B.C. - 268-286 A.D.). The Hoard Evidence (Athens: Hellenic Numismatic Society).

\section{TOURATSOGLOU 2006}

Touratsoglou, I., Greece and the Balkans before the end of antiquity. An approach to history and economics in an age of crisis and mutation. Prompted by the Larisa/1992 hoard (Athens: Hellenic Numismatic Society).

VASIĆ 1972

Vasić, M., Ostave 247 i 254 godine u Narodnom muzeju u Beogradu, Arheološki Vestnik 23, 57-66.

\section{VASIĆ 2005}

Vasić, M., Ostava denara i antoninijana iz Donjeg Crniljeva / A Hoard of Denarii and Antoninians from Donje Crniljevo (Beograd: Arheološki Institut). 


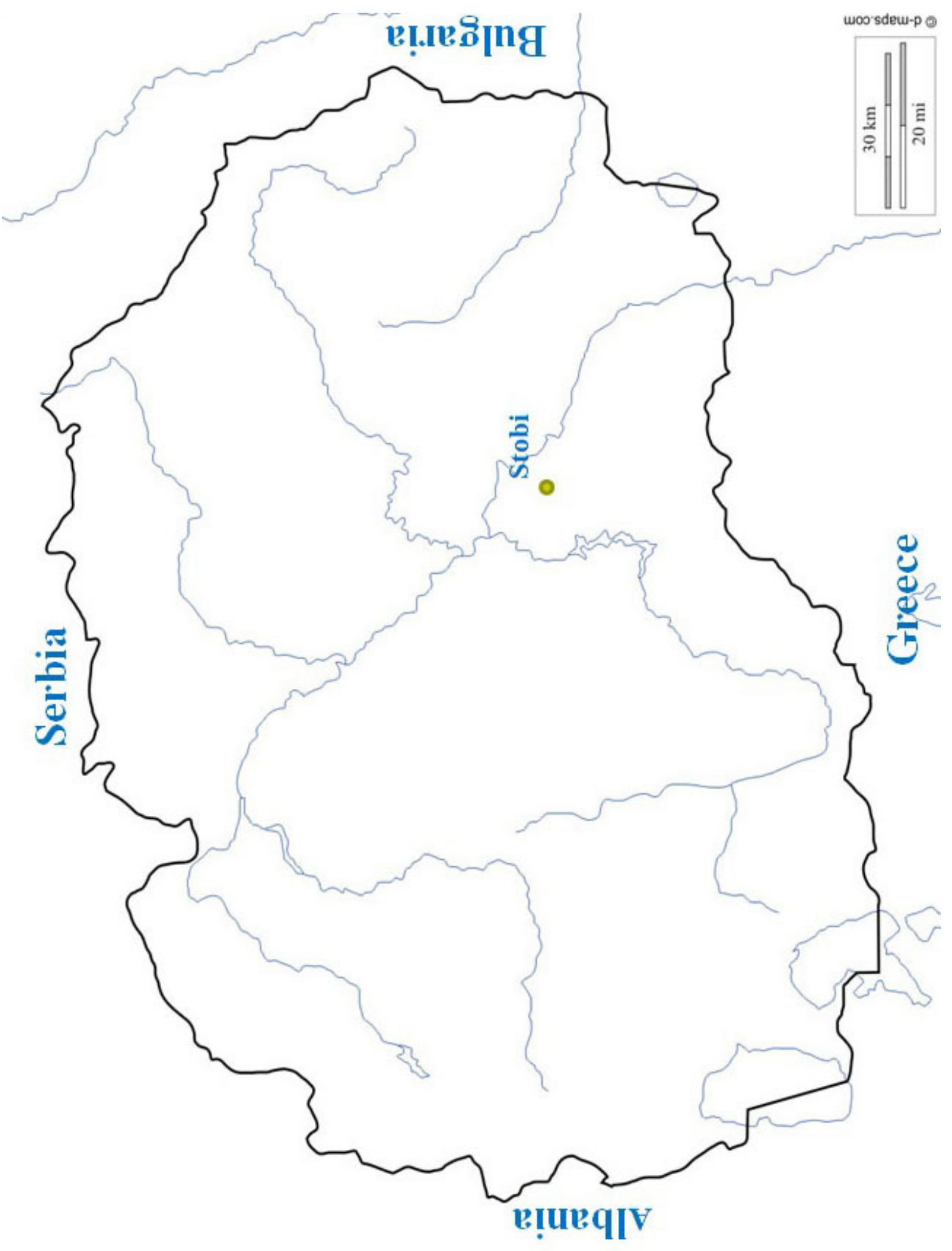



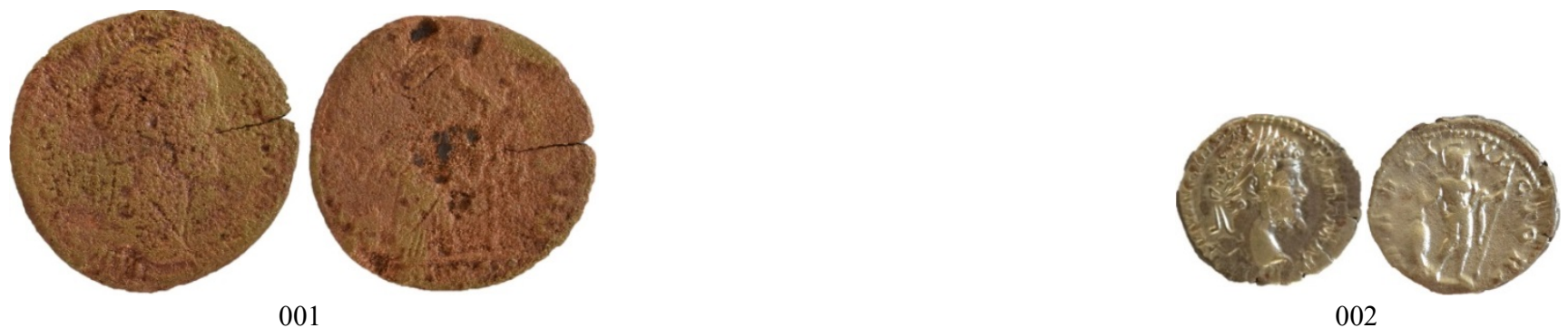

002

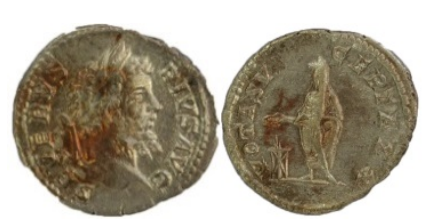

003

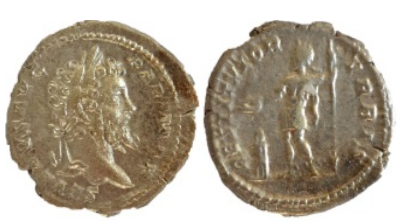

004

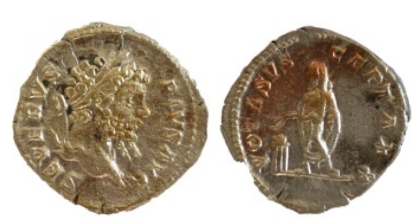

005

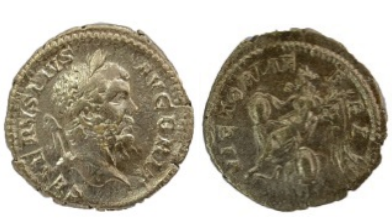

006

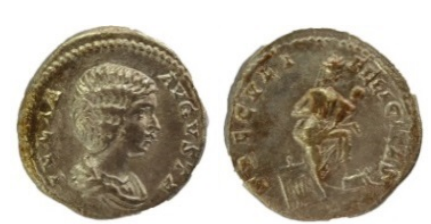

007

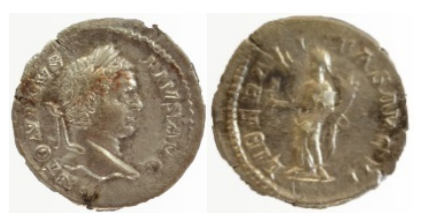

011

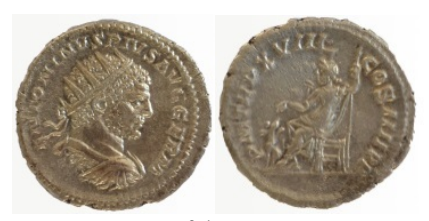

015

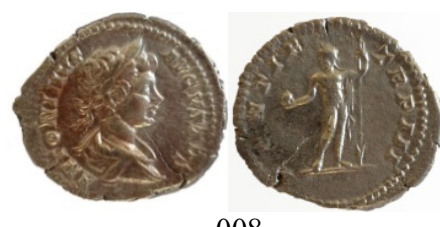

008

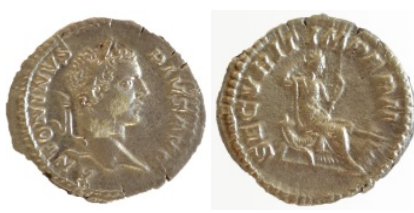

012

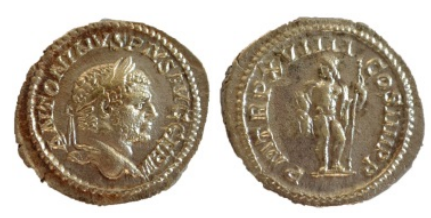

016

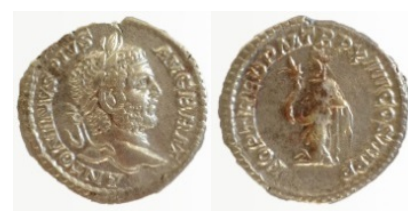

013

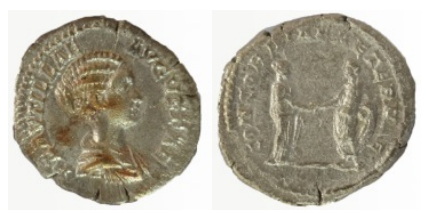

017
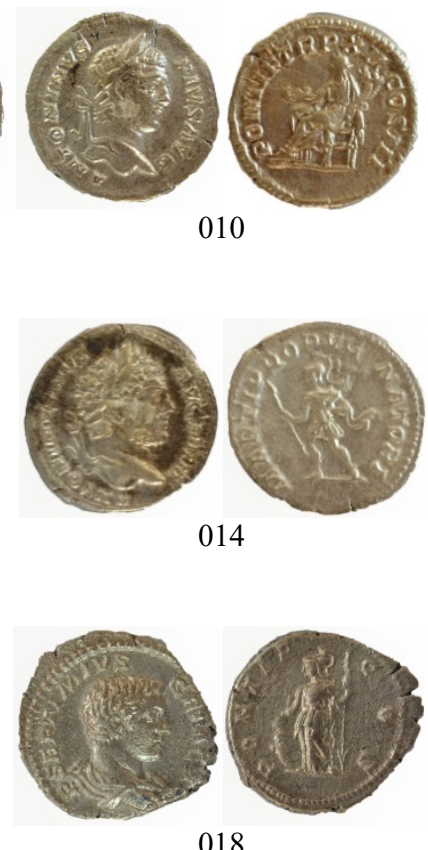

018

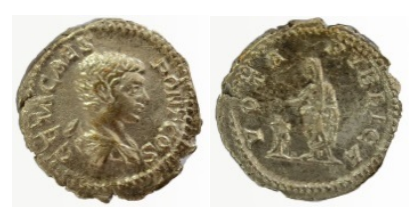

019

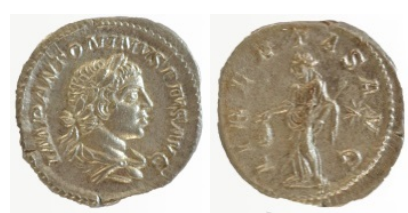

023

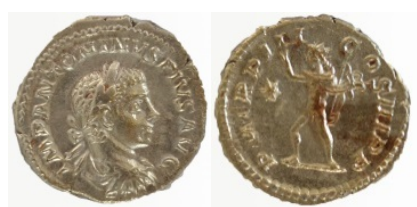

020

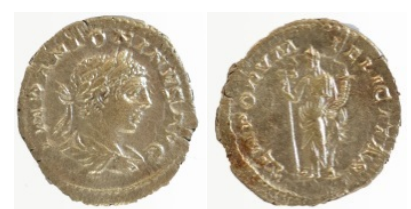

024

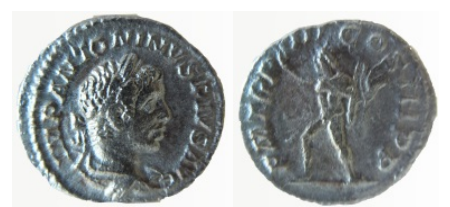

021

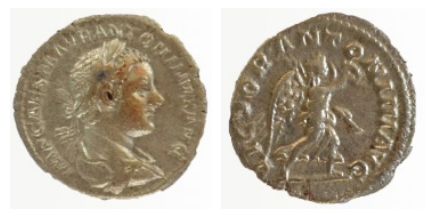

025

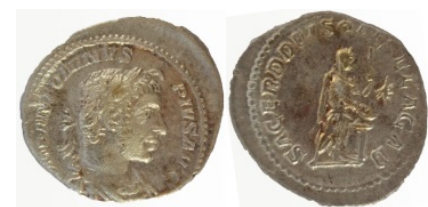

022

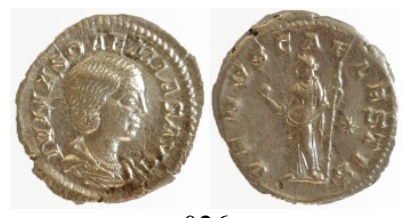




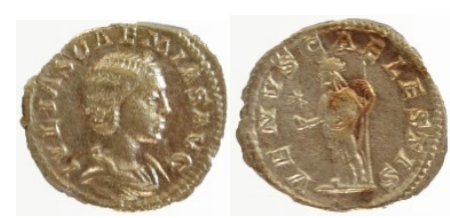

027

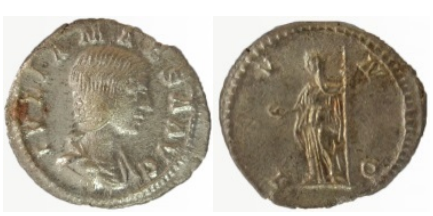

028

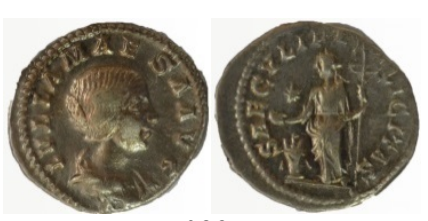

029

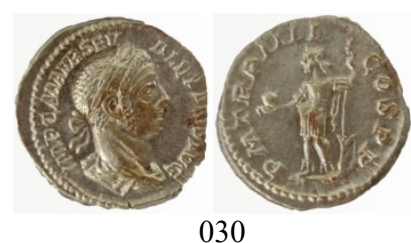

030

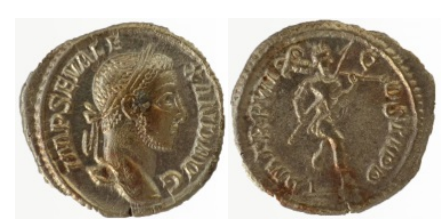

031

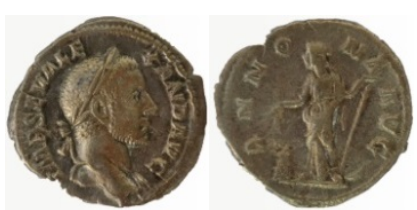

035

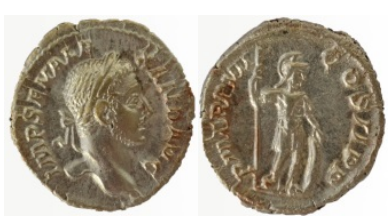

032

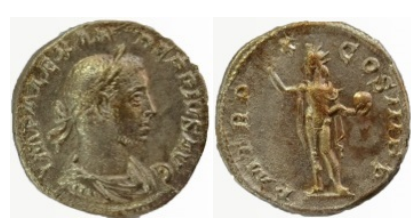

033

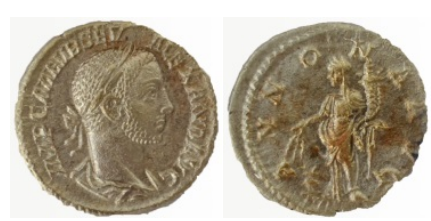

034

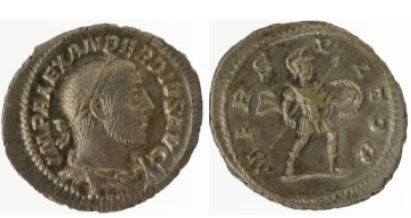

036

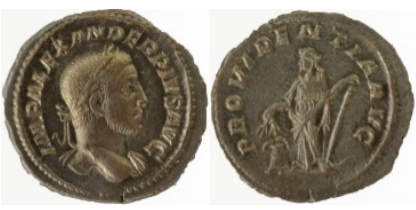

037

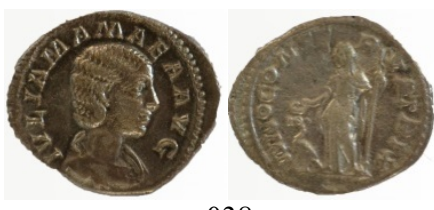

038

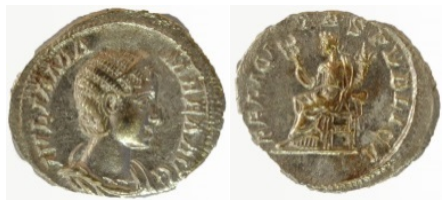

039

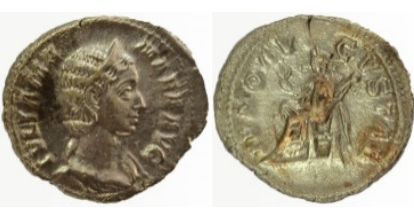

040

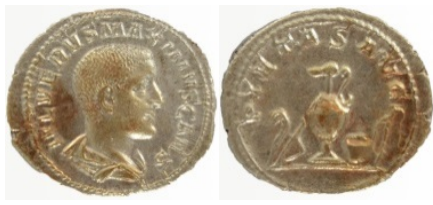

041

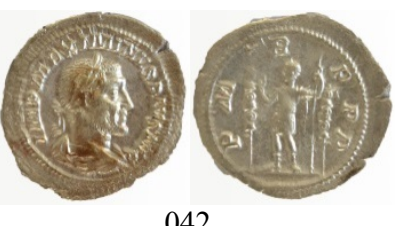

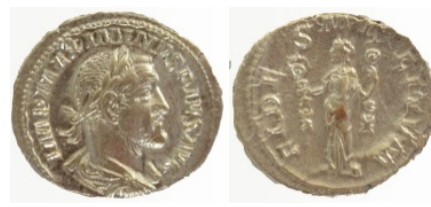

043

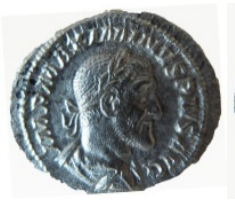

044

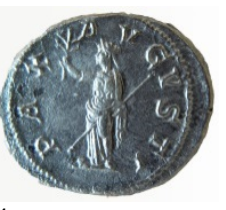

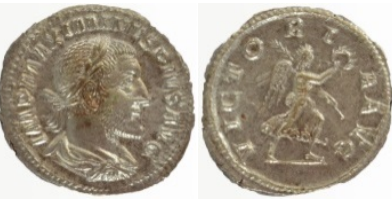

045

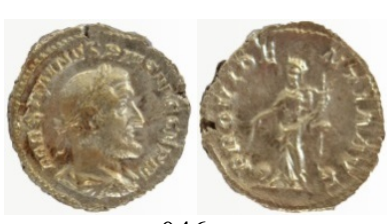

046

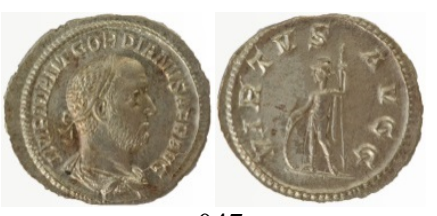

047

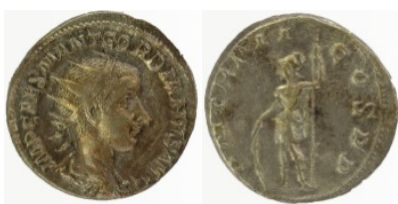

051

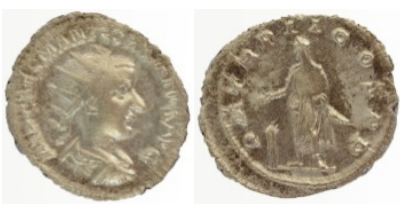

055

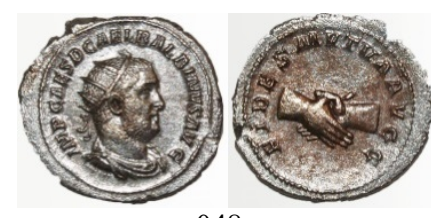

048

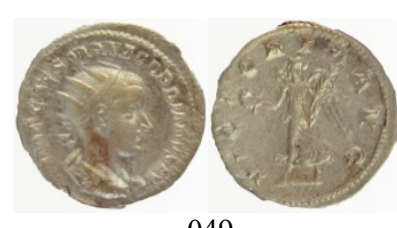

049

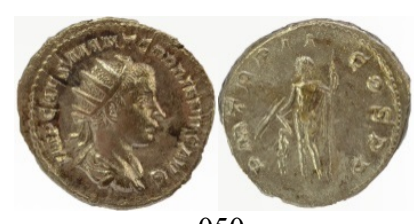

050

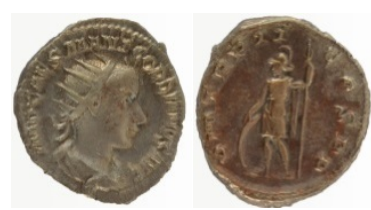

052

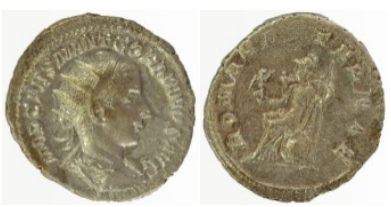

056

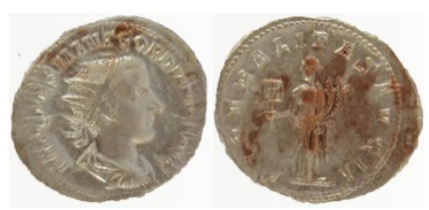

053

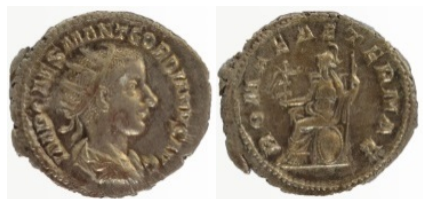

057

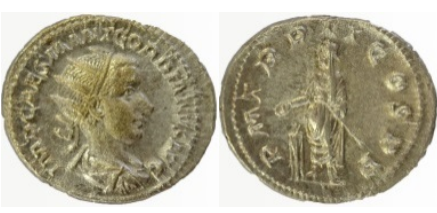

054

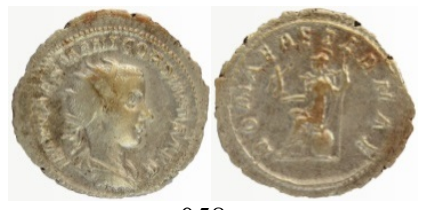




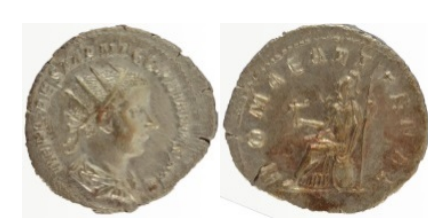

059

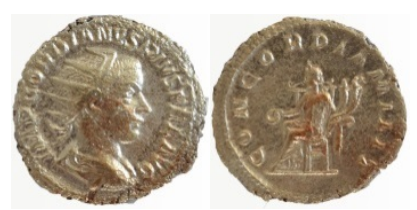

063

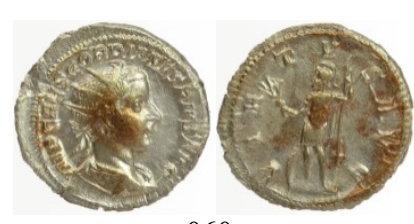

060

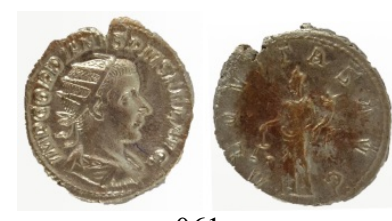

061

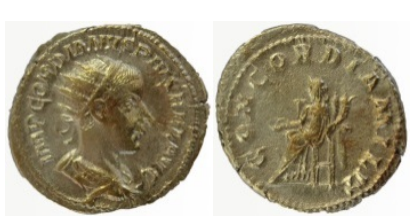

062

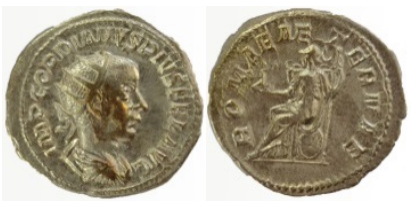

064

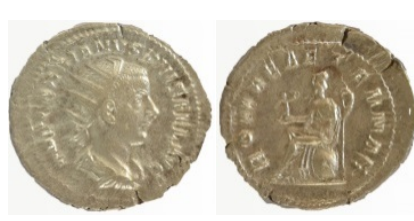

065

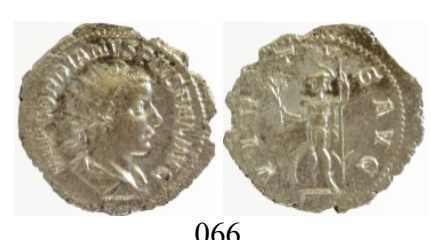

066

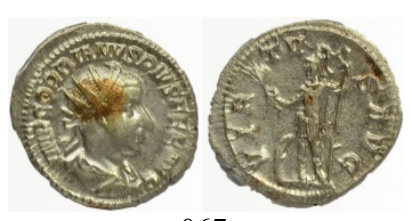

067

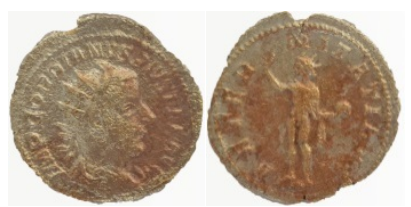

071

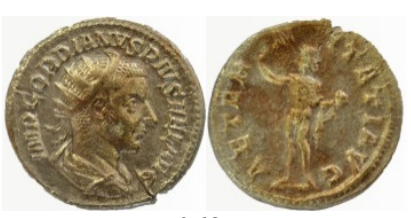

068

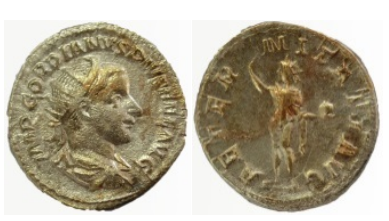

069

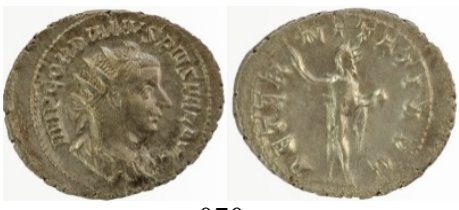

070

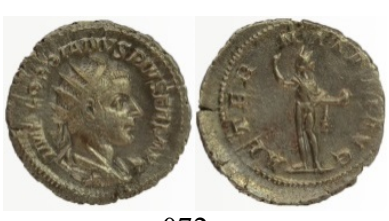

072

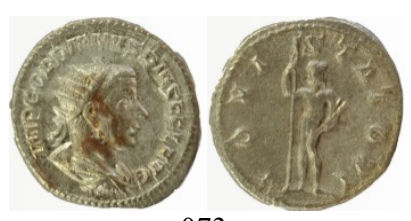

073

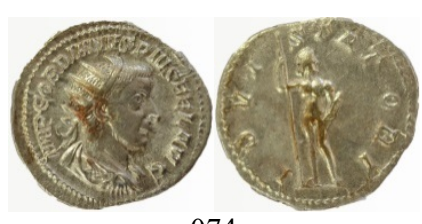

074

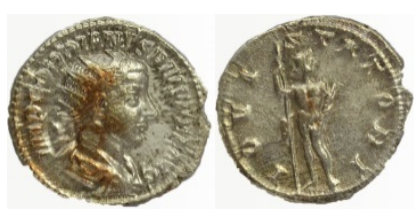

075

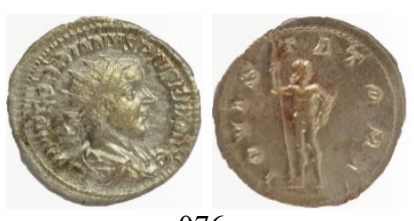

076

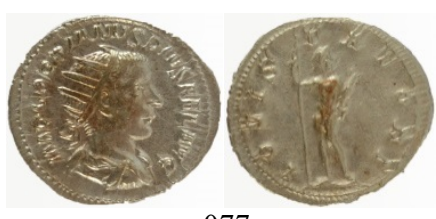

077

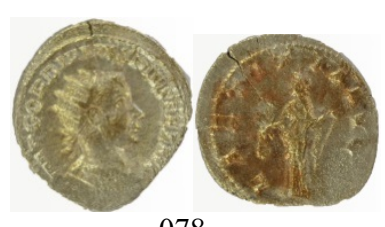

078

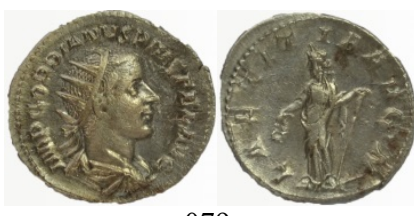

079

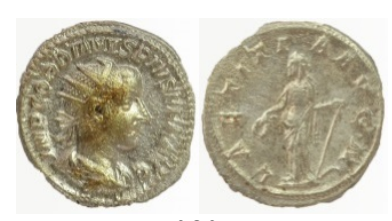

080

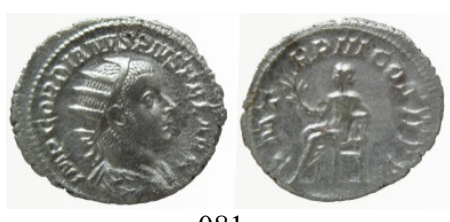

081

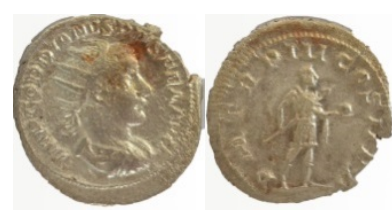

085

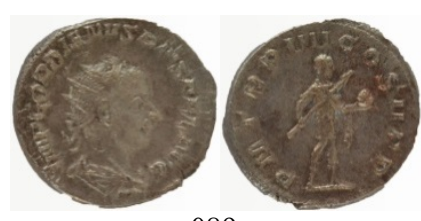

089

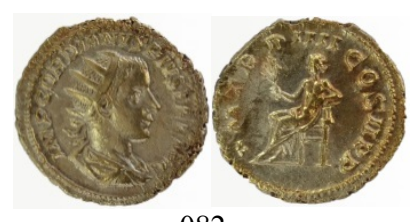

082
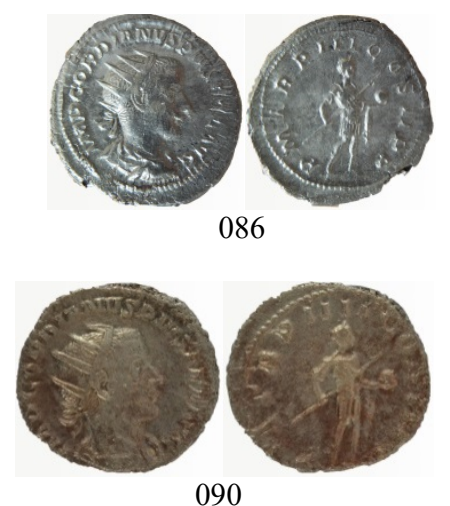


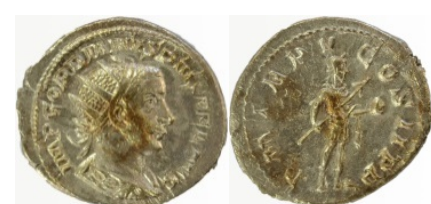

091

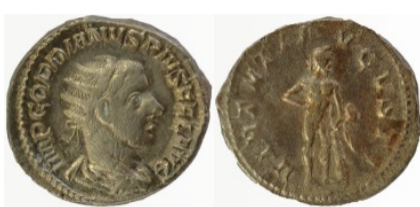

095

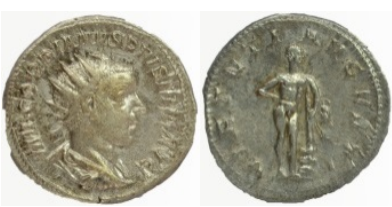

099

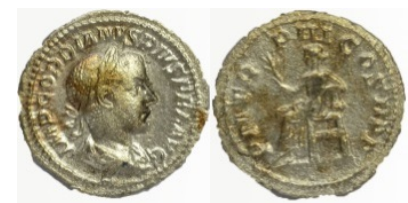

103

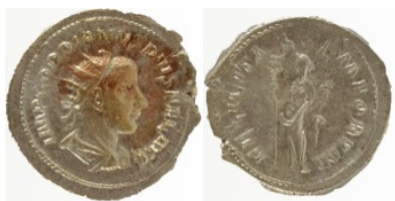

107

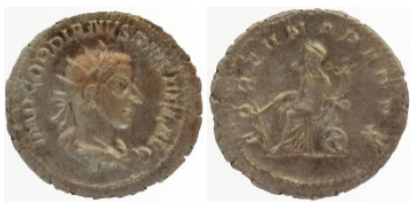

111

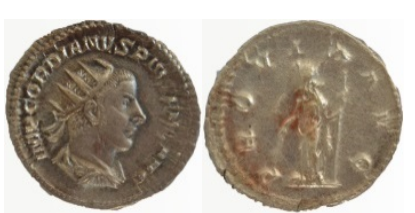

115

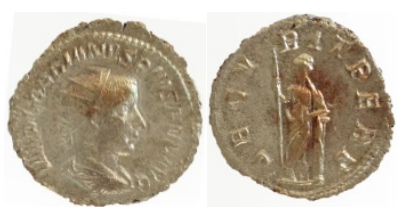

119

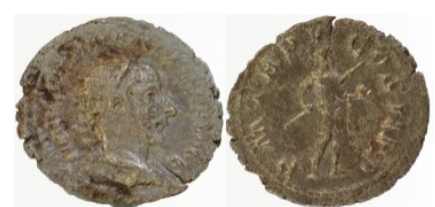

092

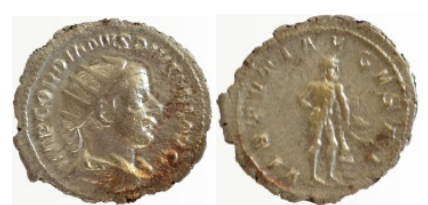

096

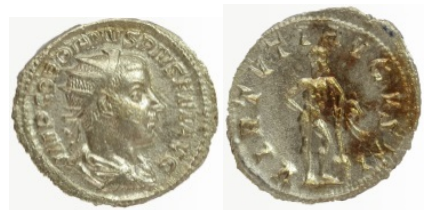

100

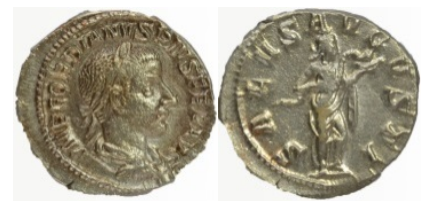

104

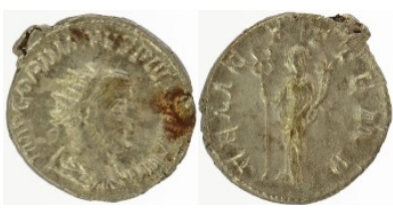

108

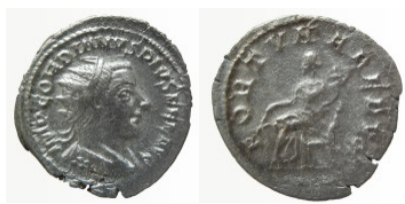

112

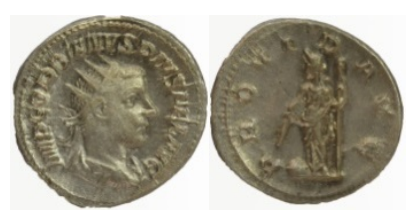

116

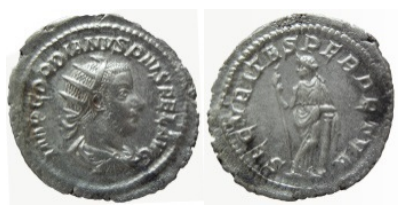

120

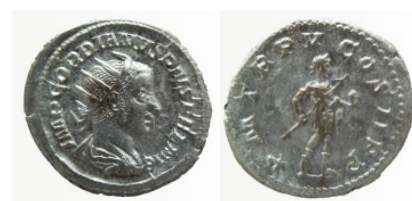

093

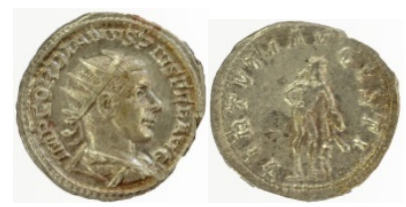

094

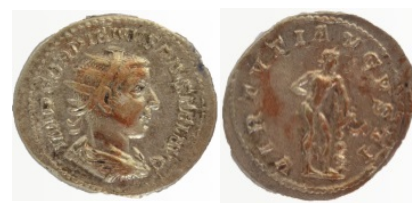

097

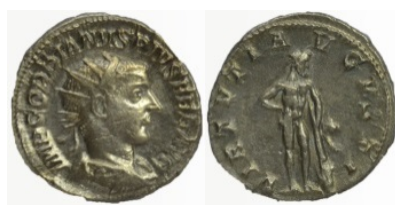

098

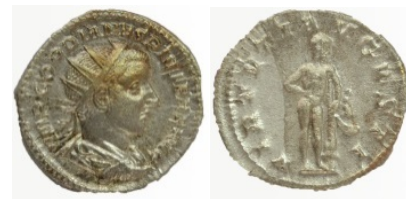

101
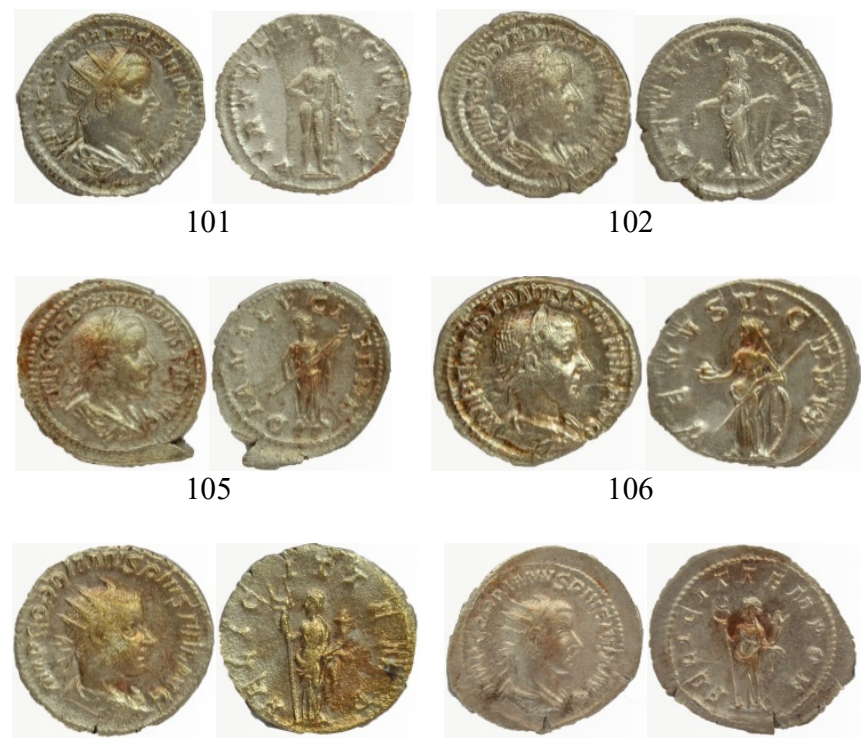

109
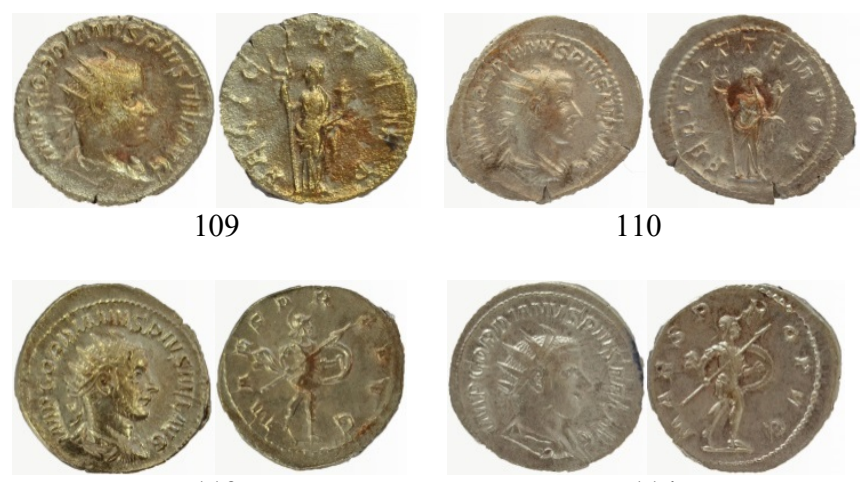

113

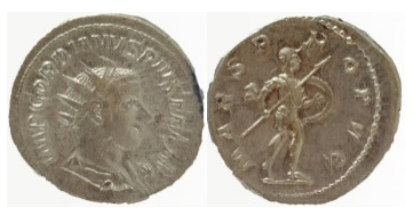

114

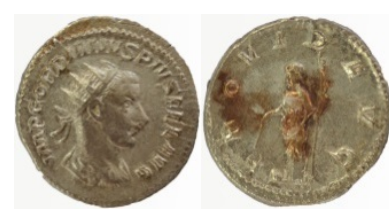

117

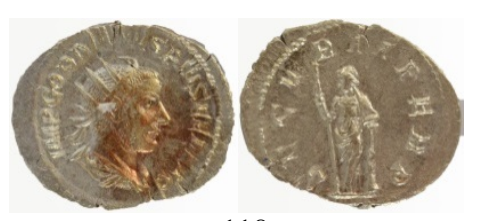

118

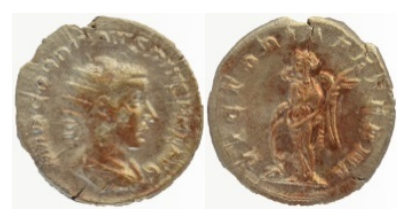

121

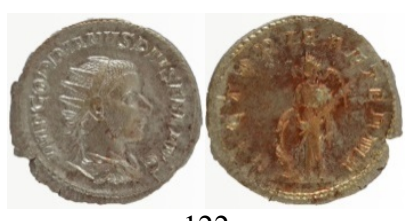

122 


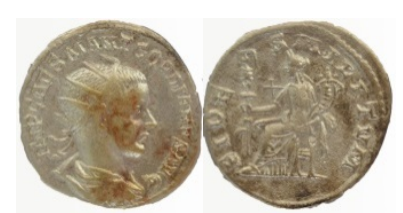

123

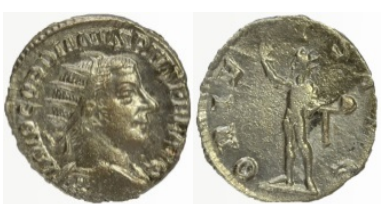

127

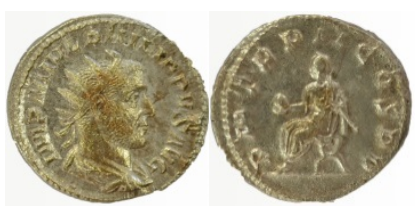

131

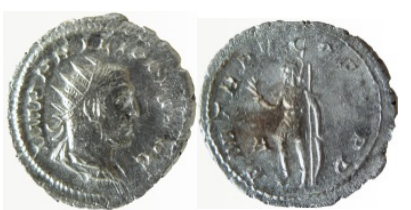

135

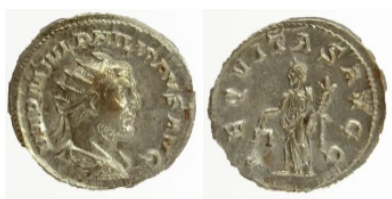

139

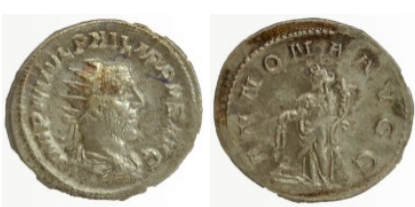

143

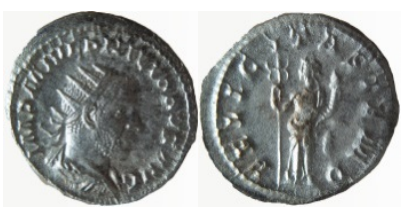

147

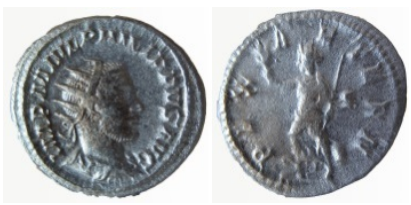

151

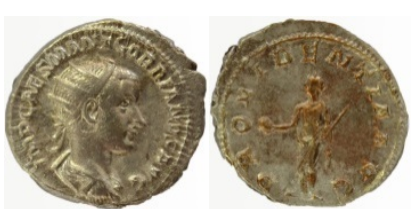

124

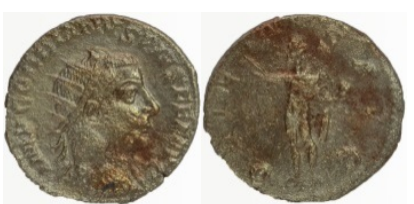

128

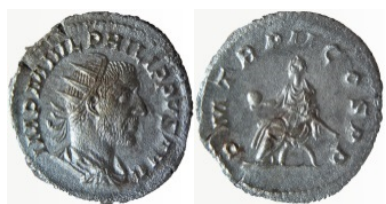

132

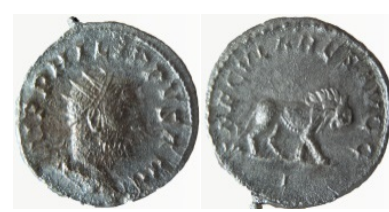

136

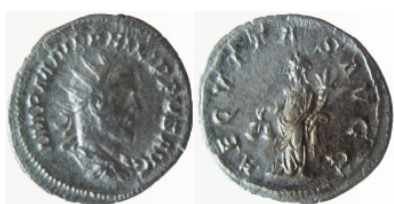

140

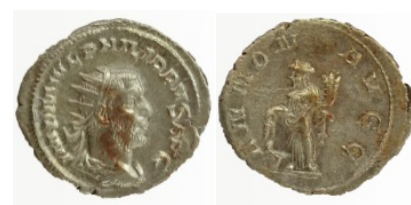

144

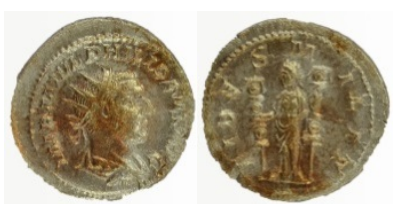

148

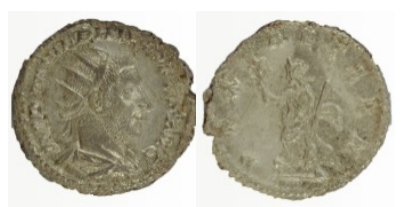

152

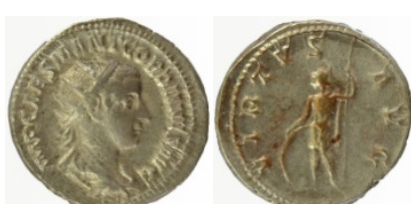

125

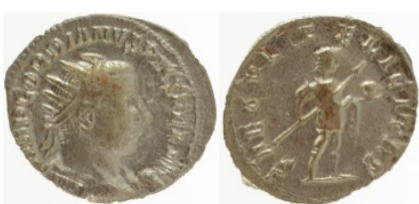

129

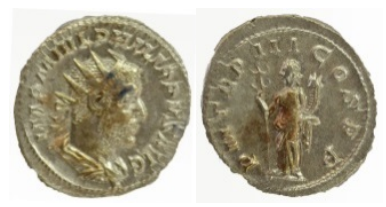

133

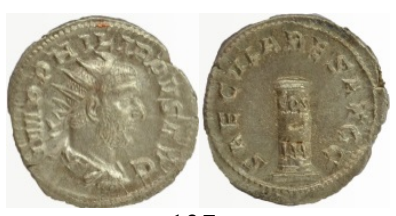

137

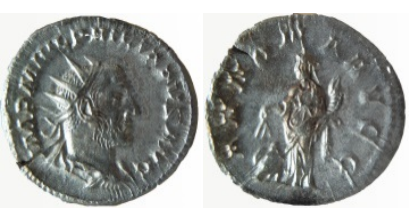

141

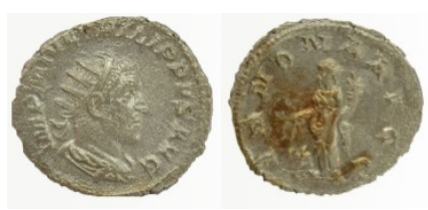

145

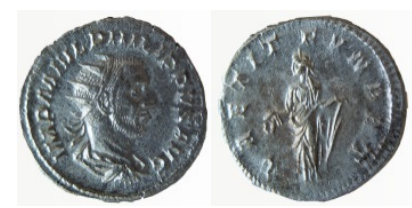

149

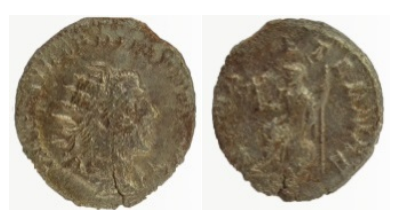

153

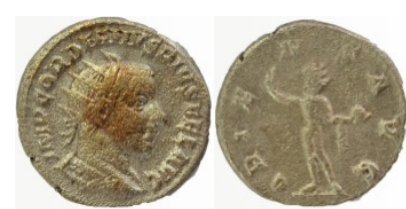

126

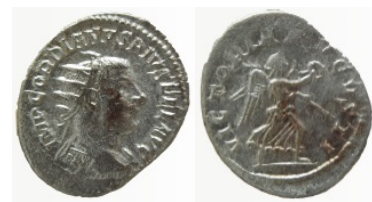

130

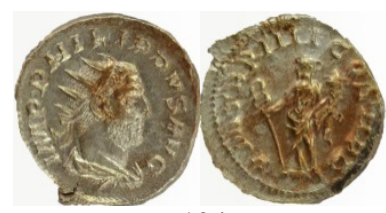

134

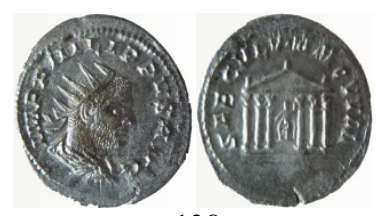

138

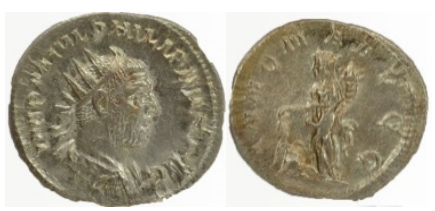

142

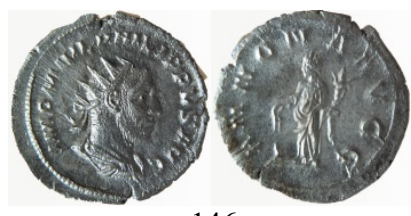

146

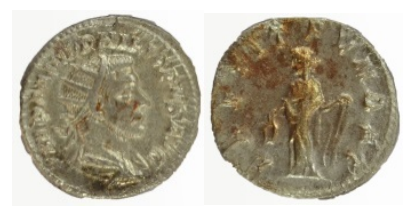

150

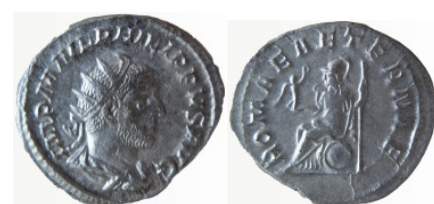

154 


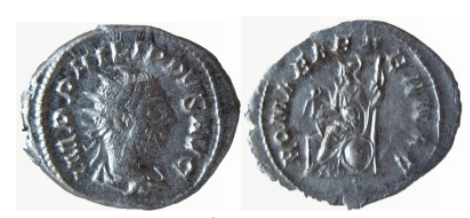

155

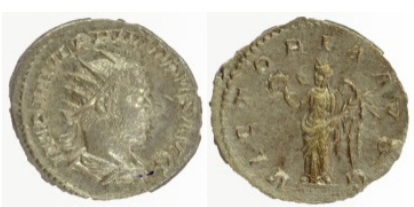

159

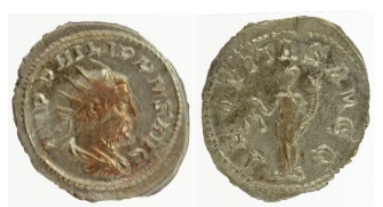

163

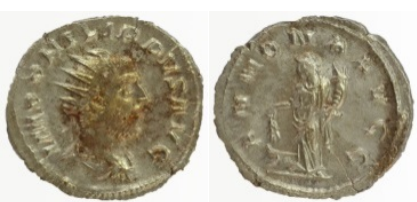

167

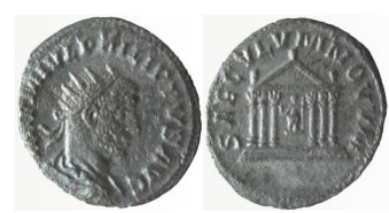

171

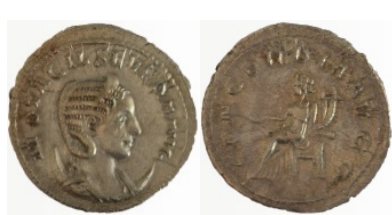

175

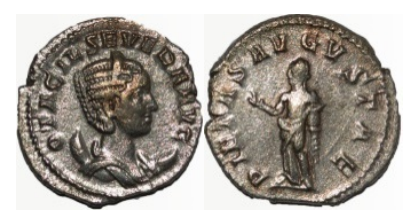

179

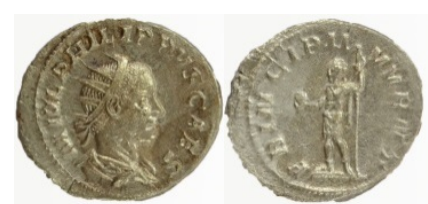

183

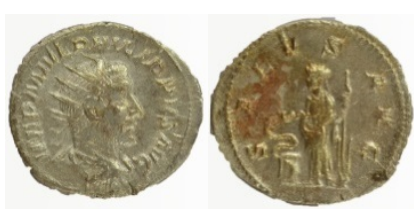

156

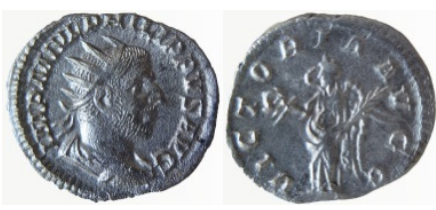

160

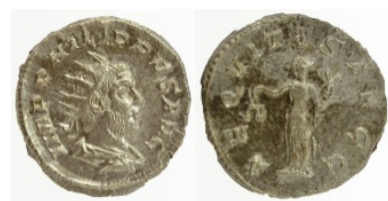

164

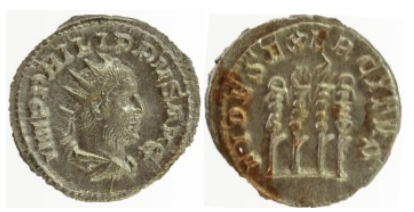

168

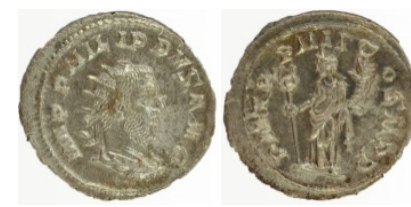

172

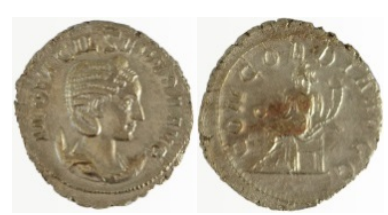

176

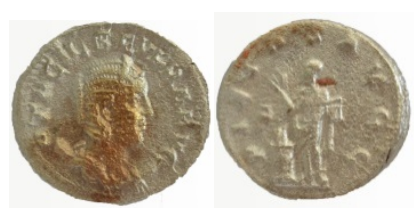

180

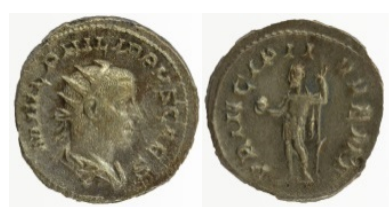

184

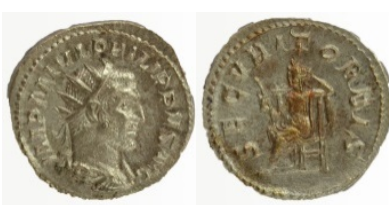

157

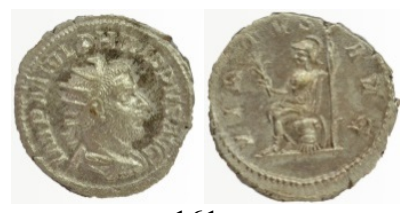

161

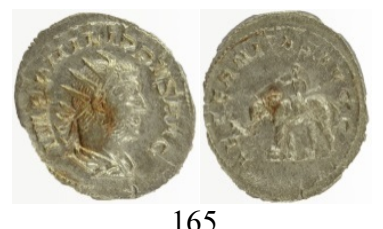

165

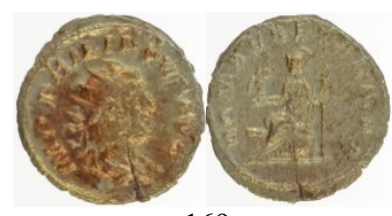

169

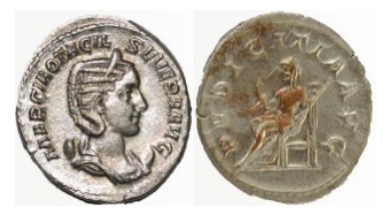

173

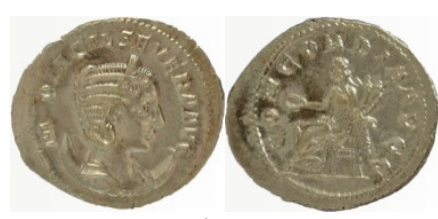

177

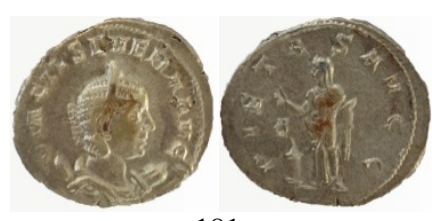

181

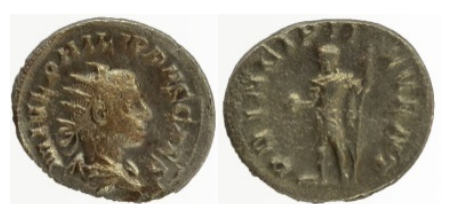

185
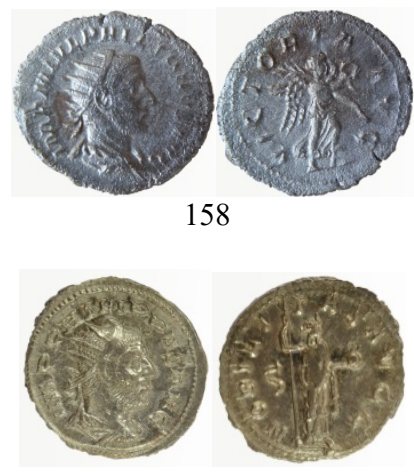

162

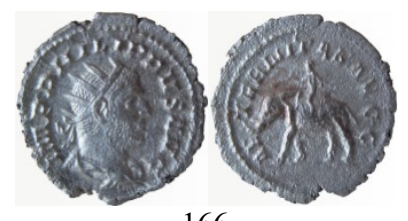

166

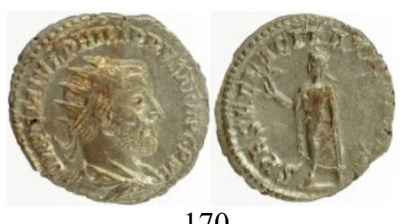

170

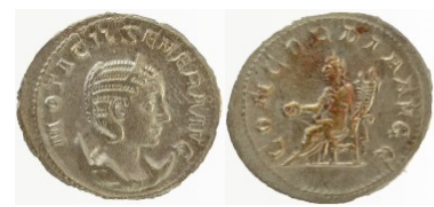

174

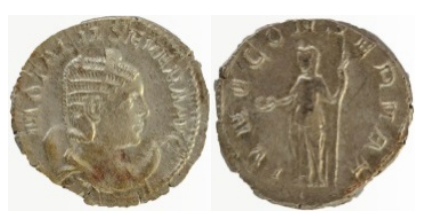

178

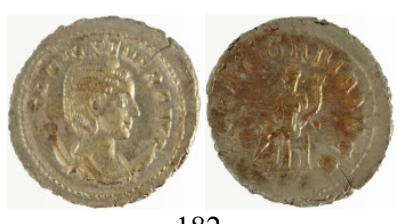

182

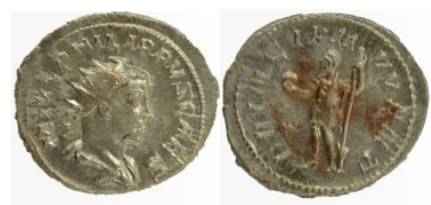

186 


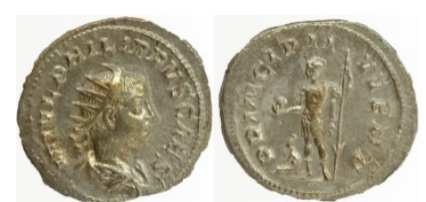

187

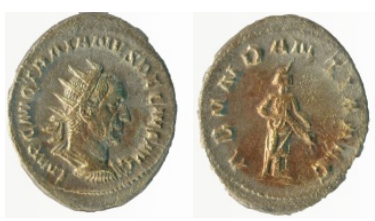

191

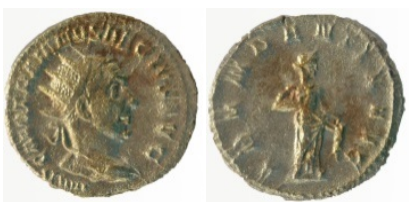

195

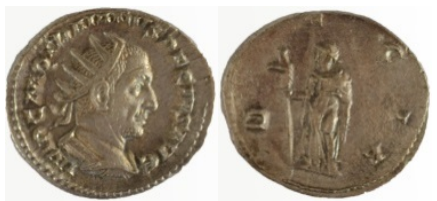

199

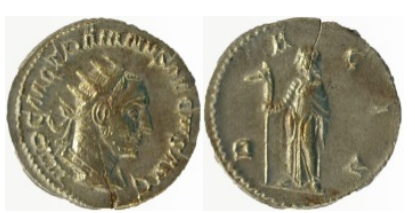

203

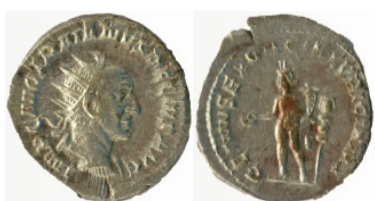

207

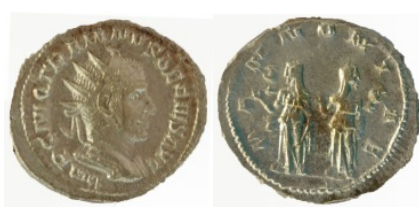

211

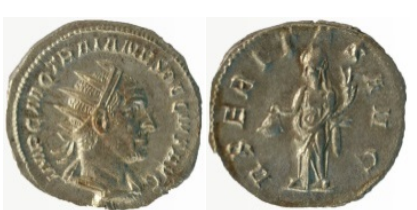

215

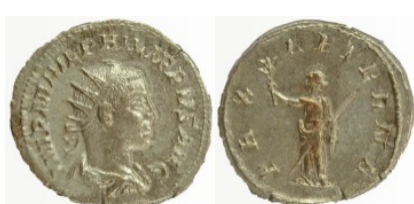

188

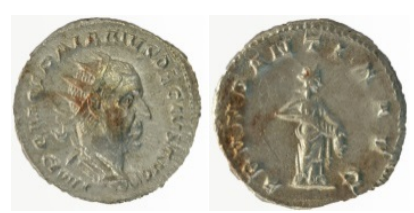

192

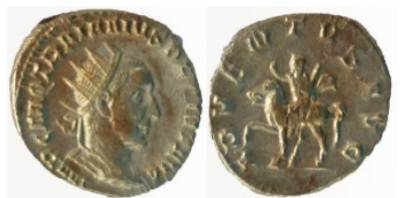

196

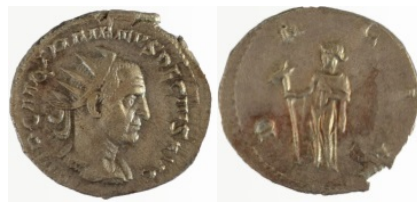

200

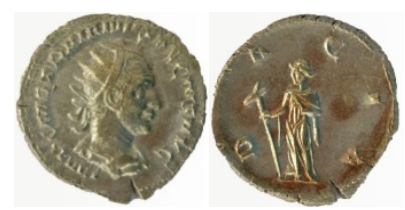

204

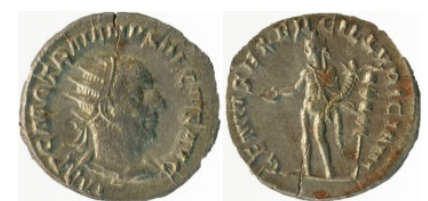

208

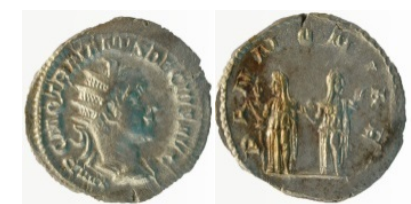

212

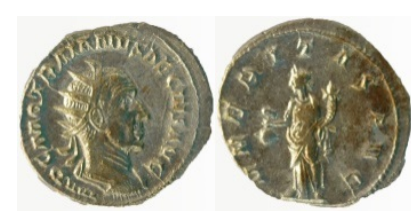

216

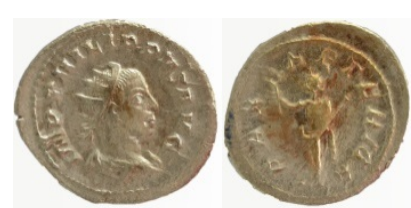

189

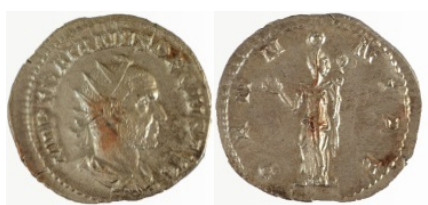

190

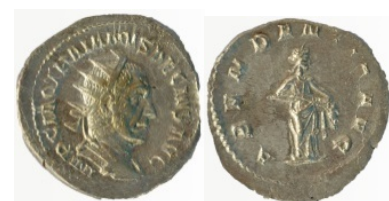

193

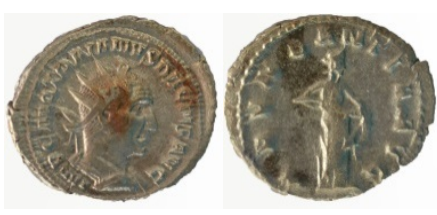

194

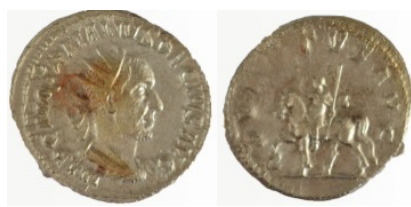

197

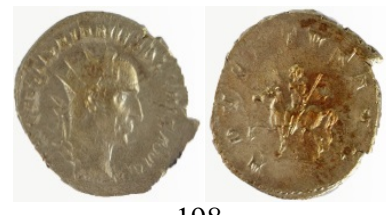

198

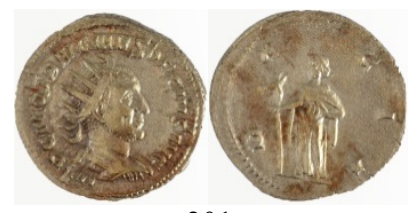

201

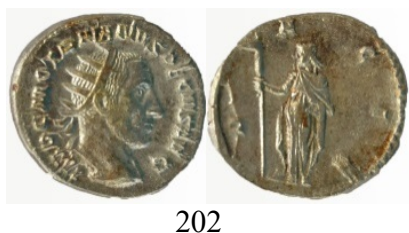

202

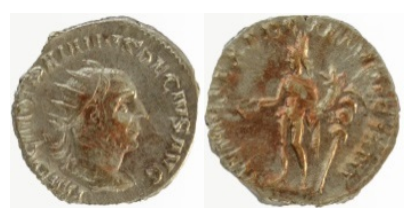

205
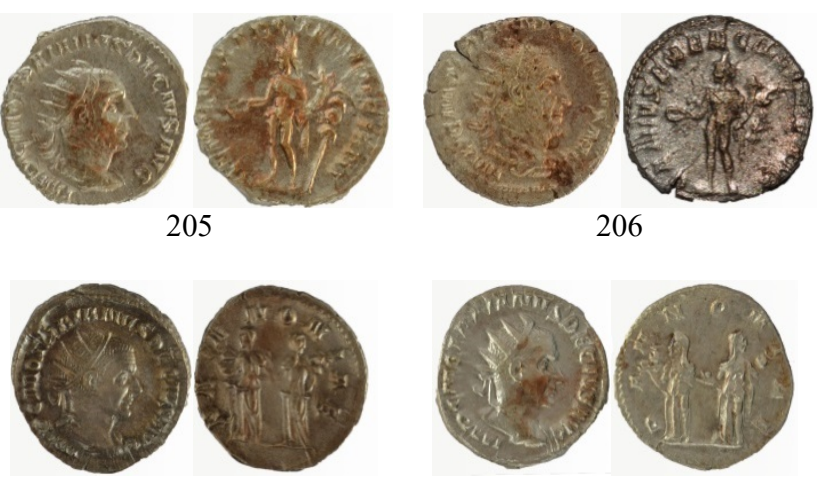

209

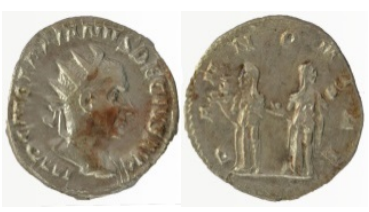

210

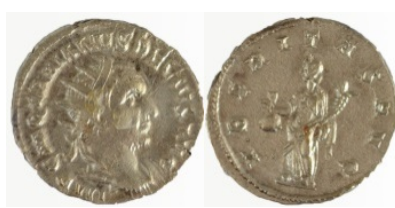

213

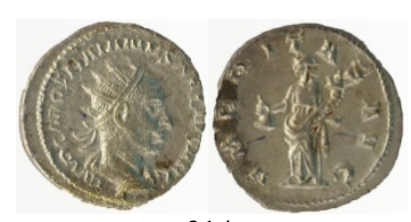

214

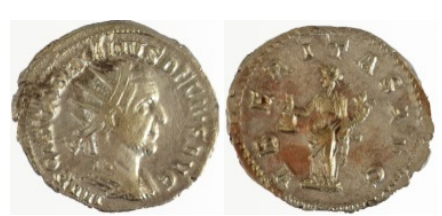

217

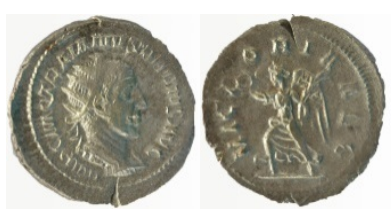

218 


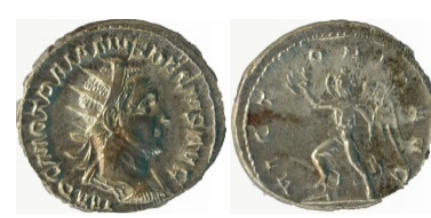

219

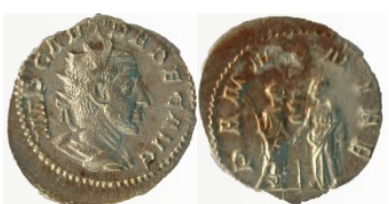

223

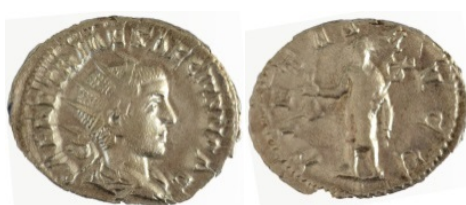

227

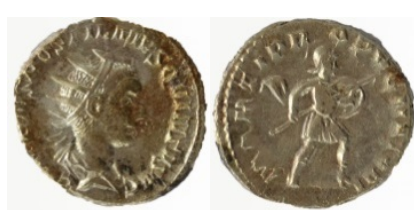

231

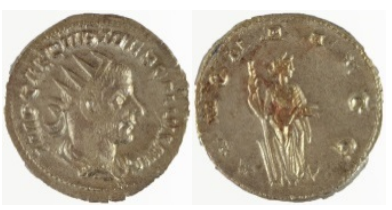

235

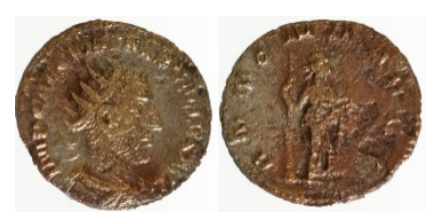

239

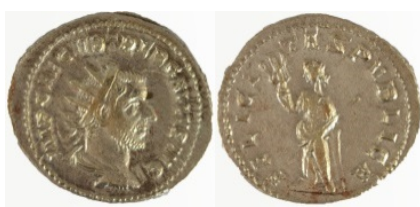

243

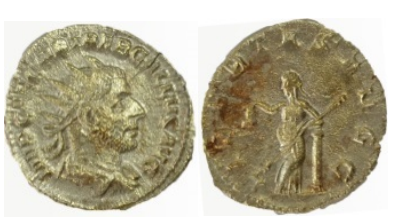

247

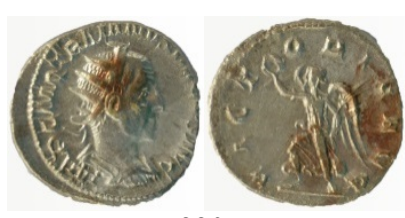

220

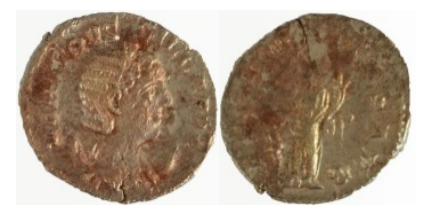

224

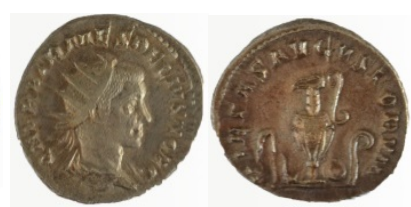

228

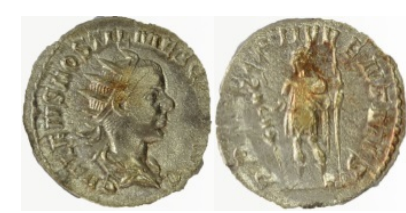

232

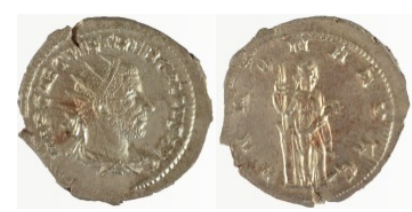

236

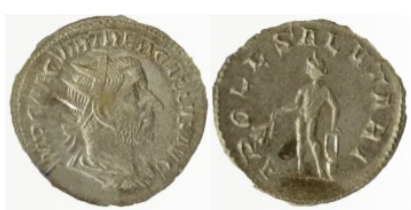

240

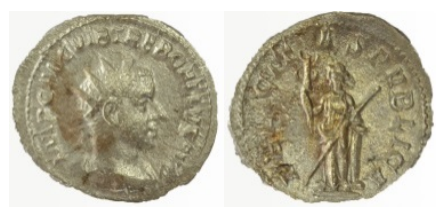

244

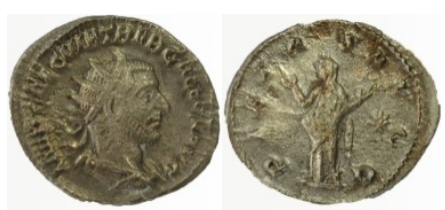

248

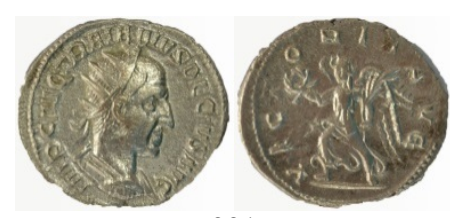

221

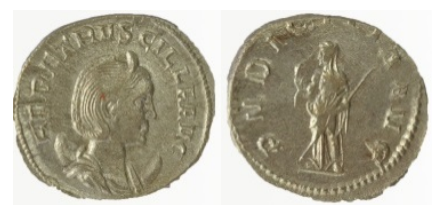

225

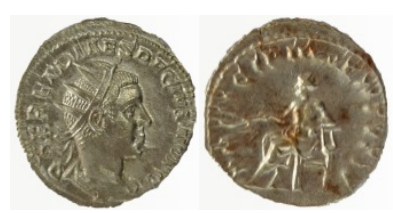

229

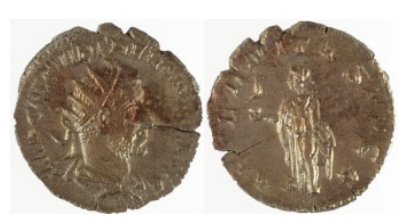

233

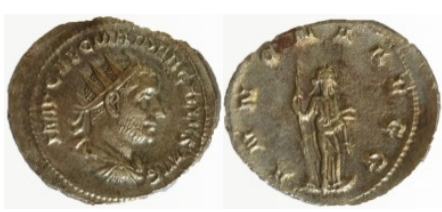

237

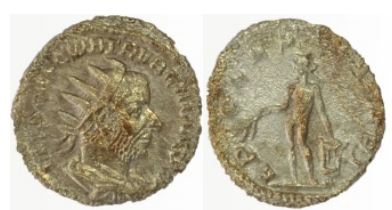

241

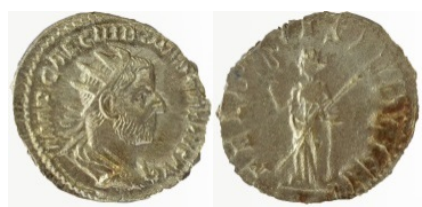

245

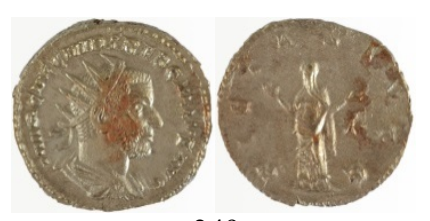

249

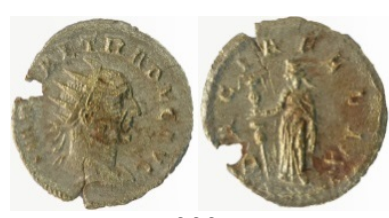

222
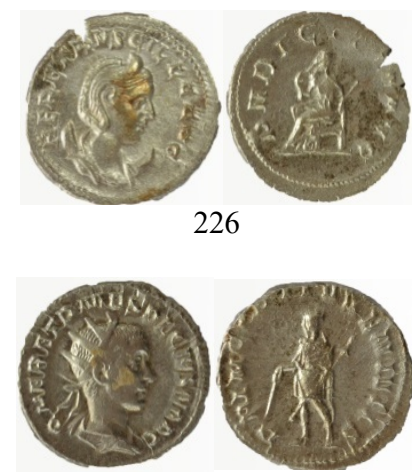

230

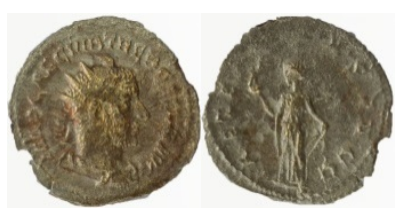

234

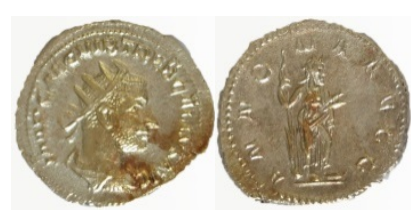

238

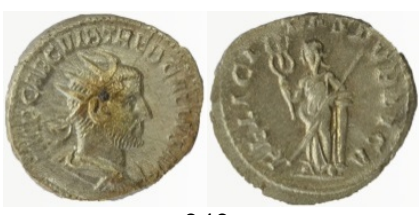

242

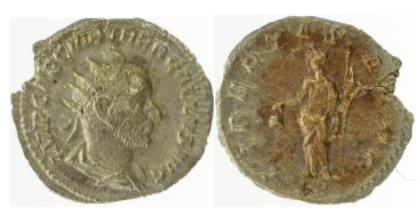

246

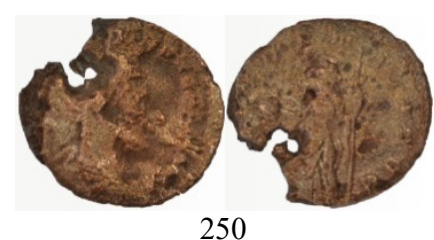




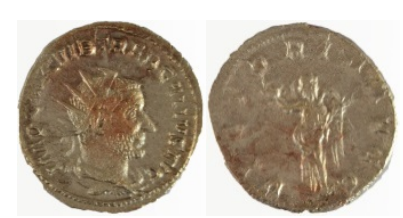

251

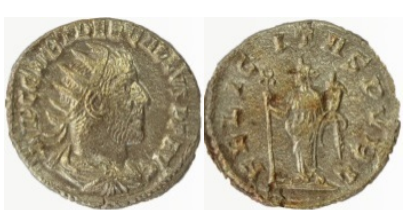

255

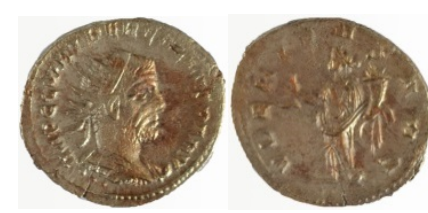

259

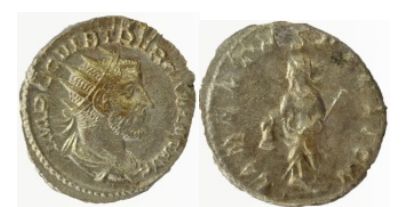

263

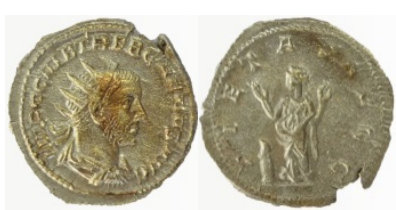

267

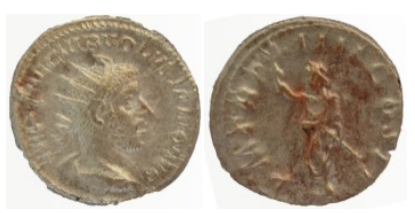

271

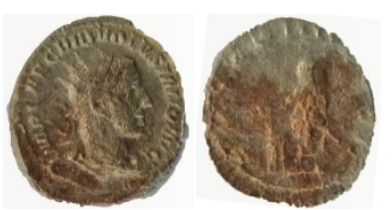

275

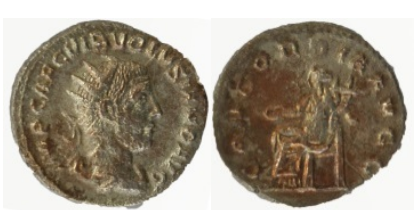

279

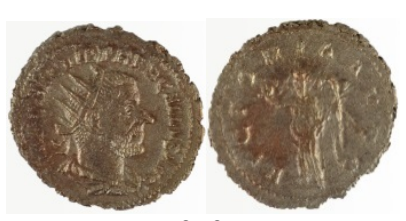

252

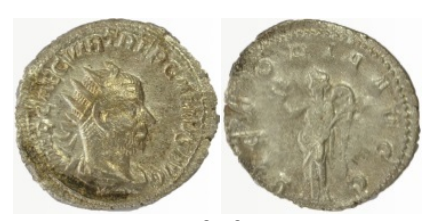

253

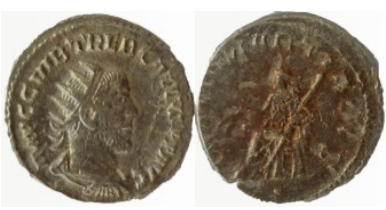

256

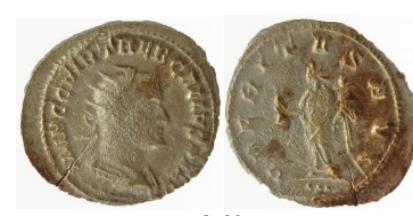

260

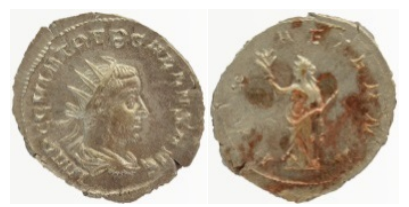

264

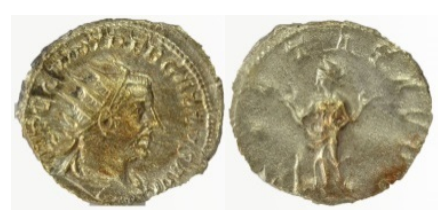

268

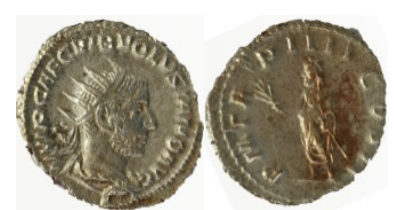

272

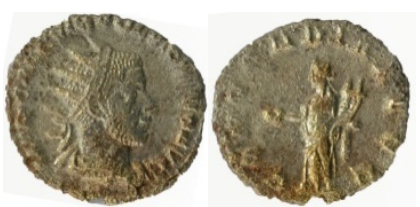

276

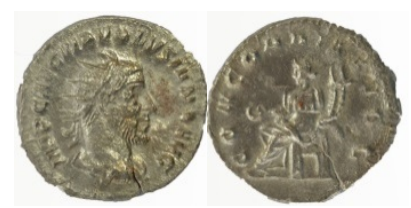

280

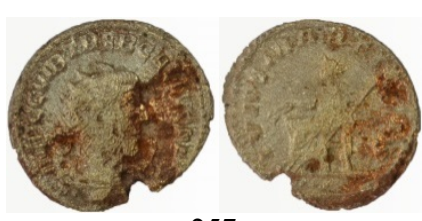

257

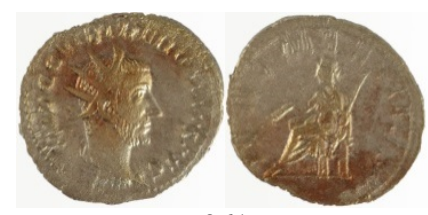

261

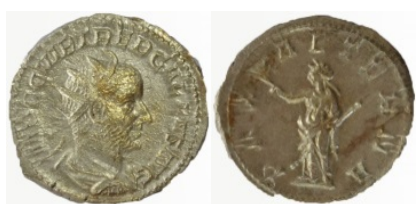

265

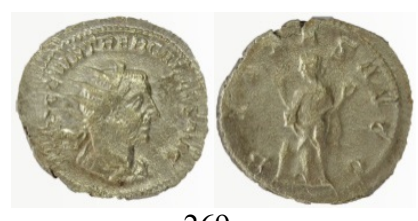

269

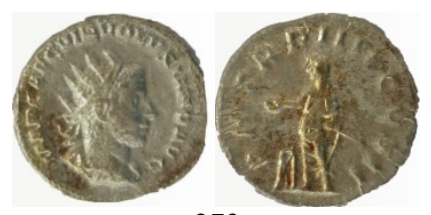

273

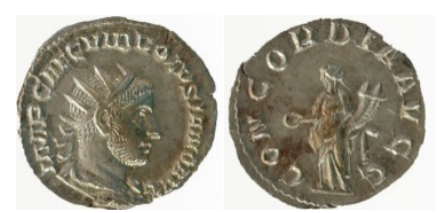

277

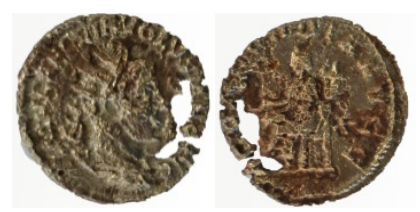

281

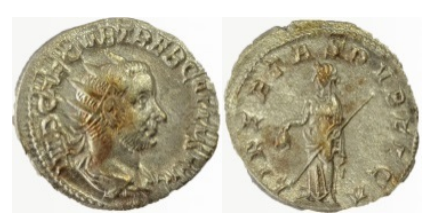

254

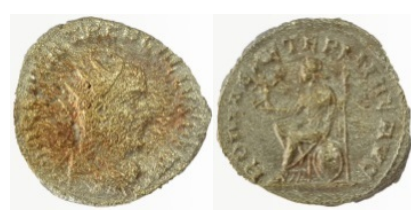

258

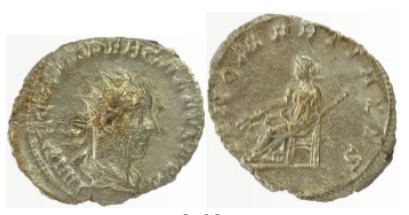

262

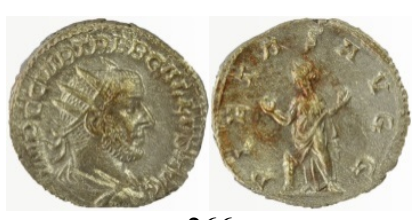

266

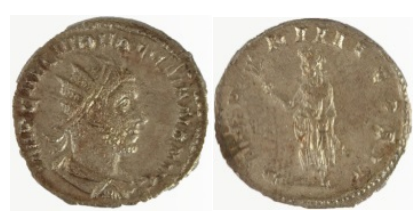

270

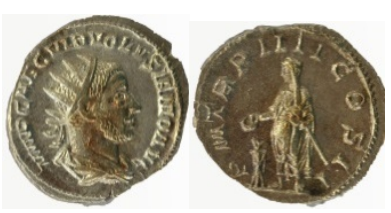

274

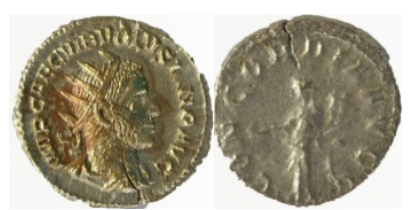

278

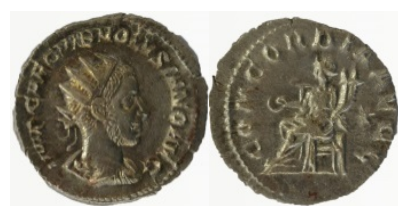

282 


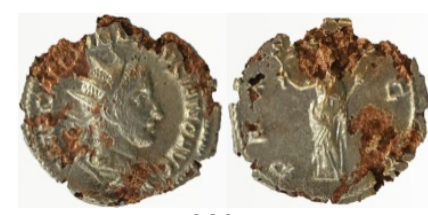

283

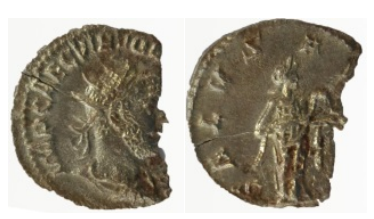

287

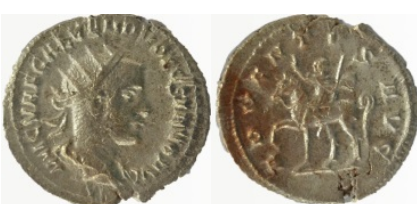

291

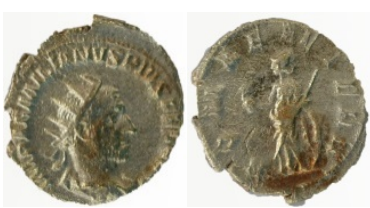

295

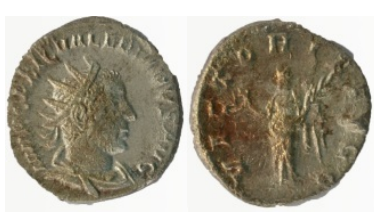

299

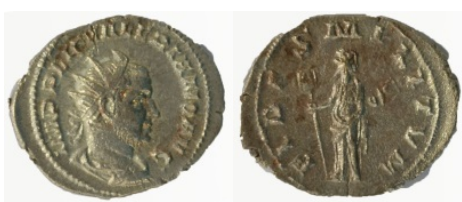

303

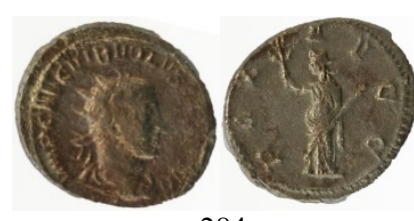

284

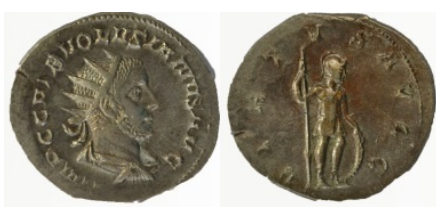

288

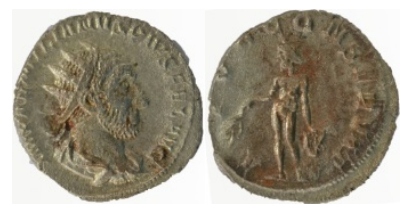

292

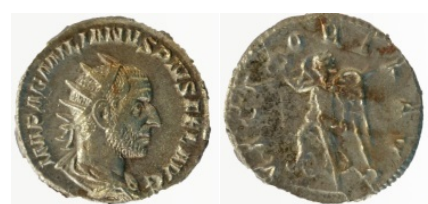

296

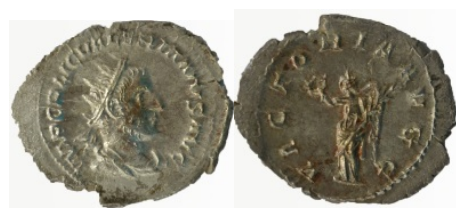

300

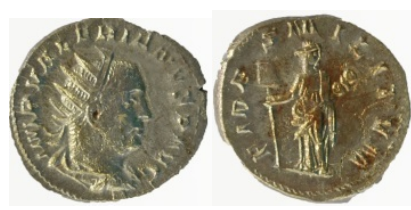

304

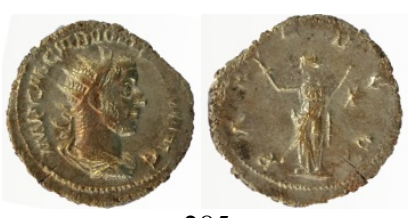

285

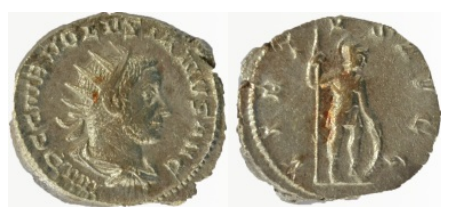

289

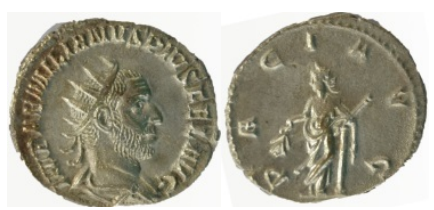

293

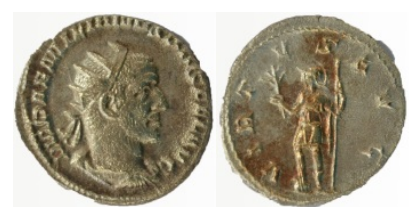

297

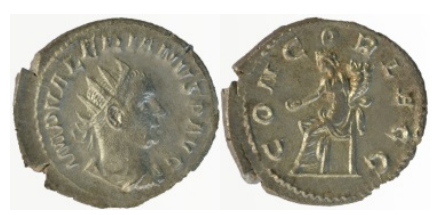

301

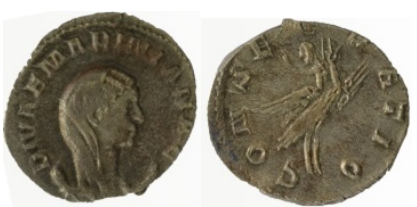

305
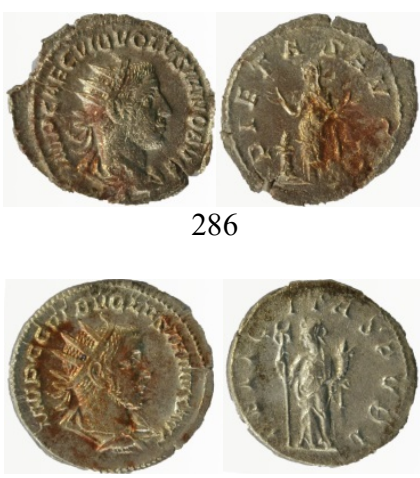

290

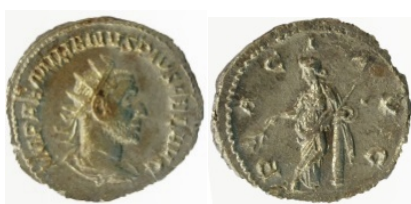

294

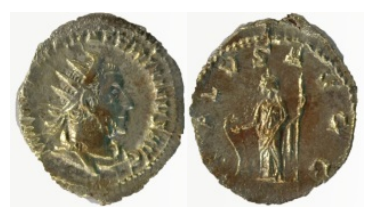

298

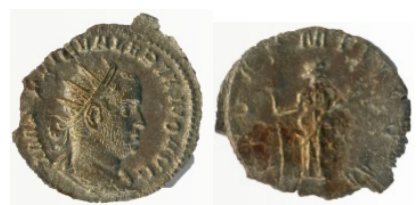

302

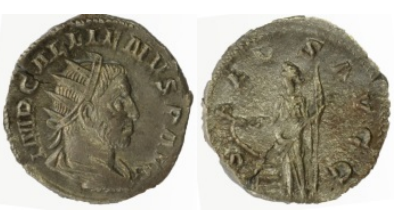

306 
